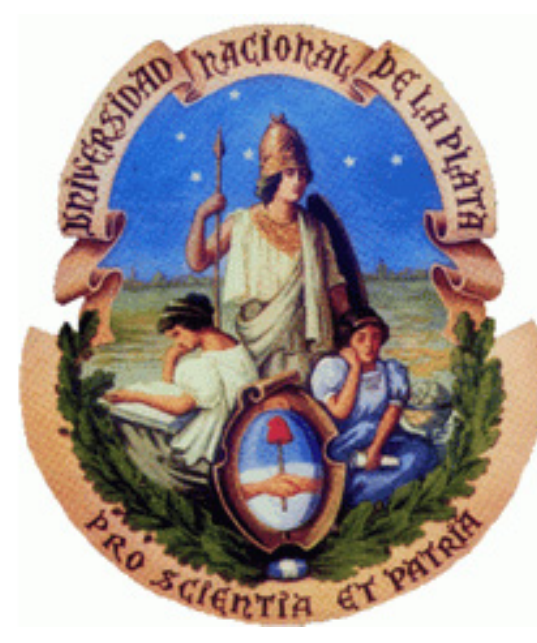

UNIVERSIDAD NACIONAL DE LA PLATA

FACULTAD DE CIENCIAS EXACTAS

DEPARTAMENTO DE FÍSICA

Trabajo de Tesis Doctoral

\title{
Estudio de la influencia del confinamiento en las propiedades críticas de un modelo con tres estados de espín
}

\author{
LiC. LuCiana Melina LuQUe
}

Directores: Dr. Ezequiel Vicente Albano

DR. SANTIAGO ANDRÉS GRIGERA

AÑo 2019 



\section{RESUMEN}

$\mathrm{E}$ 1 objetivo de esta tesis consiste en estudiar los efectos del confinamiento en un modelo con tres estados de espín y, en particular, la segregación del tercer estado de espín, $s_{i}=0$ (que también suele ser identificado como impurezas) en la interfase. Dicho fenómeno se conoce como adsorción interfacial. Para ello se combinarán técnicas computacionales y métodos utilizados en la mecánica estadística. En una primera etapa, estas herramientas permitirán caracterizar el modelo de Blume-Emery-Griffiths (BEG) con interacciones tanto a primeros como a segundos vecinos, mediante la construcción de su diagrama de fases. En este modelo, las impurezas pueden crearse/destruirse en cualquier sitio de la red, por lo que se consideran impurezas móviles. La caracterización de dicho modelo es la base del presente trabajo. Luego, a partir del diagrama de fases del modelo BEG, se estudiarán los efectos causados por el confinamiento sobre este, en particular las transiciones de mojado, las cuales se pueden caracterizar mediante el empleo de una hipótesis de escala recientemente propuesta, y la adsorción interfacial. Por último, se estudiarán los efectos causados por el confinamiento sobre un modelo con desorden congelado (Random Bond Ising Model), es decir, un modelo en el cual las impurezas causadas por la aleatoriedad de la constante de acoplamiento se encuentren fijas. En dicho estudio se observa una violación a la hiper escala, por lo tanto, se propone una nueva hipótesis de escala de tamaño finito que permite caracterizar el RBIM. 



\section{Agradecimientos}

Que lo breve de mis palabras no sea medida de lo agradecida que estoy.

- A mis directores Ezequiel y Santiago, por aceptar la dirección de esta tesis y por permitirme formar parte de su grupo de investigación.

- A mi familia, por ser el origen de mi sistema de referencias. Por su soporte incondicional que me mantiene en pié cada vez que la vida intenta torcerme.

- A Joaquín, por ser mi compañero de vida en estos casi 5 años. Por aguantar, comprender, hablar, callar, escuchar, pero por sobre todas las cosas, por sacar lo mejor de mi.

- A mi familia del IFLySiB por contenerme, ayudarme y enseñarme, tanto en lo académico como en lo personal. Especialmente a Celeste y a Marisa.

- A mis amigas y amigos de acá, de allá, de ahora, de siempre. Gracias por estar incondicionalmente sin importar el tiempo y la distancia.

- A CONICET por el financiamiento.

¡Muchas gracias!! 

For in much wisdom is much grief, and he that increaseth knowledge increaseth sorrow.

[Ecclesiastes 1:18] 



\section{ÍNDICE}

Página

Índice de tablas $\quad$ xi

Índice de figuras $\quad$ xiii

1 Introducción 1

2 Métodos Monte Carlo $\quad 7$

2.1 Monte Carlo y la termodinámica . . . . . . . . . . . . . . . . . . 8

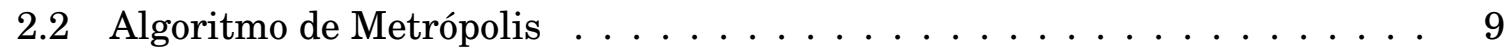

2.3 Algoritmo de Wang-Landau . . . . . . . . . . . . . . . . . . . 12

2.4 WL modificado: Belardinelli-Pereyra . . . . . . . . . . . . . . . . . 16

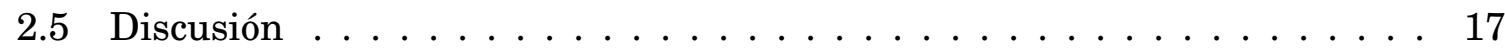

3 Mecánica estadística y Termodinámica 19

3.1 Transiciones de Fase $\ldots \ldots \ldots \ldots \ldots$

3.2 Leyes de Escala . . . . . . . . . . . . . . . . . . . . . . . . . 21

3.2.1 Leyes de Escala para sistemas finitos . . . . . . . . . . . . . . 26

3.3 Determinación de la temperatura crítica en el límite termodinámico $\quad \ldots \quad 29$

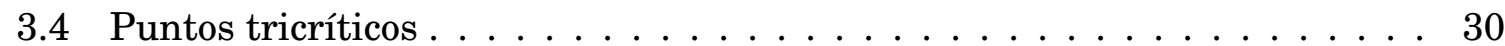

3.4.1 Leyes de escala para los puntos tricríticos . . . . . . . . . . . 32 


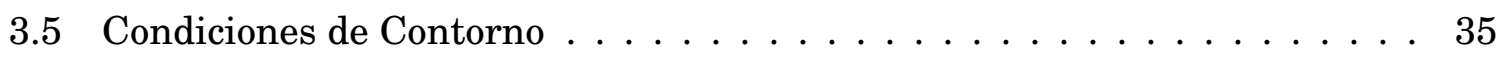

3.5.1 Condiciones Periódicas de Contorno . . . . . . . . . . . . . 35

3.5.2 Condiciones Antiperiódicas de Contorno . . . . . . . . . . . . 36

3.5.3 Condiciones de Contorno Libres . . . . . . . . . . . . . . . 36

4 Modelo BEG $\quad 37$

$4.1 \quad$ El Modelo . . . . . . . . . . . . . . . . . . . . . 38

4.2 Diagrama de fases para $T=0 \ldots \ldots \ldots \ldots \ldots \ldots$

4.3 Diagrama de fases para $T \neq 0 \ldots \ldots \ldots \ldots \ldots$

4.4 Transiciones de primer orden y puntos tricríticos $\ldots \ldots \ldots \ldots$. . . . . . 44

4.5 Fase Cuadrupolar . . . . . . . . . . . . . . . . . . 50

4.6 Modelo de BEG con interacción a segundos vecinos . . . . . . . . . . . . 54

4.6.1 Diagrama de fases para $T=0 \ldots \ldots \ldots \ldots$. . . . . . . 54

4.6.2 Diagrama de fases para $T \neq 0 \ldots \ldots \ldots \ldots$

5 Estudio del efecto del confinamiento en el modelo BEG 59

5.1 El modelo . . . . . . . . . . . . . . . . . . . . 61

5.2 Wetting crítico en dos dimensiones . . . . . . . . . . . . 63

5.2.1 Resultados Monte Carlo . . . . . . . . . . . . . . . . . 70

5.3 Adsorción Interfacial $\ldots \ldots \ldots \ldots \ldots \ldots$

5.4 Integración Termodinámica $\ldots \ldots \ldots \ldots \ldots$

6 Estudio del confinamiento en el modelo RBIM 87

$6.1 \quad$ El Modelo . . . . . . . . . . . . . . . . . . . . . . 88

6.2 Violación de la hiper escala $\ldots \ldots \ldots \ldots \ldots \ldots$

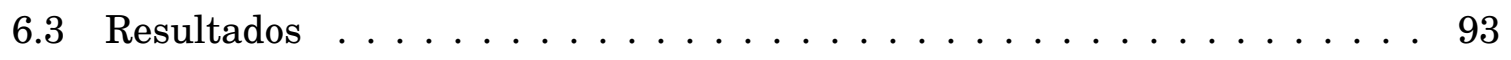

6.3.1 Detalles sobre las simulaciones . . . . . . . . . . . 93

6.3.2 Resultados de las simulaciones . . . . . . . . . . . . . . . 94 
$\begin{array}{lll}7 & \text { Conclusiones } & 105\end{array}$

A Obtención del parámetro de orden para el punto tricrítico 109

B Modelo BEG: Obtención del hamiltoniano $\quad 115$

$\begin{array}{ll}\text { Bibliografía } & 123\end{array}$ 



\section{ÍNDICE DE TABLAS}

TABLA

Página

4.1 Puntos tricríticos para $\alpha=0 \ldots \ldots \ldots \ldots \ldots \ldots$

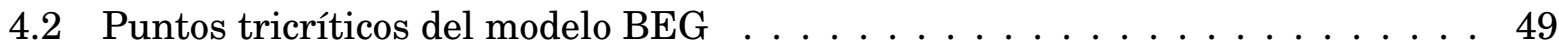

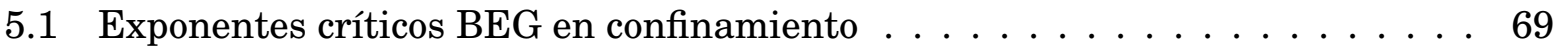





\section{ÍNDICE DE FIGURAS}

FIGURA

Página

2.1 Diagrama de flujo: Algoritmo de Metrópolis. . . . . . . . . . . . . . . . 10

2.2 Diagrama de flujo: Algoritmo de Wang-Landau . . . . . . . . . . . . . . . 13

3.1 Límite termodinámico vs tamaño finito . . . . . . . . . . . . . . . . . 29

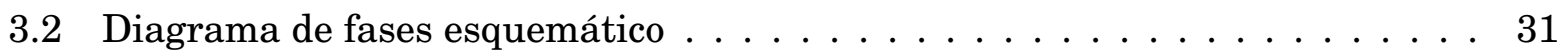

3.3 Diagramas de fases simétrico y antisimétrico . . . . . . . . . . . . . 32

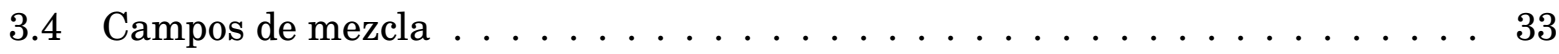

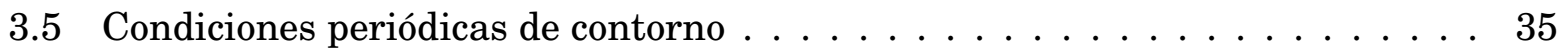

4.1 Diagrama de fases del modelo BEG para $T=0 \ldots \ldots \ldots \ldots$

4.2 Diagrama de fases del modelo BEG . . . . . . . . . . . . . . . . . 41

4.3 Magnetización y Cumulante . . . . . . . . . . . . . . . . . . 43

4.4 Transiciones de primer y segundo orden . . . . . . . . . . . . . . 45

4.5 Transiciones de primer orden $\ldots \ldots \ldots \ldots \ldots$

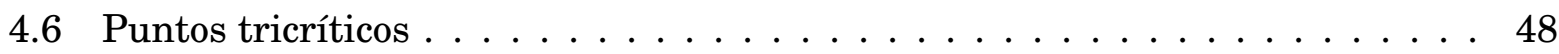

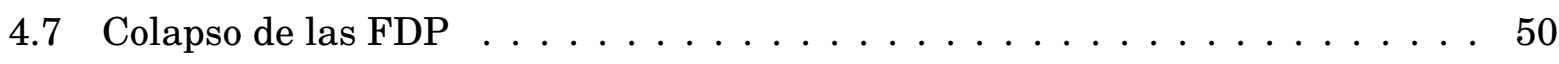

4.8 Fase Cuadrupolar $\ldots \ldots \ldots \ldots \ldots \ldots \ldots \ldots \ldots \ldots$

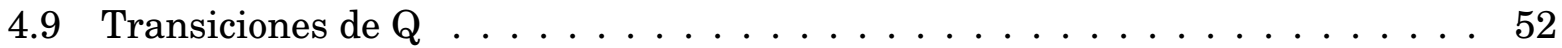

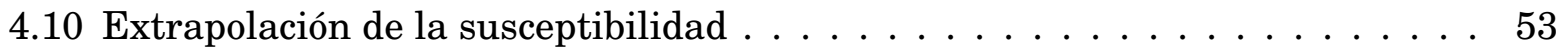

xiii 
4.11 Diagrama de fases para $T=0$ del modelo BEG con interacción NNN . . . . 56

4.12 Diagrama de Fases del modelo BEG con interacción NNN . . . . . . . . . . 57

4.13 Transición $Q \rightarrow Q_{F} \ldots \ldots \ldots \ldots \ldots \ldots \ldots \ldots$

5.1 Transiciones de Mojado y Secado . . . . . . . . . . . . . . . . . . . 60

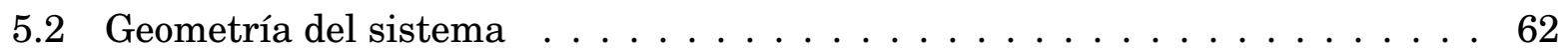

5.3 Transiciones de Mojado . . . . . . . . . . . . . . . . . . . 71

5.4 Snapshots . . . . . . . . . . . . . . . . . . . 72

5.5 Susceptibilidad . . . . . . . . . . . . . . . 73

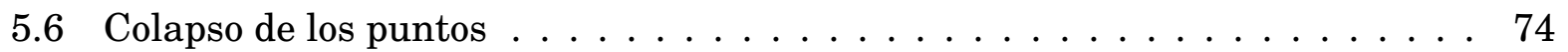

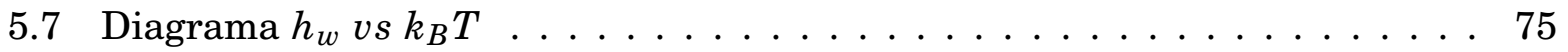

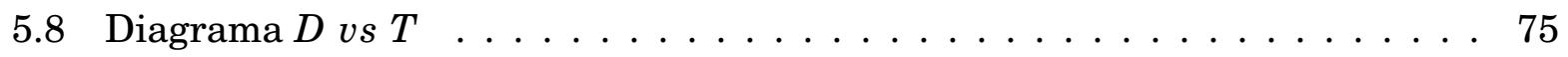

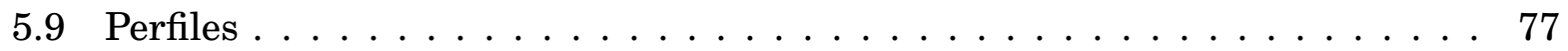

5.10 Densidad de Vacancias . . . . . . . . . . . . . . . . . . 78

5.11 Adsorción Interfacial $\ldots \ldots \ldots \ldots \ldots \ldots \ldots$

5.12 Adsorción Interfacial en función de $\alpha \ldots \ldots \ldots \ldots$. . . . . . 80

5.13 Integración termodinámica $D=-\infty \ldots \ldots \ldots \ldots \ldots$

5.14 Energía libre interfacial $\ldots \ldots \ldots \ldots \ldots \ldots \ldots$

5.15 Integración Termodinámica $D \neq-\infty \ldots \ldots \ldots \ldots \ldots$

6.1 Magnetización para $T=1,75$ en el RBIM . . . . . . . . . . . . . . 95

6.2 Magnetización al cuadrado para $T=1,75$ en el RBIM . . . . . . . . . . . . 96

6.3 Susceptibilidad y Cumulante para $T=1,75$ en el RBIM $\ldots \ldots \ldots$. . . . . 97

6.4 Extrapolación de la susceptibilidad en el RBIM para $T=1,75 \ldots \ldots$. . . . . 98

6.5 Magnetización para $T=1,5$ en el RBIM . . . . . . . . . . . . . . . 100

6.6 Magnetización al cuadrado para $T=1,5$ en el RBIM _ . . . . . . . . . 101

6.7 Susceptibilidad y Cumulante para $T=1,5$ en el RBIM . . . . . . . . . . . 102

6.8 Extrapolación de la susceptibilidad en el RBIM para $T=1,5 \ldots 103$ 


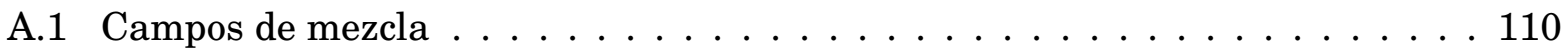

B.1 Diagramas de Fases $H e^{3}$ y $H e^{4} \ldots \ldots \ldots \ldots \ldots \ldots$

B.2 Diagramas de Fases $H e^{3}$. Super fluido . . . . . . . . . . . . . . 117 



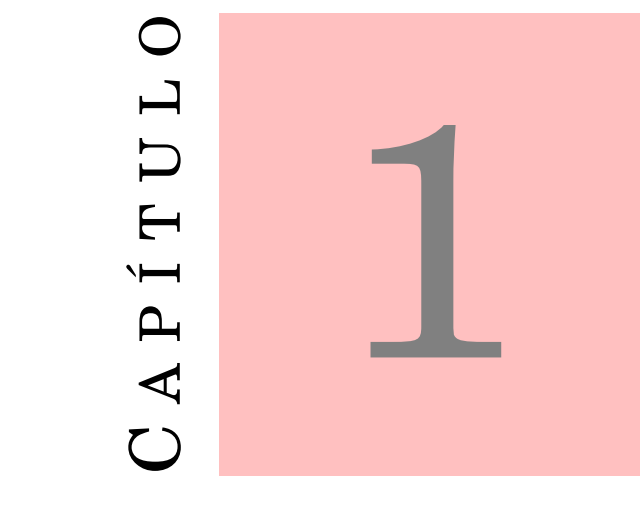

INTRODUCCIÓN

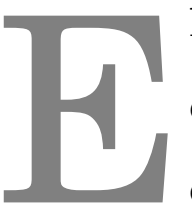

1 estudio y comprensión de las transiciones de fase y los fenómenos críticos que ocurren en geometrías confinadas son tópicos de gran interés tanto a nivel científico como tecnológico. La combinación de efectos de borde y confinamiento por tamaño finito, ambos debido a la interacción del sistema físico con las paredes que lo contienen, modifican la fenomenología respecto de la que ocurre en el límite termodinámico [1-3]. En efecto, la criticidad no se manifiesta como divergencias en los observables o sus momentos sino como picos suaves que, además, están desplazados respecto de las divergencias del límite termodinámico $[4,5]$. Este corrimiento se debe fundamentalmente a los efectos de superficie, mientras que la suavidad que adquieren los picos está relacionada con los efectos de tamaño finito [6]. Estos fenómenos se han vuelto de fundamental interés al plantearlos como un problema de la mecánica estadística. En esta tesis se estudiarán sistemas en el marco de modelos de red con geometrías 
confinadas, y con interacciones a primeros y segundos vecinos, sometidos a fuerzas de corto alcance causadas por las paredes.

Una gran cantidad de trabajos se han centrado en el estudio de modelos de Ising simples con campos superficiales; ver por ejemplo [7] para una revisión y [4, 8-26] para trabajos originales. La simetría de éste modelo implica que la coexistencia de fases entre las fases ordenadas con magnetizaciones espontáneas positiva y negativa ocurren para campo de bulk $h_{b}=0$ en el límite termodinámico. Lo mismo ocurre si se estudia un film de ancho finito $L$ con superficies sobre las cuales actúan campos superficiales $h_{1}$ y $h_{L}$. Si se considera la situación anti simétrica $h_{1}=-h_{L}$, al incrementar la temperatura, la interfase que separa las fases ordenadas se alejará de la pared y se moverá libremente en el centro del film. Este fenómeno se puede relacionar con las transiciones de mojado (wetting en inglés). Por otro lado, si $h_{1}=h_{L}$, es posible relacionar este fenómeno con la condensación capilar $[9,11]$, la cual consiste en un corrimiento en el valor del campo de bulk $\left(h_{b} \neq 0\right)$ debido a los efectos de borde, para el cual ocurrirá la coexistencia de fases en el bulk.

Particularmente, un área de estudio desafiante, es la de los efectos del confinamiento cuando se agregan impurezas no magnéticas. Esto produce un cambio en las transiciones del bulk y, por lo tanto, en los fenómenos causados por el confinamiento. Trabajos de este estilo se han llevado a cabo previamente por Albano y Binder [27], donde los autores proponen una teoría de escala que permite describir los resultados de las simulaciones computacionales del wetting crítico, haciendo uso de un nuevo parámetro de orden con exponente crítico $\beta=0$. Dichos estudios se realizan utilizando el modelo de Blume-Capel (BC) [28, 29]. Este modelo es una extensión del modelo de Ising, en el que cada sitio de la red $i$ se puede encontrar en tres estados distintos, $1,-1$ y 0 y el Hamiltoniano tiene la forma

$$
\mathscr{H}=-J \sum_{\langle i, j\rangle} s_{i} s_{j}+D \sum_{i} s_{i}^{2}
$$


donde el campo cristalino $D$ controla la densidad de vacancias, es decir, sitios con $s_{i}=0$. Cuando $D \rightarrow-\infty$, las vacancias son excluidas y se recupera el modelo de Ising estándar, pero cuando $\frac{D}{J}$ es del orden de la unidad, el modelo presenta un diagrama de fases no trivial con un punto tricrítico en $D=D_{t}$. Para $D>D_{t}$ la transición en $T_{c}(D)$ es de primer orden, es decir, coexisten dos fases con magnetizaciones opuestas distintas de cero y una fase desordenada (con magnetización 0).

Uno de los modelos que se utilizará en esta tesis para estudiar el confinamiento, es una extensión del modelo BC llamado Blume-Emery-Griffiths (BEG) [30]. Este fue propuesto en 1971 para estudiar algunas características del comportamiento crítico de la mezcla de $H e^{3}$ y $H e^{4}$, como la separación de fases y la superfluidéz. Al igual que su predecesor, el modelo de BEG también contiene un término de interacción de espines, tipo Ising, donde la variable de espín, $s_{i}$ puede tomar los valores $1,-1$ y 0 , y un término que controla la densidad de vacancias mediante el campo cristalino $D$. Pero además presenta un último término, el cual marca la diferencia entre ambos modelos, encargado de controlar la interacción bicuadrática mediante la constante $\alpha$. Esta favorece la atracción o repulsión de espines del mismo tipo, generando nuevas formas de ordenamiento en el sistema.

Sobre la base de estudios del modelo BC, es bien sabido que las vacancias tienden a segregarse a lo largo de la interfaz entre dominios con diferentes orientaciones de los espines [27, 31]. Dado que el modelo BC corresponde al caso en el que la constante de interacción bicuadrática $\alpha=0$, el estudio del enriquecimiento de vacancias en la interfase, conocido también como adsorción interfacial, es un fenómeno interesante dentro del marco del modelo BEG, por ejemplo para estudiar fenómenos de mojado. De esta manera se puede ver que, al variar el parámetro que regula la interacción entre las vacancias $\alpha$, el modelo BEG muestra un comportamiento de mojado interesante. Este fenómeno ha sido estudiado previamente para el modelo BC [32], pero no ha sido abordado para el 
modelo BEG. Es por eso que el estudio de la adsorción interfacial en el modelo BEG es uno de los objetivos de este trabajo.

Desde que el modelo BEG vio la luz, se ha dedicado mucho trabajo para estudiar su comportamiento y aplicaciones a campos relacionados tales como fluidos ternarios [33, 34], cambios de fases en microemulsiones [35], aleaciones semiconductoras [36], modelos de conducción eléctrica [37], etc. Para ello se han usado diversas técnicas y métodos, tales como aproximaciones de campo medio [30], desarrollos de series de altas temperaturas [38], simulaciones Monte Carlo [39], grupo de renormalización [40, 41], método de variación de cluster [42], teorías de campo efectivo [43-45], etc. En particular en esta tesis los estudios se harán mediante el uso de los algoritmos de Metrópolis [46] y Wang-Landau [47, 48] en su implementación propuesta por Belardinelli-Pereyra [49]. De hecho, al igual que el modelo BC, el modelo BEG presenta comportamiento tricrítico, por lo que el algoritmo de WL puede ser usado para determinar la función de distribución de la probabilidad de los campos de escala y sus variables conjugadas, las cuales permitirán evaluar los puntos tricríticos y realizar análisis de escala tricrítico.

En el modelo BEG, el tercer estado de espín $\left(s_{i}=0\right)$, también identificado como vacancias, provoca desorden dinámico ya que éstas se mueven libremente en la red, es decir, se pueden crear/destruir en cualquier sitio de la misma. Sin embargo, existe otro tipo de desorden el cual se genera cuando el sistema presenta impurezas fijas en sitios aleatorios de la red, este se conoce como desorden congelado (quenched disorder, en inglés). Un ejemplo genérico de como actúa este tipo de desorden se puede ver en el comportamiento de la interfaz entre fases coexistentes con orientación opuesta de la magnetización espontánea en un modelo de Ising [50]. En un sistema "puro", tal interfaz es un objeto crítico, que exhibe fluctuaciones de tipo de onda capilar de longitud de onda larga, la cual gana mucha entropía realizando excursiones locales relativas a una línea de referencia $(d=2)$. La presencia de desorden congelado aleatorio en el sistema 
afecta fuertemente este comportamiento. La competencia aleatoria que existe entre los signos de las constantes de intercambio $J_{i j}$ y los espines ubicados en los sitios vecinos de la red $(i, j)$ puede incluso destruir el orden ferromagnético. El modelo de Ising, en presencia de desorden congelado debido a la constante de acoplamiento, se conoce como modelo de Ising con enlaces aleatorios (RBIM por sus siglas en inglés). Por lo tanto, mientras que para el sistema puro, la interfase recta es claramente el único mínimo de la energía libre, en el sistema con desorden congelado la interfase será rugosa, por lo que el paisaje de la energía libre también lo será. Esto generará configuraciones de interfases rugosas equivalentes que compiten entre sí. Este comportamiento tiene implicaciones importantes para los fenómenos de mojado $[51,52]$ ya que este se puede ver como una transición continua de la interfase desde la pared hasta el centro de la red, y claramente la rugosidad interfacial que se ve incrementada por el desorden congelado aleatorio afectará este comportamiento. Entender las consecuencias de este tipo de desorden fue un problema desafiante durante muchas décadas $[53,54]$ y los conceptos asociados para lidiar con la energía libre, tuvieron y tienen un gran impacto sobre distintos campos como la ciencia computacional, macromoléculas biológicas, etc.

De todo lo expuesto anteriormente, se desprende el objetivo general de esta tesis, el cual consiste en estudiar los efectos del confinamiento en un modelo con tres estados de espín y, en particular, la segregación de vacancias en la interfase. Para ello se combinarán técnicas computacionales y métodos utilizados en la mecánica estadística desarrollados en los capítulos (2) y (3), respectivamente. Estas herramientas permitirán caracterizar el modelo de BEG con interacciones tanto a primeros como a segundos vecinos, mediante la construcción de su diagrama de fases. Dicha caracterización se encuentra en el capítulo (4) [55] y es la base del presente trabajo. A partir del diagrama de fases del modelo BEG, se estudiarán los efectos causados por el confinamiento, en particular las transiciones de mojado y la adsorción interfacial, haciendo uso del escaleo propuesto por Albano y Binder. 
Ambas desarrolladas en el capítulo (5). Por último, en el capítulo (6) [56], se estudiarán los efectos causados por el confinamiento sobre un modelo con desorden congelado (RBIM), es decir, un modelo en el cual las impurezas causadas por la aleatoriedad de la constante de acoplamiento se encuentren fijas, y se compararán con aquellos del modelo BEG. 


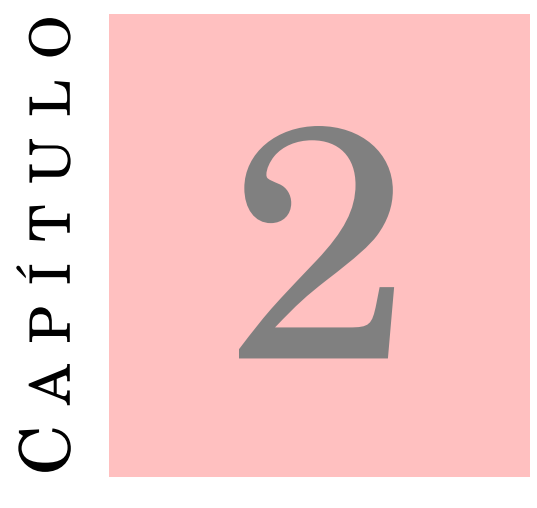

\section{Métodos Monte Carlo}

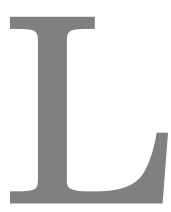

os métodos Monte Carlo (MC) son métodos numéricos, basados en los juegos de azar y en la teoría de probabilidad. Consisten en muestrear una región del espacio de fases, para estimar distintas propiedades del modelo estudiado. $\mathrm{Su}$ nombre hace referencia al casino Monte Carlo en Mónaco y su fin es dar una solución aproximada a problemas estadísticos complejos.

El uso de muestreos aleatorios para resolver problemas se remonta al año 1777 , cuando el matemático francés Georges Louis Leclerc, llevó a cabo el experimento conocido como "el experimento de la aguja de Buffon". Pero no fue hasta la década de 1940 que John von Neumann y Stanislaw Ulam [57], en medio de sus investigaciones para el Proyecto Manhattan (el cual es popularmente conocido por ser el proyecto que desarrollaría las primeras armas nucleares durante la segunda guerra mundial), dieron origen al actualmente conocido Método Monte Carlo. Durante las siguientes décadas, y gracias al 
avance de la tecnología, el método MC se convirtió en una herramienta fundamental en diversos campos de la ciencia como ser la física y la química.

Éste capítulo se desarrollará de la siguiente manera: en primer lugar se hará una breve revisión de algunos conceptos de la termodinámica y la mecánica estadística los cuales serán necesarios para la comprensión de los métodos de simulación utilizados en este trabajo. Luego se describirán ambos algoritmos: Metrópolis [46] y Wang-Landau(WL) [47, 48], y la modificación del último propuesta por Belardinelli-Pereyra[49]. Se concluirá con una discusión acerca de las ventajas y desventajas de cada algoritmo, como así también las dificultades encontradas.

\subsection{Monte Carlo y la termodinámica}

Los métodos Monte Carlo se basan en la resolución numérica de problemas que puedan ser interpretados de forma probabilística, es decir, que puedan ser transformados en problemas estocásticos. En particular, para problemas de mecánica estadística en equilibrio, los métodos Monte Carlo aproximan las soluciones exactas con el promedio obtenido a partir de un subconjunto del espacio de fases que son usados como muestreo estadístico. Entonces, ¿cómo se promedia el ensamble para obtener dicho muestreo?

Lo primero a tener en cuenta es uno de los conceptos fundamentales de la mecánica estadística: la hipótesis de ergodicidad. Esta describe un sistema dinámico que tiene el mismo comportamiento promediado en el tiempo como promediado en el espacio de fases. En otras palabras, si un determinado sistema es ergódico, sus microestados son equiprobables a lo largo del tiempo. Esto significa que el sistema alcanzará todos sus estados si se espera lo suficiente. Por tal motivo, es posible sumar la probabilidad de cada estado, en lugar de hacer la dinámica completa a lo largo de la línea de tiempo. A partir de esta hipótesis y como respuesta a la pregunta anterior, entra en juego la idea esencial de los métodos MC, el muestreo de importancia (importance sampling, en inglés). Éste se 
basa en un conjunto de procesos de Markov, conocidos como cadena de Markov [58], en los que cada estado del sistema se construye a partir de un estado anterior. Ya que cada estado tiene asociada cierta probabilidad de emerger, si se muestrean en función de sus probabilidades, estos mostrarán lo que se conoce como distribución de probabilidad. A diferencia del muestreo simple, el cual considera que todos los estados aparecen con la misma probabilidad, y por la condición de balance detallado, el muestreo de importancia elige sus estados según la distribución de Boltzmann

$$
P_{\mu}=\frac{1}{Z} e^{-\beta \mathscr{H}_{\mu}}
$$

donde $\beta=\frac{1}{k_{B} T}, k_{B}$ es la constante de Boltzmann, $\mathscr{H}_{\mu}$ es el Hamiltoniano cuando el sistema se encuentra en el estado $\mu$ y $Z$ es la función de partición de la distribución.

La mecánica estadística de equilibrio se basa en la idea de una función de partición que contiene toda la información esencial sobre el sistema en cuestión. La forma general de la función de partición para un sistema clásico es:

$$
\mathcal{Z}=\sum_{\mu_{i}} e^{-\beta \not \mathcal{H}} .
$$

Por lo tanto, la suma es sobre todos los estados posibles del sistema $\mu_{i}$, depende del tamaño del mismo y del grado de libertad de las partículas que lo componen. Una vez obtenida la función de partición, es posible realizar una conexión directa entre ésta y las cantidades termodinámicas.

El método de muestreo de importancia más utilizado, es el algoritmo de Metrópolis.

\subsection{Algoritmo de Metrópolis}

El algoritmo de Metrópolis es uno de los métodos Monte Carlo más usados en la física estadística. Los pasos ejecutados en dicho algoritmo que se detallan a continuación, se resumen mejor en el diagrama de flujo presentado en la figura (2.1). A partir de éste es posible lograr una mejor percepción conceptual de lo que el algoritmo intenta de lograr. 


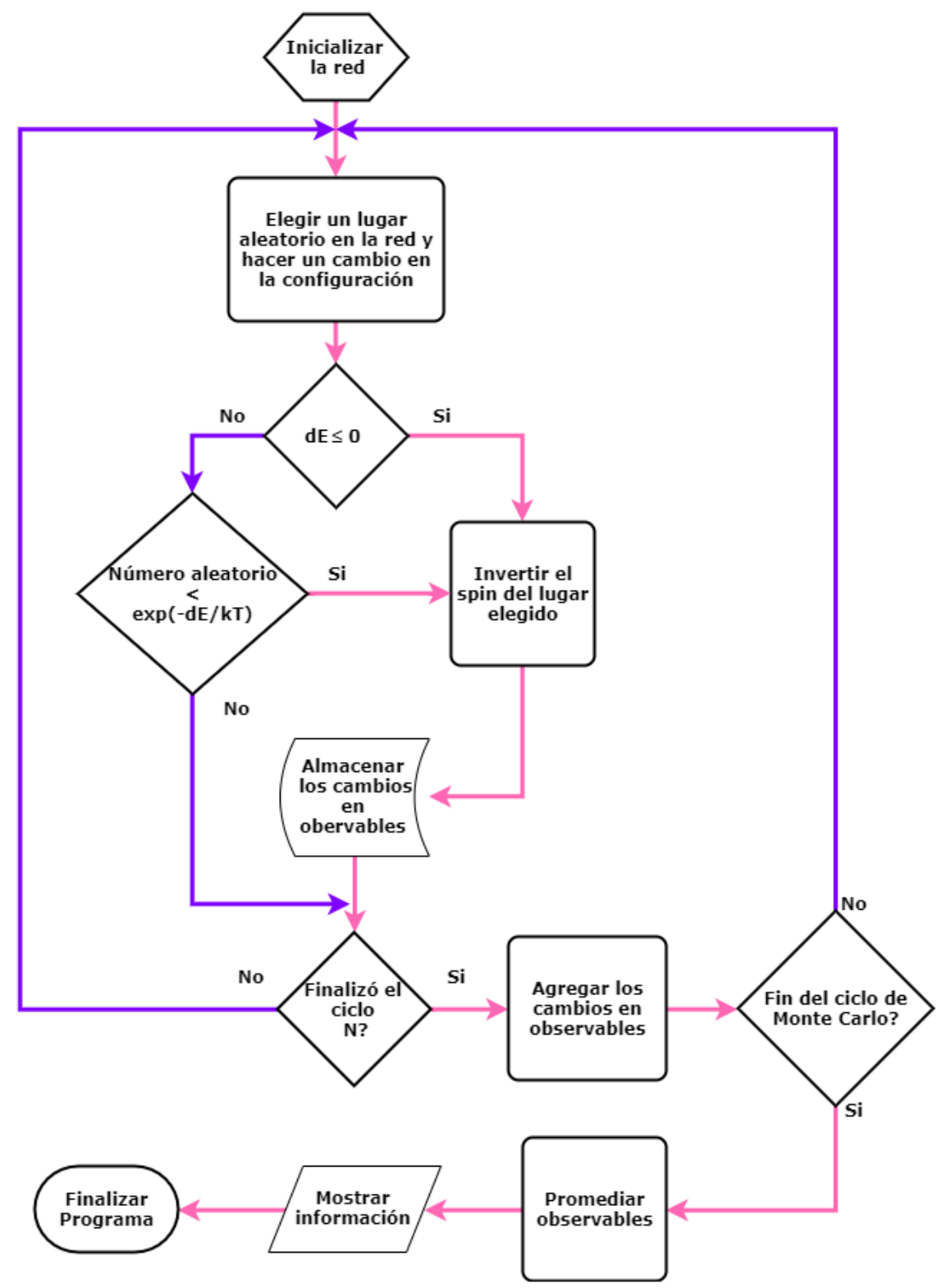

FIgURA 2.1. Diagrama de flujo del algoritmo de Metrópolis.

1. Se fija la temperatura, se elige una configuración inicial aleatoria del sistema y se calcula su energía $E_{i}$,

2. Se elige un sitio de la red de forma aleatoria y se produce un cambio en la configuración. Usualmente, en modelos con dos estados de espín, esto se realiza dando vuelta el espín que ocupa el sitio seleccionado (single spin flip, en inglés), pero en 
modelos con tres estados de espín, se sortea de forma aleatoria a qué estado pasa.

3. Se calcula la energía de la nueva configuración y se toma una decisión, dependiendo el valor de la variación de la energía:

- Si la variación de la energía es menor o igual a cero, se acepta el cambio y se guardan las modificaciones de los observables que se quieren calcular.

- Si la variación de la energía es mayor a cero, se genera un número aleatorio entre 0 y 1 y se compara con el factor de probabilidad de Boltzmann $\omega=$ $e^{-\beta \Delta E}$. Si el número aleatorio es menor, se acepta el cambio y se guardan las modificaciones de los observables. Caso contrario, se rechaza el cambio y el sistema permanece en la configuración anterior.

Los pasos 2 y 3 se repiten la cantidad de Pasos Monte Carlo (MCS, por sus siglas en inglés) que sean necesarios, donde en un MCS se busca dar vuelta un número promedio de espines, equivalente al número de sitios que conforman la red. Durante los primeros MCS, el sistema evoluciona hasta el equilibrio y si se miden los observables, estos irán cambiando. Los pasos previos al equilibrio se denominan pasos de descarte, en los cuales no se almacena la información de los observables. Una vez alcanzado el equilibrio, se colectan los datos de los observables para luego hacer el promedio.

Las variables físicas que se promedian son usualmente la energía y la magnetización como así también sus potencias. A partir de ellas, se pueden calcular cantidades como el calor específico y la susceptibilidad magnética:

$$
\begin{aligned}
& C=\frac{\beta}{T}\left(\left\langle E^{2}\right\rangle-\langle E\rangle^{2}\right), \\
& \chi=\beta\left(\left\langle m^{2}\right\rangle-\langle m\rangle^{2}\right) .
\end{aligned}
$$

Si bien es un algoritmo muy útil para el estudio de sistemas con muchas partículas, Metrópolis funciona bien si todas las configuraciones posibles del sistema se encuentran 
dentro de un rango relativamente angosto de energías, si por el contrario la separación entre las configuraciones de menor energía y la de más alta energía es muy grande, el algoritmo tenderá a quedarse atrapado en algunos de los mínimos locales del sistema, ya que el factor de Boltzmann hará que la probabilidad de escapar sea muy baja. Por otro lado, si se busca una dependencia en temperatura, es necesario repetir el ciclo tantas veces como temperaturas se necesiten.

\subsection{Algoritmo de Wang-Landau}

El algoritmo de Wang-Landau (WL) es una extensión del método Monte Carlo, propuesto por Fugao Wang y David Landau en el año 2000, pensado inicialmente para muestrear el espacio de configuraciones de ciertos sistemas que no podían ser tratados con el algoritmo de Metrópolis. Éste, mediante una exploración aleatoria, produce un histograma plano en el espacio de energías que permite calcular la densidad de estados de un sistema dado con gran precisión y sin tener ningún conocimiento previo sobre ella. Está relacionado con las técnicas denominadas Umbrella Sampling [59] y métodos de MC multicanónicos [60]. Por otro lado, es independiente de la temperatura y fácil de implementar. Sin embargo tiene un inconveniente, en algunos casos la convergencia puede ser lenta e incluso no estar asegurada.

La diferencia fundamental entre la caminata aleatoria de Wang-Landau con respecto a la de Metrópolis, es que a medida que recorre el espacio de configuraciones, se modifica la densidad de estados multiplicándola por un factor mayor a la unidad. De ésta manera, la probabilidad de volver a visitar este estado, la cual está dada por la inversa de la densidad de estados, disminuye. Esto actúa como una especie de penalización, favoreciendo a los estados con menor probabilidad de ser visitados.

El algoritmo se puede resumir en los siguiente pasos (figura(2.2)): 


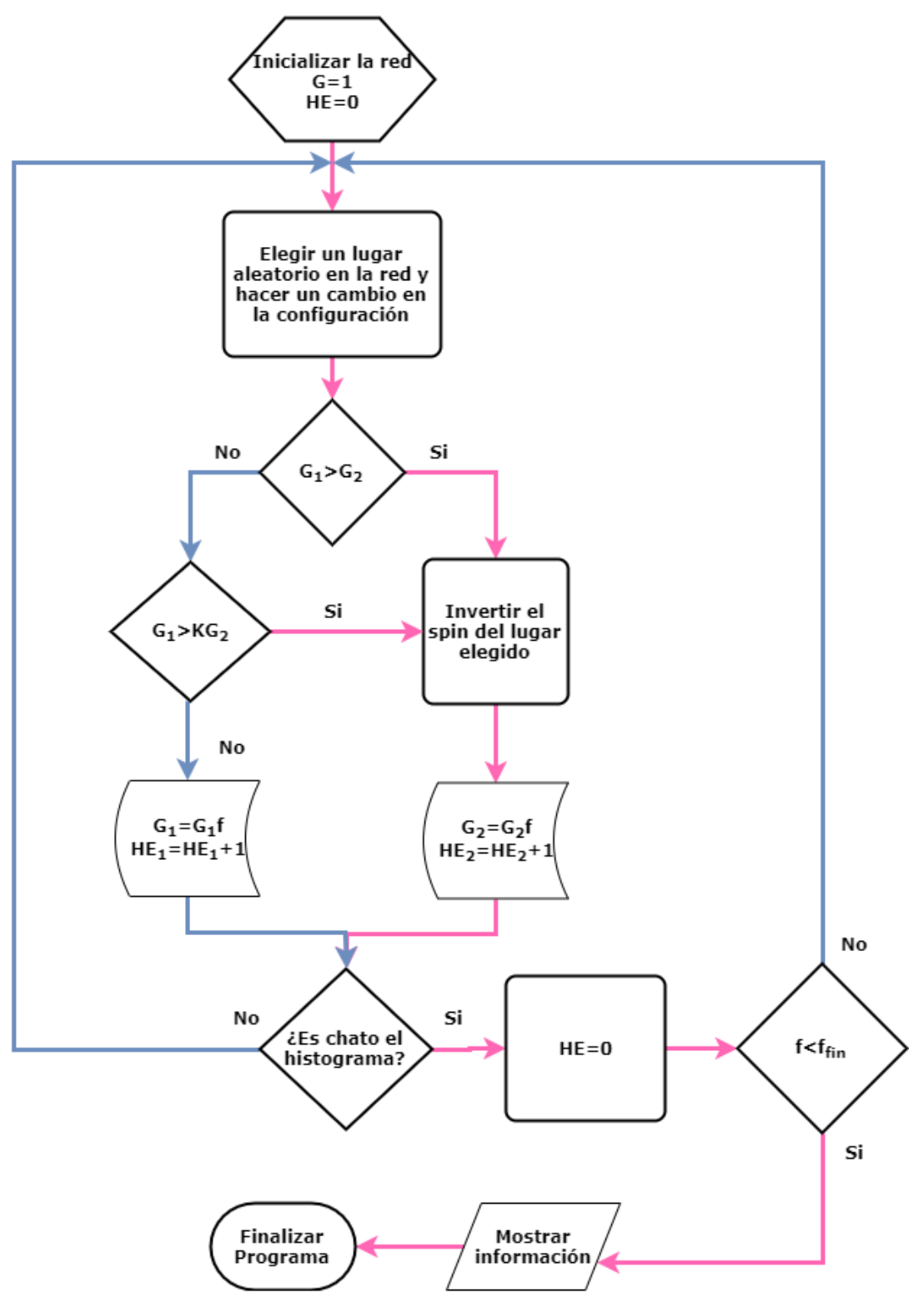

FigurA 2.2. Diagrama de flujo del algoritmo de Wang-Landau.

1. Se definen dos arreglos, los cuales corresponden a la densidad de estados del sistema, $G(E, \mathcal{N}, m)$, y al histograma en el que se irán acumulando los estados visitados, $H E(E, \mathscr{N}, m)$. Ambos dependen de la energía del sistema $(E)$, del número de sitios ocupados $(\mathscr{N})$, es decir, espines distintos de 0 , y de la magnetización $(m)$. Como el algoritmo comienza con una densidad de estados a priori desconocida, se establece $G(E, \mathscr{N}, m)=1$ y $H E(E, \mathscr{N}, m)=0$ para todos los estados del sistema. 
2. Se elige una configuración inicial aleatoria y un parámetro de refinamiento $f$. Si bien la precisión de la densidad de estados es proporcional alog $(f)$, es importante tener en cuenta que todos los estados del sistema deben ser recorridos rápidamente, por lo que un buen parámetro de refinamiento es $f=f_{0}=e$.

3. Al igual que en el paso 2 del algoritmo de Metrópolis, se elige un sitio en la red de forma aleatoria y se cambia el estado del espín que lo ocupa.

4. Considerando que $\left(E_{1}, \mathscr{N}_{1}, m_{1}\right)$ y $\left(E_{2}, \mathscr{N}_{2}, m_{2}\right)$ son los estados antes y después de dar vuelta el espín, respectivamente, se toma una decisión. A diferencia de Metrópolis, la comparación se hace sobre la densidad de estados:

- Si $G\left(E_{1}, \mathscr{N}_{1}, m_{1}\right)>G\left(E_{2}, \mathscr{N}_{2}, m_{2}\right)$ se acepta el cambio.

- Si $G\left(E_{1}, \mathscr{N}_{1}, m_{1}\right) \leq G\left(E_{2}, \mathscr{N}_{2}, m_{2}\right)$ se sortea un número aleatorio $k$, si $G\left(E_{1}, \mathscr{N}_{1}, m_{1}\right)>$ $k \times G\left(E_{2}, \mathscr{N}_{2}, m_{2}\right)$ se acepta el cambio, caso contrario se rechaza y se vuelve a la configuración anterior.

Ya sea que se acepte el cambio o no, tanto la densidad de estados como el histograma, se modifican de la siguiente manera:

$$
\begin{aligned}
G(E, \mathscr{N}, m) & =G(E, \mathscr{N}, m) \times f \\
H E(E, \mathscr{N}, m) & =H E(E, \mathscr{N}, m)+1
\end{aligned}
$$

5. Se repiten los pasos 3 y 4 hasta que el histograma se haga plano, en otras palabras, hasta que se hayan recorrido todos los estados del sistema en igual forma. Como no se puede lograr un histograma perfectamente plano, se establece algún criterio para determinar la "chatura" del mismo. En este trabajo se consideró que para todos los estados posibles del sistema, $(E, \mathcal{N}, m)$ tiene que ser mayor al $80 \%$ del promedio $\langle H E(E, \mathscr{N}, m)\rangle$ :

$$
\frac{H_{\max }-H_{\min }}{H_{\max }+H_{\min }}<0,2
$$


6. Una vez que se alcanza este criterio, se reinicia el histograma, no así la densidad de estados, y se reduce el factor de modificación según alguna función que decrezca monótonamente a 1 . Wang y Landau proponen $f_{n+1}=\sqrt{f_{n}}$, sin embargo, existen otras elecciones que aceleran la convergencia del algoritmo, las cuales se estudiarán en la siguiente sección.

La simulación termina cuando $f<f_{\text {final }}$, donde $f_{\text {final }}$ es un valor de corte prefijado, usualmente $e^{10^{-8}}$.

Dado que la densidad de estados se modifica cada vez que un estado es visitado, al final del algoritmo se obtiene una densidad de estados relativa. Para calcular los valores absolutos es necesario contar con información adicional del sistema. Por ejemplo, para el modelo de Ising con interacción ferromagnética puede utilizarse el hecho de que para el estado fundamental $G(E, \mathscr{N}, m)=2$ (todos los espines apuntando hacia arriba o todos hacia abajo), o que la suma de todos los estados accesibles es igual a $2^{N}$, donde $N$ es el número total de espines. Para un modelo con tres estados de espín, esta suma es igual a $3^{N}$.

Una vez obtenida la densidad de estados, se construye la función de partición (2.2) y a partir de ella las cantidades termodinámicas. Una de las cantidades termodinámicas fundamentales para este trabajo será la energía libre,

$$
F=-k_{B} T \log \mathcal{Z}
$$

Dado que todas las otras cantidades termodinámicas se pueden obtener a partir de la energía libre (más detalles sobre esto en el capítulo 3), esta relación provee la conexión entre la mecánica estadística y la termodinámica.

A diferencia del algoritmo de Metrópolis, es técnicamente imposible que el algoritmo de WL quede atrapado en un mínimo. Si la movida se rechaza, tanto el histograma como la densidad de estados se modifican según el estado anterior. De esta manera, 
la probabilidad de permanecer en dicho estado, disminuye a medida que se rechazan nuevas movidas. Esto genera que, tarde o temprano, la caminata progrese.

Si bien este algoritmo permite obtener toda la información que se necesita para el diagrama de fases en una sola corrida, a medida que aumentan los estados del sistema, o dicho de otra manera, a medida que se agranda la red, es más difícil que se haga plano el histograma, lo cual genera que cada simulación dure días y hasta incluso meses, dependiendo del tamaño del sistema. Esto hace que el algoritmo sea poco eficiente. Por tal motivo, Belardinelli-Pereyra proponen un cambio en el criterio de convergencia.

\subsection{WL modificado: Belardinelli-Pereyra}

Belardinelli y Pereyra propusieron una modificación al algoritmo de Wang-Landau, la cual resuelve el problema de convergencia que encuentra el mismo al trabajar con redes grandes. Para ello, proponen que el factor de modificación sea escaleado como $1 t$, donde $t$ es el tiempo Monte Carlo. Por otro lado, además de reducir el tiempo de cálculo, esta modificación reduce el error en la estimación de la densidad de estados, en comparación con el criterio de chatura del histograma.

Antes de comenzar, es necesario conocer el número de estados del sistema $\mathbb{N}$. A diferencia del algoritmo de Metrópolis, un MCS no está dado por el número de sitios de la red, sino por el número de estados posibles del sistema.

Esta modificación consiste en lo siguiente (los pasos 1, 2, 3, 4, son los redactados previamente para el algoritmo de WL, se modifica a partir del paso 5):

5. Se repiten los pasos 3 y 4 una cantidad MCS de veces. Cada MCS modifica el tiempo Monte Carlo como $t=\frac{j}{M C S}$, donde $j$ es el número de veces que el sistema intenta cambiar de estado.

6. Después de una cantidad fija de pasos Monte Carlo (por ejemplo $1000 \times M C S$ ), se 
recorre el histograma para chequear que todos los estados posibles del sistema hayan sido recorridos al menos una vez, es decir, $H(E, \mathscr{N}, m) \neq 0$. Si esto ocurre, se reduce el parámetro de refinamiento como $f_{n+1}=f_{n} / 2$ y se resetea el histograma. Caso contrario, se vuelve al paso 5 .

7. Se verifica si $f<1 / t$. En tal caso el factor de modificación pasa a ser dinámico, es decir, se modifica cada un tiempo Monte Carlo, $f=f(t)=1 / t$.

Se repiten los pasos 3 y 4 , sin almacenar nada en el histograma, mientras que $f>f_{f i n}$ donde $f_{f i n}$ es un valor prefijado al principio de la simulación. Este criterio de corte, no requiere que el histograma sea plano, sino que el factor de modificación sea menor que el valor de corte prefijado, lo cual hace mucho más rápida la simulación.

\subsection{Discusión}

Luego de trabajar con estos algoritmos, se han encontrado ciertas ventajas y desventajas de los mismos, según las condiciones de simulación a las cuales había que someter al sistema. En el capítulo 4, en el cual se estudia el modelo BEG, se utilizó el algoritmo modificado de WL con el fin de encontrar las líneas de transición de los diagramas de fases. Si bien fue posible conseguir parte de los diagramas de una sola corrida, hubo que limitar el tamaño de la red a 16×16. El motivo principal fue la formulación de la densidad de estados, la cual dependía de 3 parámetros. A medida que se agregan parámetros, la convergencia se vuelve más difícil ya que se trabaja en espacios dimensionales más grandes lo cual requiere más tiempo de cálculo. El mismo motivo llevó a usar el algoritmo de Metrópolis para el cálculo de las líneas de transición la fase cuadrupolar. Al separar la red en dos subredes, se duplicaban los parámetros de la densidad de estados y por ende, el tiempo de cálculo. En los capítulos siguientes ( 5 y 6), se utilizó el algoritmo de 
Metrópolis, ya que el de WL no presentaba ninguna ventaja sobre este, excepto en la sección 5.4, dónde se utiliza WL para obtener la energía libre del sistema. 


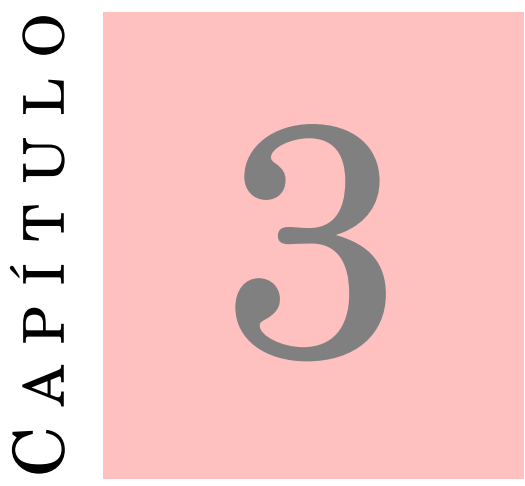

\section{MECÁNICA ESTADÍSTICA Y}

\section{TERMODINÁMICA}

L

os dos marcos teóricos que conforman hoy en día la base de cualquier estudio de fenómenos térmicos en condiciones de equilibrio (fenómenos en los cuales la energía del sistema se expresa mediante un Hamiltoniano) son la Termodinámica y la Mecánica Estadística. La primera provee una descripción macroscópica, mientras que la segunda pretende modelar estados y procesos termodinámicos a niveles microscópicos.

El objetivo principal de este capítulo es repasar algunos conceptos de la teoría de transiciones de fase y establecer notaciones básicas que serán utilizadas a lo largo de esta tesis. 


\subsection{Transiciones de Fase}

Las fases de un sistema son regiones cuyas propiedades características las diferencian de las otras regiones. Ajustando alguna variable del sistema a la cual se conoce como parámetro de control, es posible pasar de una fase a la otra. En tal caso se dice que el sistema experimenta una transición de fase.

Una forma de encontrar el punto crítico en el que ocurre dicha transición, es observando una magnitud que sea no nula en una fase y se anule al pasar a la otra. Esta magnitud se denomina parámetro de orden, cuyo nombre fue introducido por Landau en 1937 para las transiciones orden-desorden [61]. El parámetro de orden se define según el sistema físico que se esté estudiando. A modo de ejemplo se puede mencionar la magnetización espontánea para sistemas ferro-paramagnético y el volumen molar $v$ para sistemas líquido-gas. Particularmente en los siguientes capítulos, tal y como se mencionó en la sección 2.2, el parámetro de orden predilecto será la magnetización espontánea, por lo tanto, a menos que se diga lo contrario, cuando se hable de parámetro de orden, se hablará de la magnetización.

A lo largo de este trabajo se hará referencia a transiciones termodinámicas con las siguientes características:

- Transiciones de primer orden o discontinuas: Son aquellas transiciones en las que el parámetro de orden presenta una discontinuidad en el punto de transición. Estas transiciones también se caracterizan por presentar calor latente.

- Transiciones de segundo orden o continuas: En estas transiciones, el parámetro de orden cambia de manera continua, pero las derivadas de orden mayor son divergentes. Estas transiciones, como se detallará más adelante, se relacionan a su vez con la propiedad de invariancia de escala, la cual resultó ser de gran importancia a la hora de sentar las bases de una teoría para fenómenos críticos. 


\subsection{Leyes de Escala}

Como se mencionó previamente, para determinar transiciones de fase continuas se observa el parámetro de orden y el comportamiento singular de los calores específicos $(C)$, la susceptibilidad $(\chi)$, la longitud de correlación $(\xi)$, etc. Dichos comportamientos se manifiestan como leyes de potencia en las proximidades del punto crítico. Por lo tanto, en un sistema ferromagnético con campo externo $h=0$, se tiene que

$$
\begin{aligned}
& m \backsim m_{0} \tau^{\beta}, \\
& \chi \backsim \chi_{0} \tau^{-\gamma}, \\
& C \backsim C_{0} \tau^{-\alpha}, \\
& \xi \backsim \xi_{0} \tau^{-v},
\end{aligned}
$$

donde $\tau=\left|T-T_{c}\right|$ es la temperatura reducida, $\backsim$ significa asintóticamente igual y $\alpha, \beta, \gamma$ y $v$ se conocen como exponentes críticos, los cuales se detallarán más adelante. Dichas ecuaciones representan expresiones asintóticas, las cuales son válidas únicamente cuando $\tau \rightarrow 0$ (isoterma crítica), o dicho de otra manera, cuando $T \rightarrow T_{c}$.

Cuando el campo es no nulo, el parámetro de orden incrementa suavemente a medida que decrece la temperatura. Por lo tanto cuando $\tau \rightarrow 0$, y $h \rightarrow 0$, la magnetización obedece la siguiente ley de potencia

$$
m \backsim m_{0} h^{1 / \delta} .
$$

Otra cantidad importante es la función de correlación entre espines.

$$
G\left(r_{1}, r_{2}\right)=\left\langle s_{r_{1}} s_{r_{2}}\right\rangle-\left\langle s_{r_{1}}\right\rangle\left\langle s_{r_{2}}\right\rangle
$$

donde $r_{1}$ y $r_{2}$ son los vectores que apuntan a los sitios 1 y 2. Esta función describe las características espaciales de un fenómeno crítico y establece una conexión con la microscopía, ya que mide el grado de correlación entre dos partículas cualesquiera 
separadas por una distancia $r=\left|r_{1}-r_{2}\right|$. Su comportamiento varía dependiendo de su proximidad con el punto crítico. Cuando está cerca de éste, decae como

$$
G(r) \backsim r^{-(d-2)-\eta},
$$

donde $d$ denota la dimensionalidad del sistema. Mientras que cuando $\tau \gg 0$

$$
G(r) \backsim e^{-r / \xi} .
$$

Esto implica que la función decae de manera exponencial, por lo que es posible definir una distancia característica mediante el parámetro $\xi$, de modo que en estas regiones del diagrama de fases, la correlación entre espines a distancias mayores a $\xi$ resulta despreciable. Mientras que en las proximidades del punto crítico, la función se vuelve dependiente de la distancia como una ley de potencia y por lo tanto no existen longitudes características.

Es importante hacer un paréntesis para aclarar que $\xi$, se conoce como longitud de correlación (3.1) y será de gran importancia en este trabajo, particularmente en los capítulos 5 y 6 . Por tal motivo es fundamental entender su comportamiento. Para ello se considera una superficie que no es perfectamente plana, sino más bien rugosa, puede ser una pared, una interfase, etc.

Al momento de analizar la rugosidad de dicha superficie se mide el espesor de la muestra en distintas posiciones, lo cual permite obtener el ancho medio de la muestra y la desviación que presenta cada punto con respecto al mismo. Las variaciones en superficies reales usualmente tienen forma de "valles" y "montañas", por lo que la desviación con respecto a la media no cambia de forma aleatoria. De esta manera, si se chequean las desviaciones de dos medidas adyacentes, van a ser similares, mientras que si se mira la desviación en ambas puntas de la muestra, los valores no estarán relacionados. Esto quiere decir, que en algún lugar del medio hay una longitud de escala típica en la que las desviaciones con respecto a la media dejan de ser similares. Dicha longitud es la longitud 
de correlación. Por lo tanto, $\xi$ brinda información acerca de qué tan grandes son los valles y las montañas.

Retomando la discusión acerca del decaimiento de la función de correlación, cerca del punto crítico el comportamiento del sistema no se rige por detalles microscópicos del modelo sino por los exponentes críticos $\alpha, \beta, \gamma, \delta, \eta$ y $v$, los cuales reflejan propiedades esenciales del mismo, por ejemplo, la simetría de éste. Como consecuencia, todos los modelos que comparten este tipo de exponentes se comportarán de la misma forma en las cercanías del punto crítico. En estos caso se dice que dichos modelos pertenecen a la misma clase de universalidad [62]. La clase de universalidad, según Kadanoff, puede ser definida principalmente por: la dimensión espacial de la red, la simetría del parámetro de orden y el rango de interacciones $[63,64]$. También es importante considerar el número de fases que se igualan durante una transición continua.

La teoría de escala predice que éstos 6 exponentes están relacionados mediante las siguientes leyes de escala (entre otras)

$$
\begin{array}{lr}
\alpha+2 \beta+\gamma=2, & \text { (Rushbrooke) } \\
\gamma=\beta(\delta-1), & \text { (Widom) } \\
\alpha+\beta(1+\delta)=2, & \text { (Griffiths) } \\
\gamma=(2-\eta) v, & \text { (Fisher) } \\
2-\alpha=v d, & \text { (Josephson) }
\end{array}
$$

Las cuatro primeras fueron predichas desde los criterios de estabilidad termodinámicos como desigualdades. Basado en éstas evidencias, Widom [65] propuso las primeras ideas formales para explicar estas relaciones entre los exponentes críticos obtenidos de observables termodinámicos al estudiar las propiedades críticas de un fluido. La ley de Josephson, sin embargo, es especial ya que incluye la dimensión del sistema $d$. Dichas relaciones que contienen $d$, se conocen como relaciones de hiper escala y son válidas por debajo de la dimensión crítica superior $d_{c}$. 
El uso de estas relaciones es fundamental al momento de describir el comportamiento crítico de un sistema en equilibrio, ya que conociendo sólo dos exponentes críticos, se pueden determinar todos los restantes. Las predicciones de Widom sobre las propiedades críticas de un fluido, resultaron ser válidas para una gran variedad de fenómenos críticos, lo cual resultó en las primeras leyes universales de la fenomenología crítica. Para ilustrar su propuesta de una manera acorde con los fenómenos que se detallarán en esta tesis, y haciendo uso del carácter universal de estas leyes, se utilizará una descripción en el contexto de sistemas magnéticos. Por lo que la energía libre se puede escribir como:

$$
f(h, \tau)=f_{r}(h, \tau)+f_{s}(h, \tau)
$$

donde el primer término corresponde la parte regular de la energía, la cual no presenta ningún comportamiento crítico, y el segundo a la parte singular. En el punto crítico, $f_{r}(h, \tau)$ es irrelevante y $f_{s}(h, \tau)$ es una función homogénea generalizada.

Matemáticamente, una función $f\left(x_{1}, x_{2}, \ldots\right)$ es homogénea generalizada de grado $\{s\}$ si cumple la relación

$$
\lambda f\left(x_{1}, x_{2}, \ldots, x_{n}\right)=f\left(x_{1} \lambda^{s_{1}}, x_{2} \lambda^{s_{2}}, \ldots, x_{n} \lambda^{s_{n}}\right)
$$

para todo $\lambda \in(0, \infty) \mathrm{y}$, donde $s_{i}$ define el grado de homogeneidad de $x_{i}$, con $i=1, \ldots, n$. De esta hipótesis se desprenden todas las leyes de escala.

Por otro lado, teniendo en cuenta que la relación (3.8) es válida para todos los valores positivos de $\lambda$, uno puede seleccionar $\lambda$ con el fin de obtener una forma de escala. Por ejemplo, uno podría elegir $\lambda=\left|x_{1}\right|^{-1 / s_{1}}$ para obtener una expresión en la que el primer argumento del lado derecho de la ecuación (3.8) sea constante. De esta manera, la función $f$ se reduce a

$$
f\left(x_{1}, x_{2}, \ldots, x_{n}\right)=\left|x_{1}\right|^{1 / s_{1}} \tilde{f}\left(1,\left|x_{1}\right|^{-s_{2} / s_{1}} x_{2}, \ldots,\left|x_{1}\right|^{-s_{n} / s_{1}} x_{n}\right)
$$

con una función de escala $\tilde{f}$ que depende efectivamente de $n-1$ argumentos. Si se grafica $\left|x_{1}\right|^{1 / s_{1}} f\left(x_{1}, x_{2}, \ldots, x_{n}\right)$ en función de estos $n-1$ argumentos, los conjuntos de datos 
correspondientes a los distintos $x_{i}$ deberían colapsar en un mismo subespacio dado por $\tilde{f}$. A esta propiedad de los sistemas se la conoce como colapso de datos y es una característica muy importante dentro de la teoría de escala de funciones, ya que una vez identificados los parámetros de control con las variables $x_{i}$ y los exponentes críticos con $s_{i}$, el colapso ocurrirá para los valores de parámetros de control donde ocurre la transición de fase continua. Por tal motivo, a lo largo de este trabajo, se utilizará el colapso de las magnitudes físicas para estimar tanto la temperatura como los exponentes críticos.

También es importante enfatizar que, no solo los exponentes críticos son universales, sino que además lo son las funciones de escala de las variables termodinámicas correspondientes. Por ejemplo, en 1981, Bruce [66] y Binder [67] demostraron que la función de distribución de probabilidad (FDP) de los parámetros de orden es de carácter universal. Esto ha sido muy valioso en la descripción de propiedades críticas de modelos en mecánica estadística y también se ha extendido al estudio de sistemas magnéticos puros y desordenados. Debido al comportamiento universal de la FDP del parámetro de orden, esta se convierte en la huella digital de la criticalidad de cada clase de sistema. En otras palabras, si las distribuciones coinciden, no sólo los exponentes críticos pertenecen a la misma clase de universalidad, sino que también se puede determinar la temperatura crítica correspondiente.

Todas las consideraciones previas se cumplen en el límite termodinámico, es decir, cuando el volumen $V$ del sistema crece proporcionalmente al número $N$ de partículas, tal que $V \rightarrow \infty, N \rightarrow \infty$ y $\frac{N}{V}=$ cte. En este límite las fluctuaciones térmicas en cantidades globales son despreciables, y todas las cantidades termodinámicas como la energía, son simplemente funciones de variables termodinámicas como, por ejemplo, la temperatura. Pero este trabajo se basa en el uso de métodos Monte Carlo para estudiar el comportamiento de los sistemas, donde, la idea esencial detrás de un procedimiento Monte Carlo, 
es simular sistemas reales finitos generando muestras de estados en los cuales se puede encontrar el sistema. Por tal motivo, es necesario encontrar un mecanismo que permita extraer el comportamiento crítico (en el límite termodinámico) a partir de la dependencia de las cantidades físicas con el tamaño.

\subsubsection{Leyes de Escala para sistemas finitos}

Como se detalló previamente, para estudiar el comportamiento crítico de un sistema, es necesario analizar las singularidades de las variables termodinámicas que ocurren únicamente en el límite termodinámico. Sin embargo, cuando se trabaja con sistemas confinados o se efectúan simulaciones computacionales de tipo Monte Carlo, se opera con sistemas finitos. Una consecuencia directa de estas restricciones es la imposibilidad de encontrar la temperatura crítica del sistema con un simple barrido de temperaturas. De hecho, si se consideran sistemas de distintos tamaños, las curvas correspondientes al parámetro de orden y sus momentos presentarán características que pueden ser interpretadas como precursoras de la transición de fase pero ocurrirán para diferentes valores del parámetro de control. Estas observaciones sugieren que las simulaciones computacionales no son herramienta suficiente para estudiar la criticalidad de un sistema. Sin embargo, es posible superar estas dificultades haciendo uso de las leyes de escala para sistemas de tamaño finito.

Si se considera un sistema de tamaño finito con longitud característica $L$, como por ejemplo una red cuadrada de lado $L$, la parte singular de la energía libre se puede escribir en función de la misma como $f_{s}\left(\{K\}, L^{-1}\right)$, donde $\{K\}$ representa todos los campos de los cuales depende el Hamiltoniano, como la constante de acoplamiento $J$, los campos externos $h$, etc. De forma análoga a la ecuación (3.8) la energía libre escalea como

$$
f_{s}\left(\{K\}, L^{-1}\right)=\lambda^{-d} \tilde{f}_{s}\left(\{K\}, \lambda L^{-1}\right)
$$

donde $d$ representa la dimensión del sistema y $\left\{K^{\prime}\right\}$ representa las transformaciones de las 
variables $\{K\}$ mediante el grupo de renormalización. Como se puede ver, la energía libre está en función de $L^{-1}$ y no de $L$. Esto es así porque al tomar el límite termodinámico $L \rightarrow$ $\infty, L^{-1} \rightarrow 0$, y por lo tanto, queda en evidencia la no dependencia de la energía con éste parámetro. Por otro lado, es importante destacar que la validez de esta transformación sobre un sistema finito, se debe a que las transformaciones del grupo de renormalización son de carácter local, es decir, sólo involucra la vecindad de un sitio. Por tal motivo, es irrelevante el tamaño del sistema [68]. Además, si las interacciones son de corto alcance, como las que se estudiarán en este trabajo, es posible considerar que $L^{-1}$ no afecta las ecuaciones del grupo de renormalización para los parámetros $\{K\}$ [1].

Retomando el escaleo de la energía libre, se considera por simplicidad que el único parámetro de control es la temperatura reducida $\tau$. Además, cerca del punto fijo del grupo de renormalización, se puede aplicar $n$ veces la transformación, de modo tal que la aproximación lineal siga siendo válida. Por lo tanto

$$
f_{s}\left(\tau, L^{-1}\right)=\tau^{2-\alpha} F_{f}^{ \pm}\left(L^{-1} \tau^{-v}\right)
$$

donde $F_{f}^{ \pm}$es la función de escala. Teniendo en cuenta que en la proximidad del punto crítico, la longitud de correlación del sistema finito, en unidades del parámetro de red, satisface (3.1d), el argumento de $F_{f}^{ \pm}$no es más que $\left(L^{-1} \xi\right)$. Esto es importante ya que si $L \gg \xi$, el comportamiento del sistema será similar al del límite termodinámico ya que la longitud de correlación no se verá afectada por los bordes del sistema. Caso contrario, si $L \backsim \xi$ ó $L \ll \xi$, el sistema no estará gobernado por el punto crítico fijo y la longitud de correlación se verá afectada por los bordes del sistema. En consecuencia, las transiciones de fase ya no presentan singularidades de los potenciales termodinámicos en el punto crítico, sino que exhiben un pico suave de altura finita.

Finalmente, una vez conocida la forma de escalear la energía libre en función de la longitud característica del sistema, es posible escalear los otros observables físicos. Por 
ejemplo, la susceptibilidad escalearía de la siguiente forma:

$$
\begin{aligned}
\chi\left(\tau, L^{-1}\right) & =\tau^{-\gamma} F_{f}^{ \pm}\left(L^{-1} \tau^{-v}\right) \\
& =\tau^{-\gamma}\left(L^{-1} \tau^{-v}\right)^{\frac{-\gamma}{v}} \tilde{F}_{f}^{ \pm}\left(L^{-1} \tau^{-v}\right) \\
& =L^{\frac{\gamma}{v}} \tilde{F}_{f}^{ \pm}\left(\tau L^{\frac{1}{v}}\right),
\end{aligned}
$$

donde $F_{f}^{ \pm}$es la función de escaleo. El máximo de esta función, será también el máximo de la susceptibilidad. De esta manera, si el máximo se encuentra en $x_{0}$, entonces:

$$
x_{0}=\tau_{L} L^{1 / v}
$$

o bien

$$
\tau_{L}=x_{0} L^{-1 / v}
$$

donde $\tau_{L}$ se conoce como temperatura crítica efectiva del sistema finito de tamaño $L$. Es importante recordar que $\tau=\left|T-T_{c}\right|$, donde $T_{c}$ es la temperatura crítica del sistema. Lo cual implica que el desplazamiento del pico de la susceptibilidad con respecto al punto crítico depende del tamaño del sistema. De la misma manera se puede determinar la dependencia de la altura del pico con el tamaño del sistema. Para ello se evalúa la susceptibilidad en $x_{0}$

$$
\chi\left(\tau, L^{-1}\right)=L^{\gamma / v} \tilde{F}_{f}^{ \pm} \propto L^{\gamma / v}
$$

Estas dos dependencias se pueden observar de forma esquemática en la figura (3.1).

Resumiendo hasta acá, en el límite termodinámico los observables físicos presentan dependencias como leyes de potencias cerca punto crítico, con exponentes que se relacionan entre sí mediante relaciones universales. Para que eso ocurra, se requiere que la función de energía libre, tenga una parte singular que se comporte como ley de potencia al acercarse al punto crítico. De esta manera, al derivar la energía libre y comparándola directamente con la expresión para el decaimiento de los observables en cuestión, se pueden obtener las leyes de escala, que relacionan los exponentes críticos permitiendo 


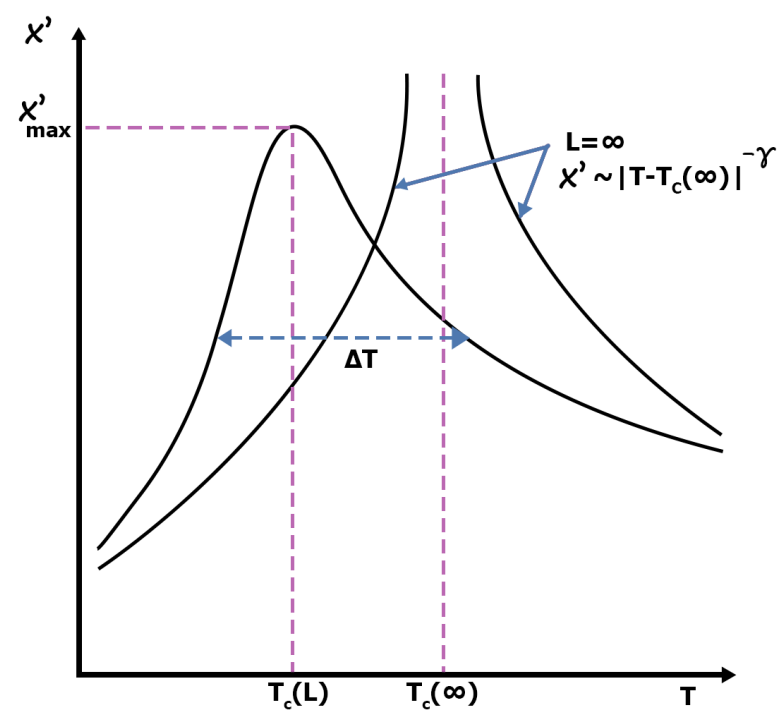

FiguRA 3.1. Comparación esquemática de la Susceptibilidad en $T_{c}$ para el límite termodinámico y para un sistema de tamaño finito.

describir el comportamiento crítico del sistema con sólo dos exponentes independientes. Los sistemas que comporten de la misma manera cerca del punto crítico o, dicho de otra manera, que compartan el mismo conjunto de exponentes críticos, pertenecen a la misma clase de universalidad. En los estudios computacionales, no es posible trabajar en el límite termodinámico por lo que se trabaja con sistemas finitos. Se realiza un escaleo de tamaño finito para caracterizar el desplazamiento del pico del parámetro de orden con respecto a la temperatura crítica. Por lo tanto, lo que resta es calcular la temperatura en el límite termodinámico.

\subsection{Determinación de la temperatura crítica en el límite termodinámico}

El análisis realizado en las secciones anteriores permite localizar los máximos de los potenciales termodinámicos y, de esa manera, asignar una temperatura crítica efecti- 
va al sistema, dada por $\tau_{L} \propto L^{-1 / v}$. Para obtener la temperatura crítica en el límite termodinámico, basta con hacer el siguiente análisis

$$
\begin{aligned}
& T_{c}(L)-T_{c}^{\infty} \propto \tau_{L} \\
& T_{c}(L)-T_{c}^{\infty}=C L^{-1 / v} \\
& T_{c}(L)=T_{c}^{\infty}+C L^{-1 / v},
\end{aligned}
$$

Esta ecuación se puede interpretar como una función lineal en términos $\operatorname{de} L^{-1 / v}$, donde $C$ es la pendiente y $T_{c}^{\infty}$ es la ordenada al origen. Esto implica que si se producen muestras del sistema de distintos tamaños, la temperatura crítica efectiva tiende de forma asintótica a la temperatura crítica en el límite termodinámico $T_{c}^{\infty}$.

\subsection{Puntos tricríticos}

En un diagrama de fases, un punto crítico marca el final de una línea de coexistencia en el que las diferentes fases coexistentes se vuelven iguales. Análogamente, un punto tricrítico se puede definir como el punto final de una línea de coexistencia de tres fases en el que las tres se vuelven idénticas. En la figura(3.2) se puede ver un diagrama de fases característico para un punto tricrítico. Este está en función del campo magnético $H$, la temperatura $T$ y del campo de ordenamiento $H^{+}$, el cual se acopla con el parámetro de orden. Cuando $H=H^{+}=0$ ocurre una transición de fase continua a la temperatura crítica $T_{N}$. En presencia de un campo magnético pequeño, la temperatura crítica disminuye pero la transición se mantiene continua, por lo que se obtiene una línea de transición $L_{2}$ que representa el límite entre las fases ordenada y desordenada. A bajas temperaturas, la presencia de un campo magnético lo suficientemente fuerte produce una transición de primer orden entre ambas fases. Por lo que se obtiene una segunda línea de transición $L_{1}$. La región ordenada del plano de simetría $H^{+}=0$ es una superficie de coexistencia $S_{0}$ que separa dos fases, en las que los parámetros de orden de las subredes son opuestos. 


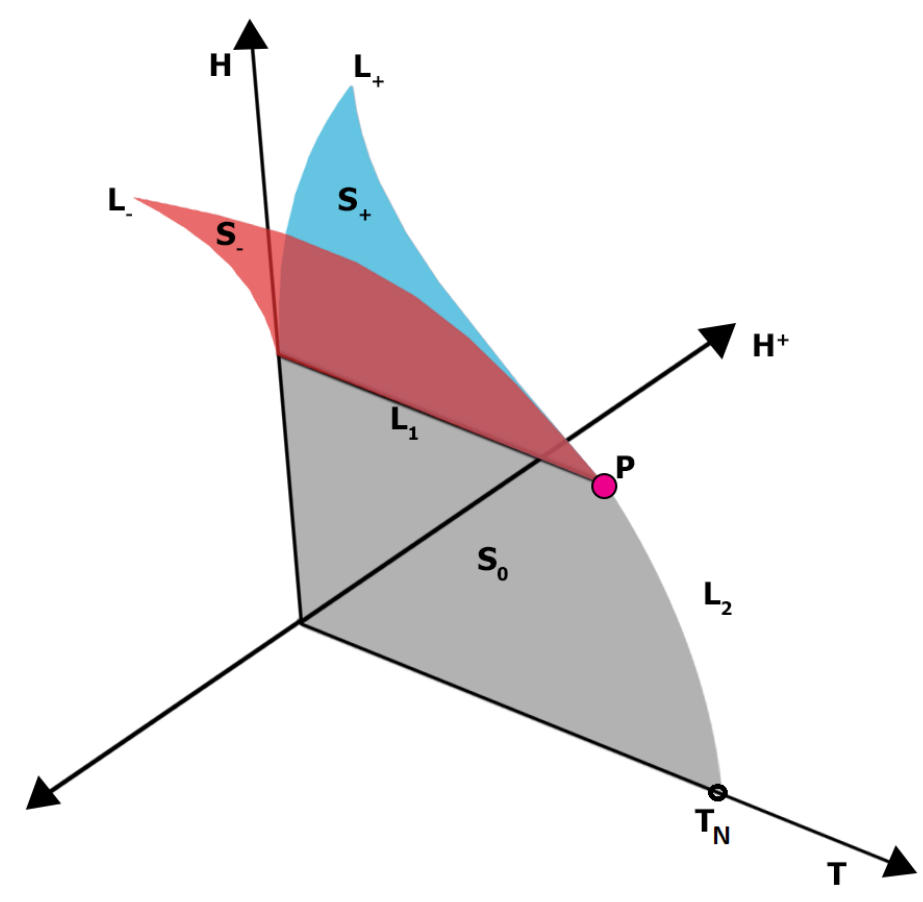

Figura 3.2. Descripción esquemática de un diagrama de fases en presencia de un punto tricrítico $P$. Este se encuentra en función de la temperatura $T$, el campo magnético uniforme $H$ y el campo de ordenamiento $H^{+}$. Un análisis detallado de las fases y los tipos de transiciones que forman parte de este diagrama, se encuentra en el texto.

La línea $L_{1}$ es, de hecho, una triple línea donde estas dos fases coexisten con la fase desordenada. A medida que aumenta la temperatura, estas tres fases se encontrarán en el punto $P$, motivo por el cual se lo conoce como punto tricrítico.

Además, la superficie $S_{0}$ está conectada a través de la triple línea a un par de superficies de coexistencia $\left(S_{+}\right.$y $\left.S_{-}\right)$los cuales se extienden simétricamente en las regiones $H^{+}>0$ y $H^{+}<0$. Cada una de estas superficies está delimitada por una línea de puntos críticos $L_{+}$y $L_{-}$, lo cuales se encuentran con $L_{1}$ en $P$.

Como se demostró en las secciones anteriores, la característica más sorprendente de los fenómenos críticos es el comportamiento singular, y a menudo divergente, de las cantidades termodinámicas a medida que se acerca el punto crítico. En muchos casos, estas son singularidades de las leyes de potencia, y la determinación de los exponentes 
críticos es una tarea central de la teoría. En el caso de una línea de puntos críticos, como los que emergen de un punto tricrítico, se espera (aunque se conocen excepciones) que toda la línea se caracterice mediante un conjunto único de exponentes universales. Los exponentes del punto tricrítico, sin embargo, son distintos de los asociados con cualquiera de las líneas críticas. Por tal motivo, es necesario llevar a cabo una nueva hipótesis de escala, similar a la llevada a cabo en la sección 3.2.

\subsubsection{Leyes de escala para los puntos tricríticos}

Los desarrollos de las teorías de escala presentados hasta ahora, se formulaban en función del modelo de Ising, el cual es un modelo particularmente "simétrico", en otras palabras, la línea de coexistencia es la línea $h=0$ y el valor del punto crítico de $h$ y $m$ es cero, figura(3.3). Dicha simetría no se encuentra presente en modelos más realistas de

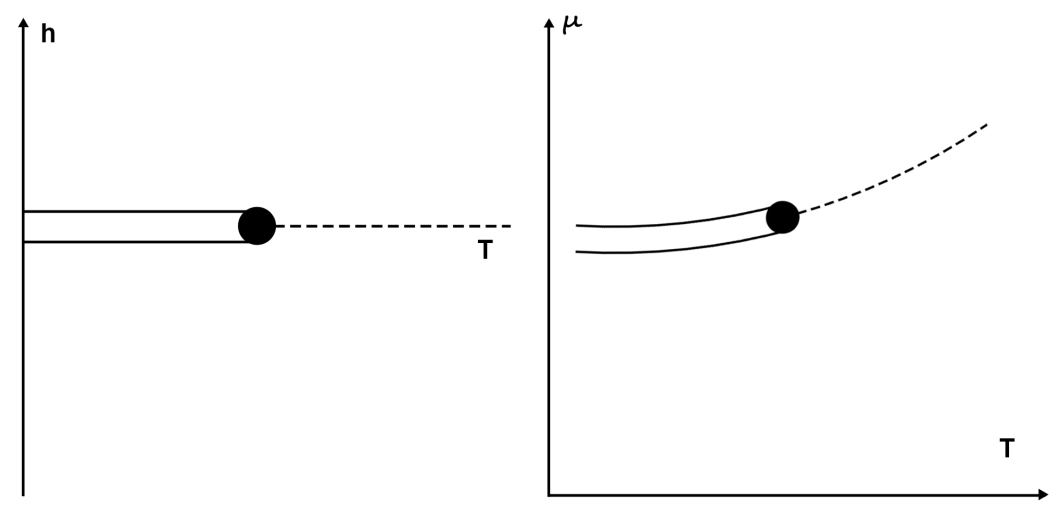

FigurA 3.3. Diferencia entre dos diagramas de fases, uno simétrico (izquierda) y uno antisimétrico(derecha). A la izquierda se observa el diagrama de fases del modelo de Ising en el plano $h-T$. Y a la derecha, el diagrama de fases de una transición Líquido-Gas en el plano $\mu-T$

fluidos y, por lo tanto, tampoco hay valores especiales de los parámetros en los puntos críticos.

Riedel y Wegner [69, 70] demostraron que la hipótesis de escala para puntos tricríticos se formula mejor en términos de campos de escala $t$ y $g$, los cuales son combinaciones 
lineales de los campos termodinámicos, determinadas, en parte, por la geometría del diagrama de fase, y desaparecen en el punto tricrítico. Por lo tanto, si se considera un diagrama como el de la figura(3.2) y se toma plano simétrico $H^{+}=0$, tal y como muestra la figura (3.4) las líneas críticas $L_{1}$ (línea punteada) y $L_{2}$ (línea llena) separan la fase ordenada de la desordenada.

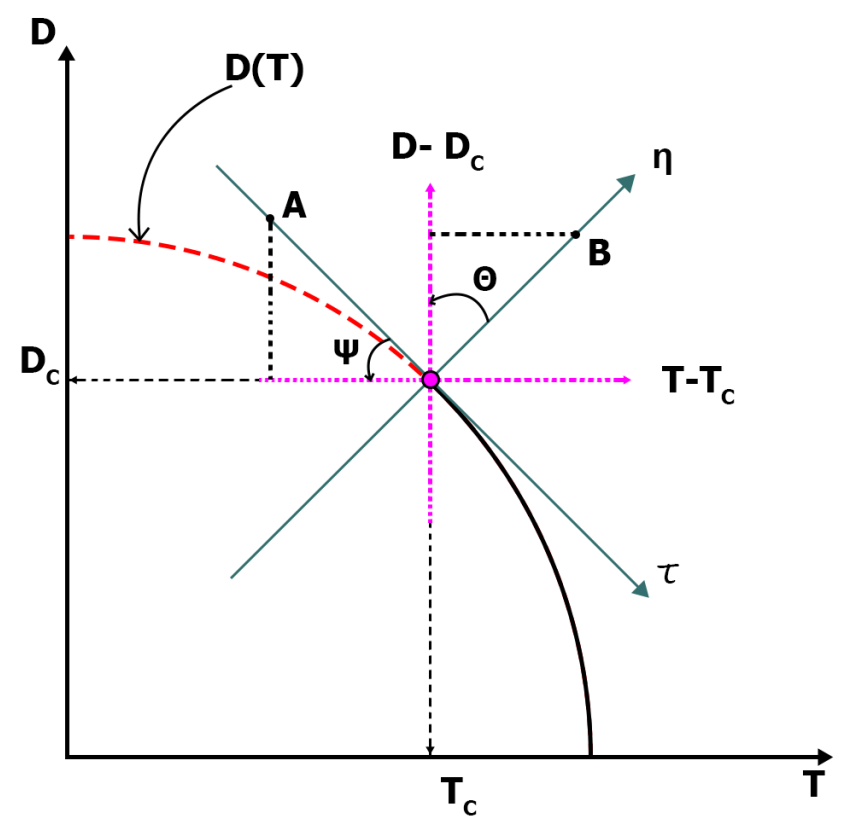

Figura 3.4. Representación de la línea de transición de primer orden $D(T)$ en el plano $H^{+}=0 .\left(T_{c}, D_{c}\right)$ es, en general, un punto tricrítico cuando se tiene una línea de transición de segundo orden. $\tau$ y $\eta$ son las direcciones de los campos de mezcla. Todos los puntos $(T, D)$ en la dirección $\tau$ (como el punto $A$ ) tienen $\eta=0$, mientras que los puntos como $B$ tienen $\tau=0$

Considerando la línea de transición de primer orden en el plano $T-D$, representada por $D(T)$, cerca del punto tricrítico se puede escribir

$$
D(T)=D_{c}-r\left(T-T_{c}\right)+\cdots
$$

donde $r=\tan (\Psi)$ (ver figura(3.3)). Despreciando los términos de orden superior

$$
\left(D(T)-D_{c}\right)+r\left(T-T_{c}\right)=0
$$


Esta ecuación representa la línea tangente a la curva $D(T)$ en el punto $\left(T_{c}, D_{c}\right)$, por lo tanto, se puede definir el primer campo de escala

$$
\eta=\left(D(T)-D_{c}\right)+r\left(T-T_{c}\right)
$$

el cual puede tener cualquier dirección no paralela a la línea tangente. De forma similar se puede definir

$$
\tau=\left(T-T_{c}\right)-s\left(D(T)-D_{c}\right)
$$

donde $s=\tan (\Theta)$. Por lo tanto $\eta=0$ define el eje $\tau$, mientras que $\tau=0$ define el eje $\eta$. Cuando $s=r=0$ se recupera la simetría $\tau=\left(T-T_{c}\right)$ y $\eta=\left(D(T)-D_{c}\right)$ (modelo de Ising).

Finalmente, se pueden utilizar estos nuevos campos para escribir la energía libre

$$
f_{s}(\tau, D)=\tau^{2-\alpha_{t}} f^{ \pm}\left(\frac{\eta}{\tau^{\beta_{t} \delta_{t}}}\right)
$$

donde $(\tau, D) \rightarrow 0 \mathrm{y} \pm$ representa el signo de $\tau$. Los exponentes $\alpha_{t}, \beta_{t}$ y $\delta_{t}$ están asociados específicamente con el punto tricrítico, por lo que se denominan exponentes tricríticos.

Análogamente a lo desarrollado en las secciones anteriores, el paso siguiente en la determinación del punto tricrítico, y su clase de universalidad, será analizar el comportamiento de un parámetro de orden $m$, y su función de distribución de probabilidad (FDP) $P(m, T)$, la cual sigue la relación [66, 67]

$$
P_{L}(m)=b L^{\beta / v} P^{*}\left(b L^{\beta / v} m\right)
$$

donde $v$ es el exponente crítico de la longitud de correlación, $b$ es una constante métrica no universal y $P^{*}$ es una función universal que permite obtener información de la clase de universalidad del punto tricrítico.

La obtención del parámetro de orden y su correspondiente FDP, como así también la obtención de los campos de mezcla $\tau$ y $\eta$ se encuentran desarrolladas detalladamente en el Apéndice B. 


\subsection{Condiciones de Contorno}

Hasta ahora, se ha analizado el comportamiento de sistemas en el límite termodinámico como así también sistemas de tamaño finito. Se han analizado casos particulares de modelos que presentan diagramas de fases simétricos como así también antisimétricos. Pero como este trabajo consiste en simulaciones, surge otra pregunta importante: ¿Cómo se tratan los bordes? Según el tipo de interacción que presenten los bordes con las partículas adyacentes, el comportamiento crítico del sistema se verá afectado o no. A continuación se presentarán las condiciones de borde que se usarán a lo largo de este trabajo.

\subsubsection{Condiciones Periódicas de Contorno}

En muchos casos, como por ejemplo en el análisis de Bulk (que se llevará a cabo en el capítulo 4 para el modelo BEG), se busca eliminar los efectos de borde. Esto se puede lograr envolviendo la red $d$-dimensional en un toro $(d+1)$-dimensional como se muestra en la figura(3.5). Esta condición de borde se conoce como Condición periódica

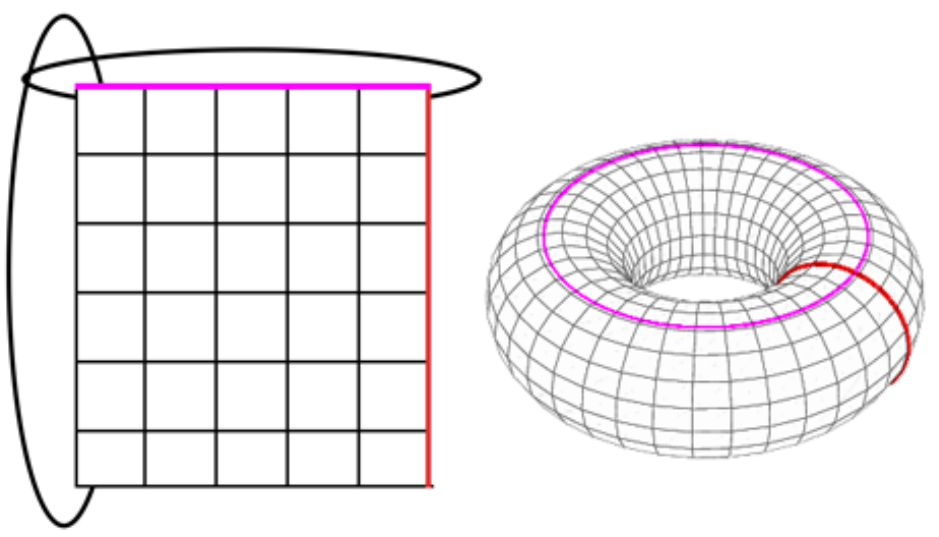

FigURA 3.5. Esquema de cómo los efectos de borde son eliminados mediante las condiciones periódicas de contorno.

de contorno tal que, el primer espín de la fila "ve" al último espín de la fila como vecino 
cercano y viceversa. Lo mismo se cumple para los primeros y últimos espines de las columnas. Este procedimiento elimina los efectos de borde, pero el sistema sigue siendo caracterizado como un sistema de tamaño finito.

\subsubsection{Condiciones Antiperiódicas de Contorno}

Esta condición funciona igual que la que la condición periódica de contorno, con la única diferencia que se revierte el signo de acoplamiento en el borde. Esto quiere decir que si el último espín de la fila tiene valor 1, el primer espín lo verá como -1. Este procedimiento se conoce como Condiciones antiperiódicas de contorno y, si bien no es útil al momento de que el sistema parezca infinito, introduce una interfase en el mismo. Usualmente se elige esta condición en una sola dirección de la red, aquella normal a la interfase que se planea estudiar, y se mantienen las condiciones periódicas de contorno en la otra dirección.

\subsubsection{Condiciones de Contorno Libres}

En esta condición el primer(último) espín de la fila no tiene ningún tipo de interacción con el último(primer) espín de la fila ni con cualquier otro espín. En otras palabras, el espín no ve ningún vecino en esa dirección. Esto se conoce como condición de contorno libre y no solo introduce efectos de tamaño finito, sino también efectos de borde. Esto provoca que, además de cambiar las dimensiones del sistema, también cambien las longitudes de correlación. En este trabajo, particularmente en los capítulos 5 y 6 , se hará uso de estas condiciones para estudiar fenómenos que involucren transiciones de localización-delocalización de una interfase, como por ejemplo las transiciones de mojado. 


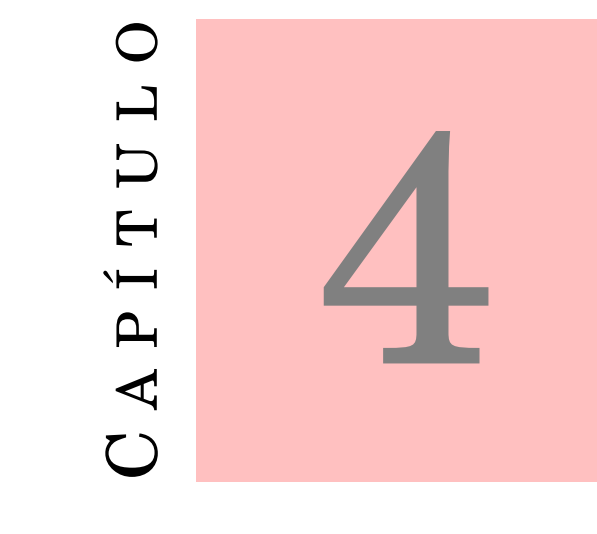

MODELO BEG

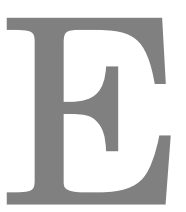

1 primer modelo a utilizar para estudiar los efectos del confinamiento es el modelo de Blume-Emery-Griffiths [30]. Este fue propuesto en 1971 por Blume, Emery y Griffiths con el fin de modelar el comportamiento termodinámico de la Mezcla de $H e^{3}-H e^{4}$ a lo largo de la línea de transición $\lambda$. Dicha línea representa el conjunto de puntos en los cuales el Helio fluido normal $H E I$ pasa a ser un super fluido $H E I I$. Este modelo es uno de los pilares fundamentales para llevar a cabo los estudios de confinamiento, por tal motivo, el primer paso es entenderlo. Entenderlo en el sentido de lograr una caracterización cualitativa y cuantitativa del mismo mediante la construcción de su diagrama de fases.

En este capítulo se presentará la caracterización del modelo BEG mediante las simulaciones Monte Carlo detalladas en el capítulo 2. La obtención del Hamiltoniano se encuentra detallada paso a paso en el Apéndice B, a los efectos de agilizar la lectura de 
este capítulo.

\subsection{El Modelo}

El modelo de BEG, consiste en una red discreta y una variable de espín $s_{i}$, la cual puede tomar valores $1,-1$ y 0 , asociada a cada sitio de la red. Solo puede haber un espín por sitio y no se permiten los sitios vacíos. El Hamiltoniano de dicho modelo tiene la siguiente forma

$$
\mathscr{H}_{B E G}=-J \sum_{\langle i, j\rangle} s_{i} s_{j}+D \sum_{i=1}^{N} s_{i}^{2}-\alpha J \sum_{\langle i, j\rangle} s_{i}^{2} s_{j}^{2} .
$$

Está basado en el modelo de Blume-Capel [28, 29] y consiste en un primer término tipo Ising, el cual regula la interacción entre los espines, donde $J$ denota la constante de acoplamiento e $\langle i, j\rangle$ indica la suma sobre los vecinos más cercanos. Un segundo término que regula la cantidad de vacancias $\left(s_{i}=0\right)$, donde $D$ es el campo cristalino y $N$ es el número total de sitios de la red. Cuando $D \rightarrow-\infty$, las vacancias desaparecen, recuperando así el modelo de Ising. El tercer término, por otro lado, corresponde exclusivamente al modelo BEG y tiene su origen en la interacción entre vacancias vecinas $\left(s_{i}=0\right)$. $\alpha$ es el parámetro que caracteriza la interacción bicuadrática, la cual es atractiva si $\alpha>0$ y repulsiva si $\alpha<0$. Los resultados que se mostrarán en este trabajo fueron obtenidos para una red cuadrada de lado $L$ con condiciones periódicas de contorno. En este caso se consideró un acoplamiento ferromagnético $(J>0)$. Sin embargo, puede ser fácilmente mapeado al caso antiferromagnético mediante la transformación que revierte el signo de la variable de espín de una de las subredes de la red cuadrada.

\subsection{Diagrama de fases para $T=0$}

El paso principal de este recorrido será calcular el diagrama de fases $D$ vs $\alpha$ para $T=0$ partiendo de argumentos energéticos simples [71]. Como se mencionó previamente, 
cuando $D \rightarrow-\infty$ las vacancias desaparecen y se recupera el modelo de Ising. Por otro lado, cuando $D \rightarrow \infty$ el numero de vacancias aumenta hasta que se llena el sistema de éstas. Si bien es un estado de espín 0, se llamará a esa fase "paramagnética" ya que si se le aplica un campo magnético se magnetiza linealmente. Por lo tanto:

- Para $D \rightarrow \infty$ (fase paramagnética)

$$
E \rightarrow 0
$$

- Para $D \rightarrow-\infty$ (fase ferromagnética)

$$
E \rightarrow-2 J+D-2 \alpha J
$$

En el momento de la transición de fase paramagnética $\leftrightarrow$ ferromagnética

$$
\begin{aligned}
& 0=-2 J+D-2 \alpha J \\
& D=2 J(\alpha+1)
\end{aligned}
$$

Por lo tanto, para $\alpha \geq-1$ hay un solo estado ordenado: una fase ferromagnética. Pero un número suficiente de vacancias puede llevar al sistema a un estado paramagnético desordenado a lo largo de la línea dada por (4.4). Cabe destacar, que para el caso particular de $\alpha=0$ se recupera el ya mencionado modelo de Blume-Capel, el cual tiene un solo punto crítico para temperatura cero en $D=2 J$.

Para $\alpha \leq-1$ hay una fuerte repulsión entre las vacancias vecinas, de modo que a medida que el campo $D$, fuertemente negativo, disminuye en intensidad y las vacancias comienzan a aparecer, tienden a llenar una de las subredes disjuntas de la red cuadrada; esta es la fase cuadrupolar cuyo análisis energético es

$$
E \rightarrow \frac{D}{2}
$$


Por lo tanto la transición de fase ferromagnética $\leftrightarrow$ cuadrupolar se va a dar a lo largo de la línea

$$
\begin{aligned}
& \frac{D}{2}=-2 J+D-2 \alpha J \\
& D=4 J(\alpha+1) .
\end{aligned}
$$

Por último, la transición de fase paramagnética $\leftrightarrow$ cuadrupolar

$$
0=\frac{D}{2} \Rightarrow D=0
$$

Es evidente que esta fase también se desestabiliza por la proliferación de vacancias, de modo que incluso en $T=0$, el sistema eventualmente se desordena hacia un estado paramagnético por encima de $D=0$. Estos resultados se pueden ver de forma esquemática en la figura (4.1)

$$
\underline{\mathbf{T}=\mathbf{0}}
$$

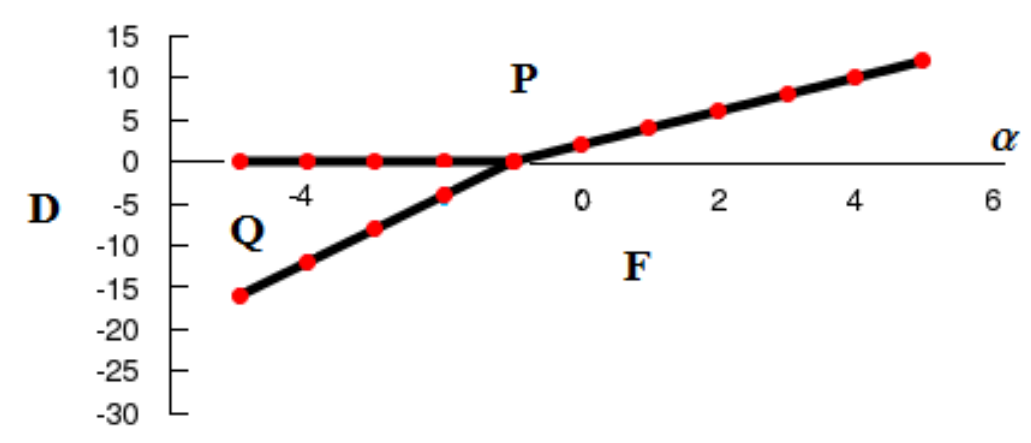

Figura 4.1. Diagrama de fases del modelo BEG para $T=0$, en función de campo cristalino $D$ y la interacción bicuadrática $\alpha$. Consta de tres fases: ferromagnética $(F)$, paramagnética $(P)$ y cuadrupolar $(Q)$.

\subsection{Diagrama de fases para $T \neq 0$}

En esta sección, se va a analizar el comportamiento del sistema para temperaturas distintas de cero con el fin de construir un diagrama de fases no sólo en función de $D$ 
y $\alpha$, sino también, en función de la temperatura $T$. Aunque parezca paradójico, luego de analizarlo detenidamente se concluyó que para un mejor entendimiento del proceso, conviene empezar el análisis mostrando el diagrama de fases en primer lugar. El mismo se muestra en la figura(4.2) y condensa los resultados principales de éste capítulo.

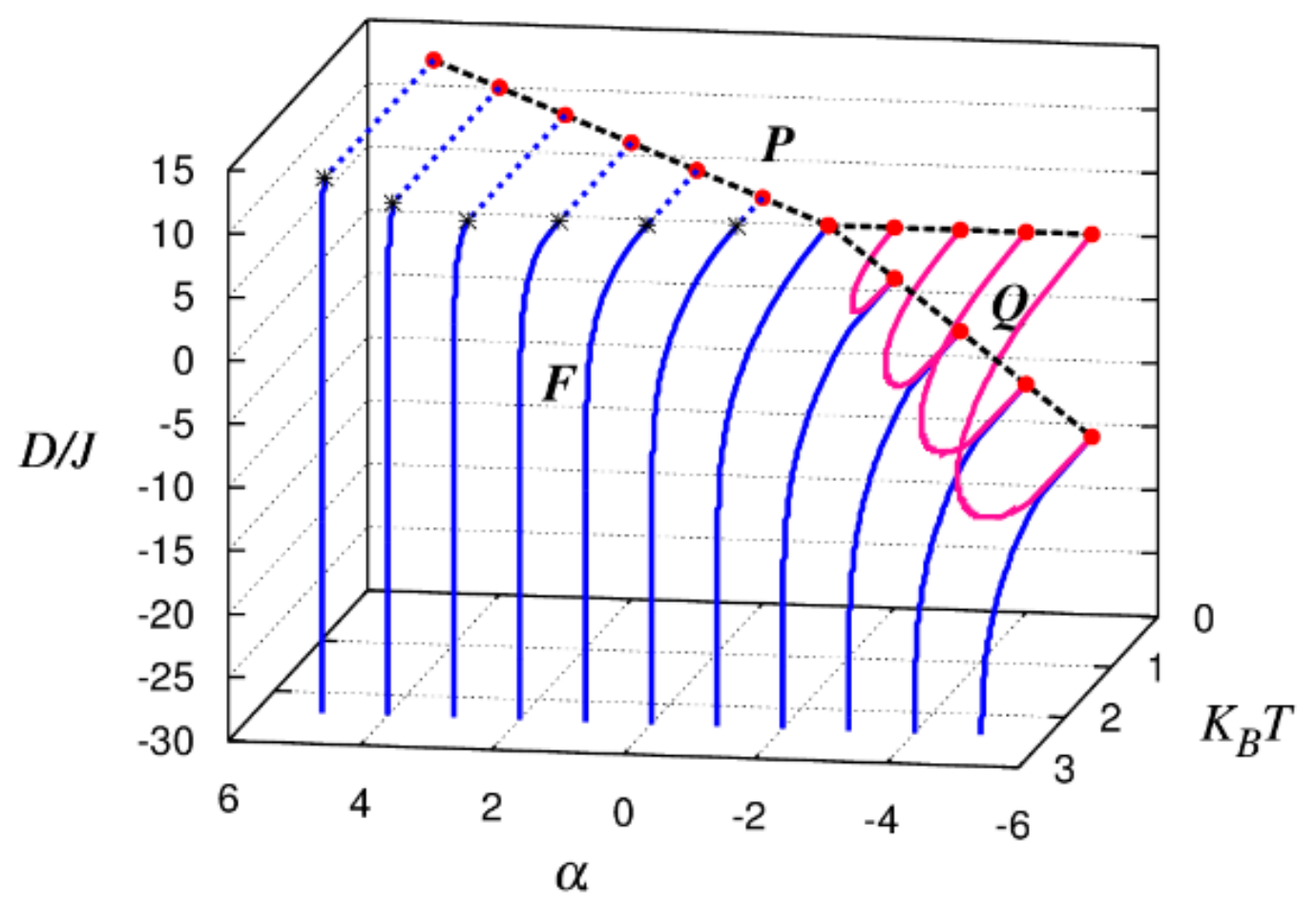

FigurA 4.2. Diagrama de fases del modelo BEG. Muestra las tres fases: ferromagnética, paramagnética (azul) y cuadrupolar (rosa), separadas por líneas de transición. Las líneas de transición ferromagnética $\leftrightarrow$ paramagnética presentan transiciones de primer orden (línea punteada) y segundo orden(línea completa), separadas por puntos tricríticos (estrellas). También muestra la línea de transición del estado fundamental (línea punteda negra) y el estado fundamental para cada valor de $\alpha$ (puntos rojos).

El diagrama de fases del modelo BEG en función de $D, \alpha$ y $T$, muestra la existencia de tres fases diferentes: una fase paramagnética desordenada $(P), \mathrm{y}$ dos fases ordenadas: una ferromagnética $(F)$ y otra cuadrupolar $(Q)$. La transición entre la fase paramagnética y la ferromagnética muestra una línea de puntos tricríticos (marcados con estrellas 
negras) la cual separa las transiciones de primer orden (línea punteada) y las de segundo orden (línea llena). Ésta línea termina en $T=0$, en el punto donde las tres fases coexisten. La transición en la fase cuadrupolar es siempre de segundo orden.

La existencia de la fase ferromagnética se determina fácilmente utilizando la magnetización $m=\frac{1}{N} \sum_{i_{1}}^{N} s_{i}$ y la susceptibilidad $\chi$. También hay indicios de la transición en el calor específico, pero a menudo se ven opacadas por la anomalía de Schottky asociada con la anisotropía del sistema.

El panel superior de la figura(4.3) muestra cómo se comporta la magnetización en función de la temperatura al atravesar una transición de fase de segundo orden para $D=3$ y $\alpha=1$. Los diferentes símbolos representan los distintos tamaños de red. Se puede ver que a medida que la temperatura disminuye, hay un claro aumento en la magnetización, la cual se vuelve cada vez más aguda a medida que aumenta el tamaño de la muestra. En el inset se puede ver el colapso de los puntos mediante el uso de los exponentes críticos, en términos de las variables reducidas $M L^{\beta / v}$ y $\left|T-T_{c}\right| L^{1 / v}$ con $v=1$ y $\beta=1 / 8$, tal y como se desarrolló en el capítulo 3 . El colapso de los puntos se usa para encontrar la temperatura crítica $T_{c}^{\infty}=1,748(2)$ y mostrar que la transición pertenece a la clase de universalidad de Ising en $2 D$, lo cual es coherente con resultados en trabajos anteriores [39]. Esta figura se construyó usando el algoritmo de Metrópolis a fin de comparar y verificar los resultados obtenidos con el algoritmo de Wang-Landau y aquellos obtenidos mediante el estudio de tamaño finito del sistema. Ambos métodos coinciden con un error menor al $1 \%$.

Como se ha mencionado anteriormente, la línea de transición pasa de primer a segundo orden en el punto tricrítico. Para determinar la ubicación de la temperatura crítica de segundo orden en el límite termodinámico, se puede hacer uso del cumulante de cuarto orden de Binder

$$
U=1-\frac{\left\langle m^{4}\right\rangle}{3\left\langle m^{2}\right\rangle^{2}}
$$



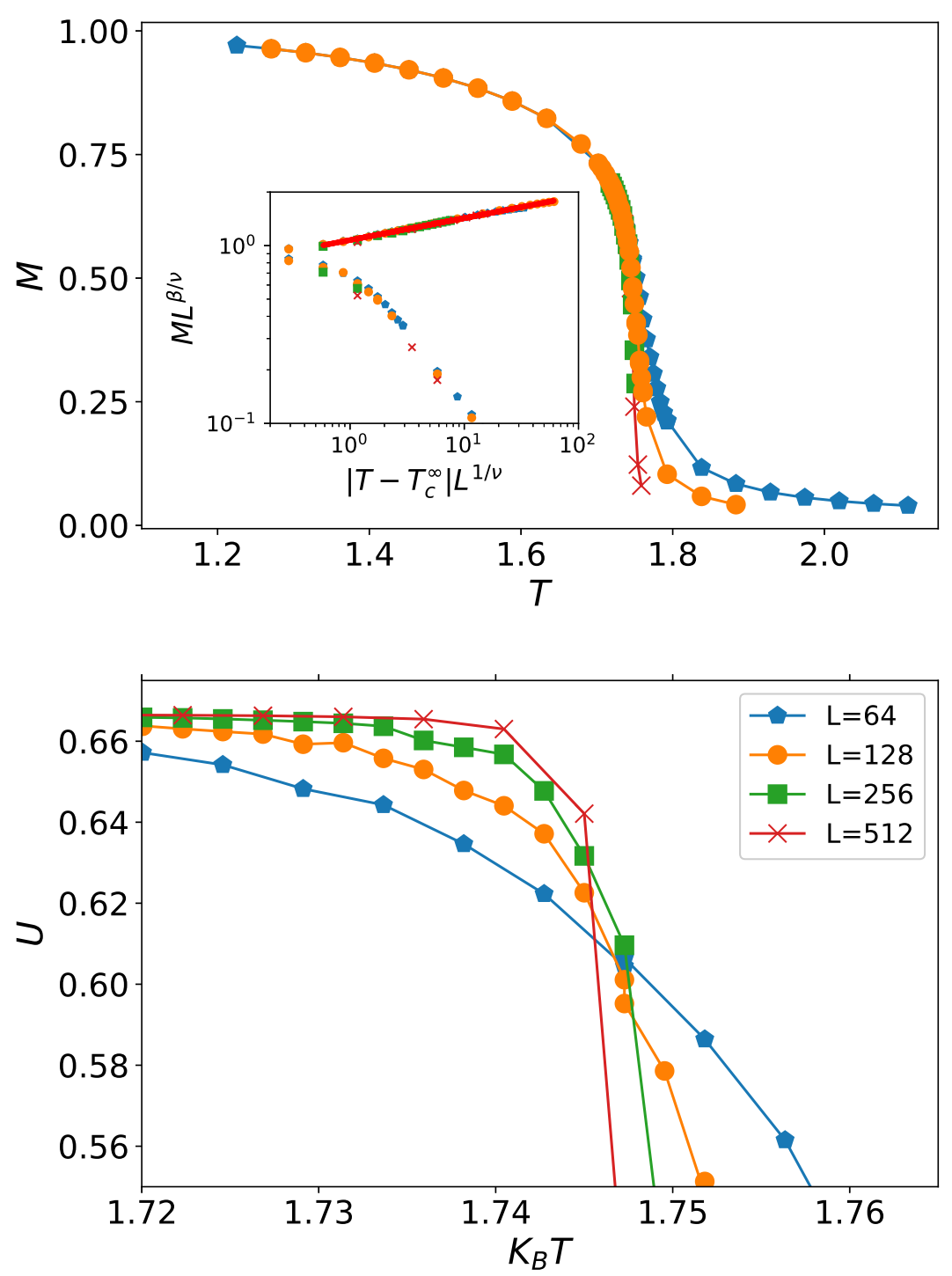

FigURA 4.3. Panel superior: gráfico de la magnetización $\langle|m|\rangle$ vs la temperatura $k_{B} T$. Se muestran los tamaños red y los parámetros utilizados son $\alpha=1 \mathrm{y}$ $D=3$. Inset: gráfico de escala de $M L^{\beta / v}$ versus $\left|T-T_{c}^{\infty}\right| L^{1 / v}$, que muestra el colapso de datos obtenido al tomar $T_{c}^{\infty}=1,748(2)$. Si se ajustan estos datos, es posible recuperar $\beta$ de la pendiente de la rama $T>T_{c}^{\infty}$ (ver línea completa). Panel inferior: gráficos del cumulante $U$ vs $k_{B} T$ obtenidos para redes de diferente tamaño y para la misma elección de $D$ y $\alpha$, los cuales muestran el punto de intersección común a la temperatura crítica dada por $T_{c}^{\infty} \simeq 1,748(2)$, de acuerdo con el valor utilizado para obtener colapso de los datos (panel superior). 
el cual escalea de manera invariante en la temperatura crítica y por lo tanto muestra un punto de intersección, en el que se cruzan todas las curvas independientemente del tamaño. La solución del escaleo para el cumulante carece de cualquier factor dependiente del tamaño, por lo tanto

$$
U(L) \backsim \tilde{U}\left(\left(T-T_{c}^{\infty}\right) L^{1 / v}\right)
$$

donde $\tilde{U}$ es una función de escala que no se especificará aquí. De esta manera, el punto de intersección de las curvas correspondientes a muestras de distintos tamaños permite estimar la temperatura crítica. El panel inferior de la figura (4.3) muestra la temperatura crítica de segundo orden, en este caso $T_{c}^{\infty}=1,748(2)$.

\subsection{Transiciones de primer orden y puntos tricríticos}

En la figura(4.4) se puede ver un claro ejemplo de cómo la transición del estado paramagnético al ferromagnético en función del campo cristalino $D$, cambia de primer orden para bajas temperaturas a segundo orden a medida que la temperatura aumenta. Esto es evidente al ver cómo la discontinuidad en $m$ se va suavizando cuando se mantiene un valor fijo de $\alpha$ para distintas series de temperaturas.

Para determinar la línea de primer orden y la posición del punto tricrítico, se hará uso del método propuesto por Wilding y Bruce [72], que posteriormente varios autores utilizaron con éxito [73-76]. Dicho método está detallado en el apéndice A y consiste en calcular la función de distribución de la probabilidad $P(\mathscr{D})$, de un nuevo parámetro de orden $\mathscr{D}$ definido como $\mathscr{D}=\mathscr{N}-s E$, donde $s$ es un parámetro que representa la asimetría de los campos de escala en el punto de transición, y depende no sólo de la ubicación exacta sobre la línea de coexistencia sino también en el tamaño del sistema.

Haciendo uso del algoritmo de WL se pueden obtener los valores $\mathscr{N}, E$ y $P(\mathscr{N}, E)$. 


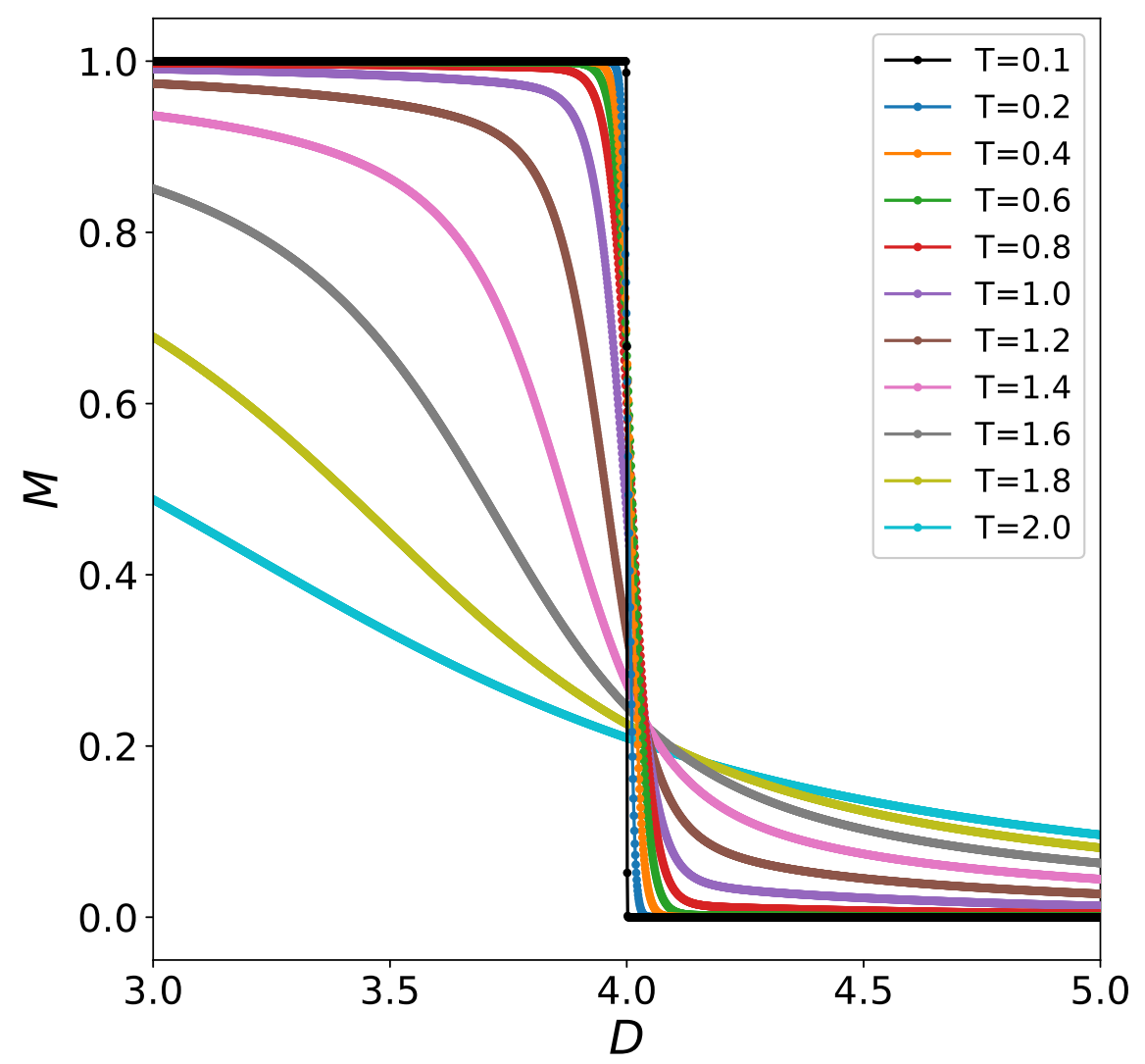

FiguRA 4.4. Gráfico de la magnetización $\langle|m|\rangle$ vs el campo cristalino $D$, para $L=16$ y $\alpha=1$.

Por otro lado, como estos estudios se llevan a cabo en la vecindad del punto tricrítico, se considera $s=0,5$, Con esos datos es posible armar el nuevo parámetro de orden $\mathscr{D}$ y calcular su FDP $P(\mathscr{D})$ ajustando $T$ y $D$.

Hay muchos criterios computacionales que se podrían adoptar para encontrar la línea de transición de primer orden (y por lo tanto el punto tricrítico, el cual representa el punto final de dicha línea). Motivados por el uso del carácter universal de la FDP y siguiendo la metodología de Wilding y Bruce [72] se utilizará el criterio de que la línea de coexistencia entre ambas fases es el conjunto de puntos en el espacio $D-T$ a lo largo del cual la FDP, $P(\mathscr{D})$, presenta dos picos de igual altura. Esta condición representa el análogo al tamaño finito de la igualdad de energía libre que caracteriza el borde de la 
fase en el límite termodinámico [77].

En la figura(4.5) se puede ver dicha función en la vecindad del punto tricrítico para $\alpha=1$.

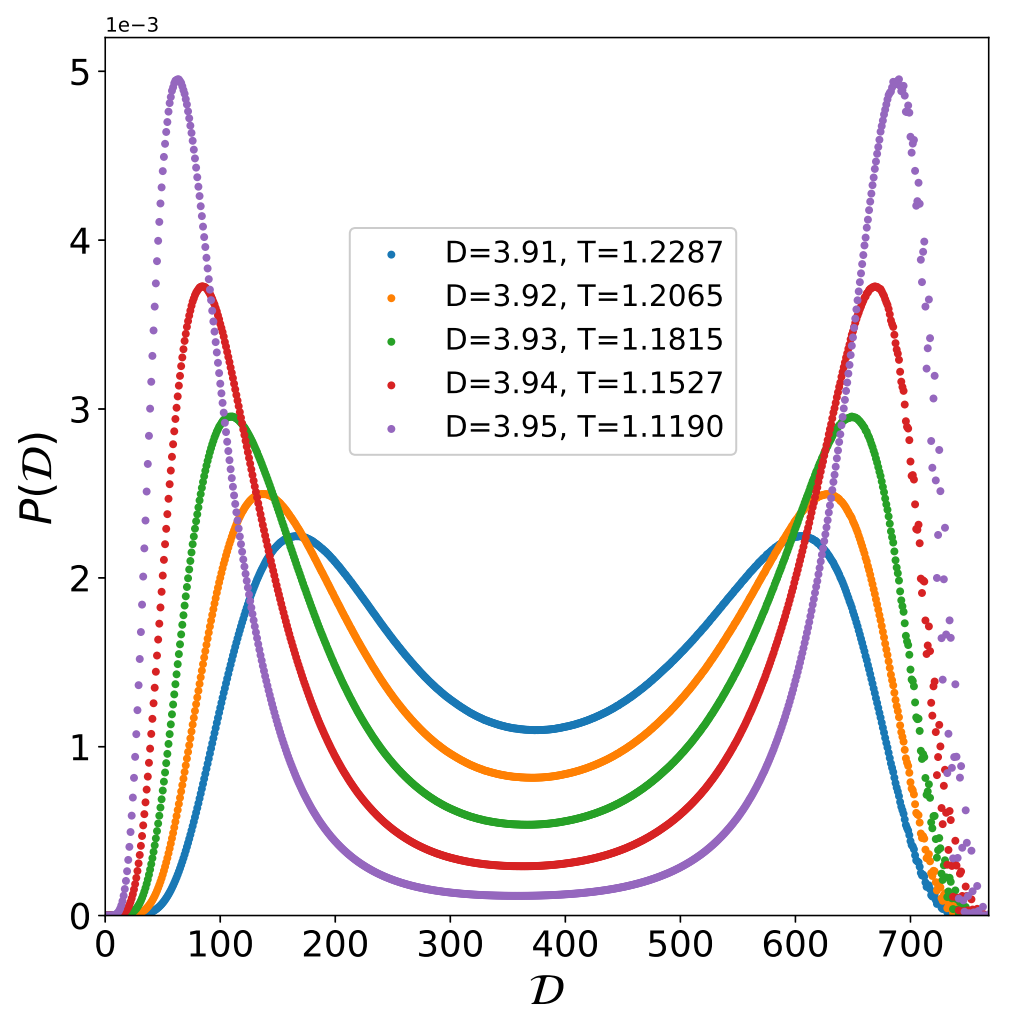

FigurA 4.5. Función de la distribución de la probabilidad de $\mathscr{D}$ para $\alpha=1 \mathrm{y}$ $L=16$, la cual presenta dos picos de la misma altura para diferentes valores de $\mathscr{D}$ y de $T$.

En la región de primer orden, lo picos se encuentran separados, pero a medida que se aproximan a la región de segundo orden, dichos picos se empiezan a unir hasta formar finalmente un sólo pico. Esto implica que el sistema está experimentando un cambio en el carácter de la transición, por lo tanto es necesario adoptar un criterio para determinar los puntos tricríticos para tamaños finitos. Este consiste en comparar la separación de los picos cuando el valor más bajo de $P(\mathscr{D})$ representa el $0,5 \%$ de la altura. De esta manera, aquellas funciones que cumplan dicho criterio serán consideradas de primer orden, mientras que aquellas que superen el 0,5\%, serán consideradas de segundo. 
Una forma adicional de verificar la ubicación del punto tricrítico es haciendo uso del método de la intersección del cumulante. Como se mencionó previamente, la invariancia de escala de la función $P(\mathscr{D})$, implica que en el punto tricrítico, los valores del cumulante para distintos tamaños del sistema deberían ser iguales, presentando un punto de intersección independiente del tamaño. Por lo tanto, los pasos a seguir son: estimar el campo cristalino tricrítico $D_{t}$ mediante la extrapolación de los puntos tricríticos para redes de distintos tamaños, como se muestra en la figura (4.6), haciendo uso de los exponentes tricríticos $1 / v=1,86(2)$ y $\beta=0,0453(2)$ [78].

Luego, se obtiene la temperatura tricrítica a partir del punto de intersección de los cumulantes de redes de distintos tamaños, medidos para $D_{t}$. La estimación del punto tricrítico para $\alpha=0$ es $D=1,966(1)$ y $T=0,608(1)$. Es importante destacar que la estimación del error es puramente gráfica ya que los cálculos están basados en un muestreo de la densidad de estados obtenida con el algoritmo de WL. Este valor coincide con valores obtenidos previamente para el punto tricrítico del modelo Blume-Capel en redes cuadradas (Tabla(4.1)):

\begin{tabular}{|l|l|l|}
\hline \hline \multicolumn{3}{|c|}{ Puntos Tricríticos para $\alpha=0$} \\
\hline$T_{t}^{\infty}$ & $D_{t}$ & Referencia \\
\hline $0,608(1)$ & $1,966(1)$ & Luque et al. [55] \\
\hline $0,610(5)$ & $1,965(5)$ & {$[79]$} \\
\hline $0,608(1)$ & $1,9665(3)$ & {$[75]$} \\
\hline $0,609(4)$ & $1,965(5)$ & {$[80]$} \\
\hline $0,609(3)$ & $1,966(2)$ & {$[81]$} \\
\hline $0,608(1)$ & $1,9665(3)$ & {$[73]$} \\
\hline
\end{tabular}

Tabla 4.1: Comparación del punto tricrítico obtenido para $\alpha=0$ con aquellos previamente obtenidos por otros autores.

Los puntos tricríticos encontrados en este trabajo para distintos valores de $\alpha$, se encuentran agrupados en la tabla(4.2). Las variaciones que presentan estos puntos causadas por el tamaño de la red son menores al $1 \%$.

Una vez obtenidos los valores tricríticos $D_{t}$ y $T_{t}$, es posible realizar un análisis de 


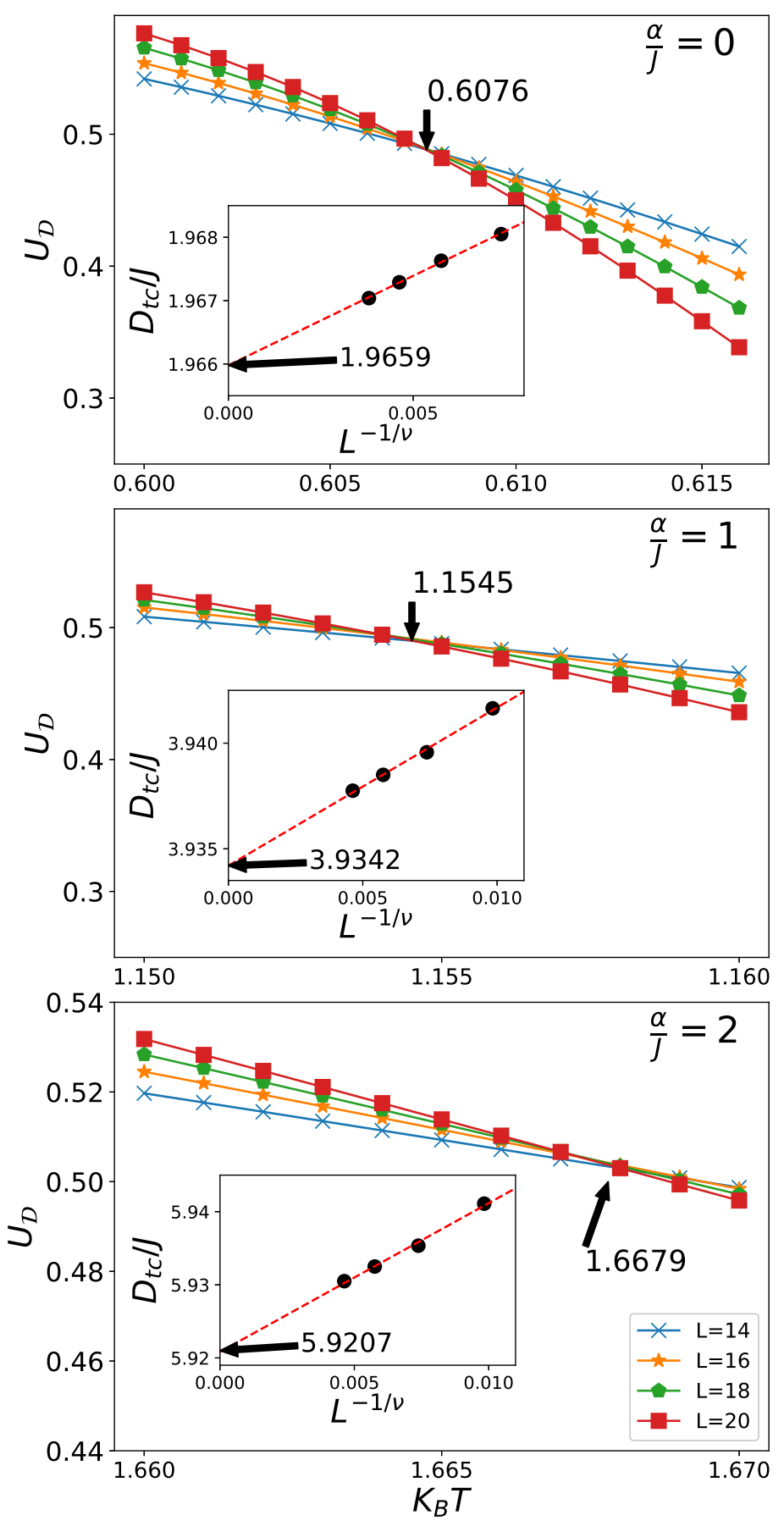

FIgURA 4.6. Ubicación del punto tricrítico para $\alpha=0, \alpha=1$ y $\alpha=2$. La temperatura tricrítica se determina en el punto de intersección del cumulante de cuarto orden, mientras que la extrapolación de los puntos de transición con $1 / v=1,86$ proporciona una estimación del campo cristalino tricrítico (recuadro). Los tamaños de las redes son 14, 16, 18, 20. 


\begin{tabular}{|l|l|l|}
\hline \hline \multicolumn{3}{|c|}{ Puntos Tricríticos } \\
\hline$\alpha$ & $T_{t}$ & $D_{t}$ \\
\hline 0 & $0,608(1)$ & $1,966(1)$ \\
\hline 1 & $1,154(1)$ & $3,934(1)$ \\
\hline 2 & $1,668(1)$ & $5,921(1)$ \\
\hline 3 & $2,095(1)$ & $7,940(1)$ \\
\hline 4 & $2,303(1)$ & $9,979(1)$ \\
\hline 5 & $2,348(1)$ & $11,980(1)$ \\
\hline
\end{tabular}

Tabla 4.2: Puntos tricríticos obtenidos para distintos valores de $\alpha$ en el modelo BEG.

tamaño finito. Para ello se considera la variable $\tilde{\mathscr{D}}=\mathscr{D}-\langle\mathscr{D}\rangle$ lo cual permitirá tener promedio cero y varianza unitaria. La FDP de esta nueva variable se puede obtener a partir de la solución estimada del escaleo de tamaño finito [75]

$$
P_{L}(\tilde{\mathscr{D}})=L^{(d-1 / v)} P_{\mathscr{D}}^{*} L^{(d-1 / v)} \tilde{\mathscr{D}}
$$

donde $P^{*}$ es una función universal y $d$ es la dimensión de la red. Como se muestra en el inset de la figura (4.7), las FDP para distintos tamaños de red y exponente tricrítico $1 N$, colapsan perfectamente. El posible error en esta estimación, se origina principalmente en la naturaleza discreta de $Q$, especialmente para sistemas pequeños, esto puede causar ambigüedad en la determinación de dicho parámetro. 


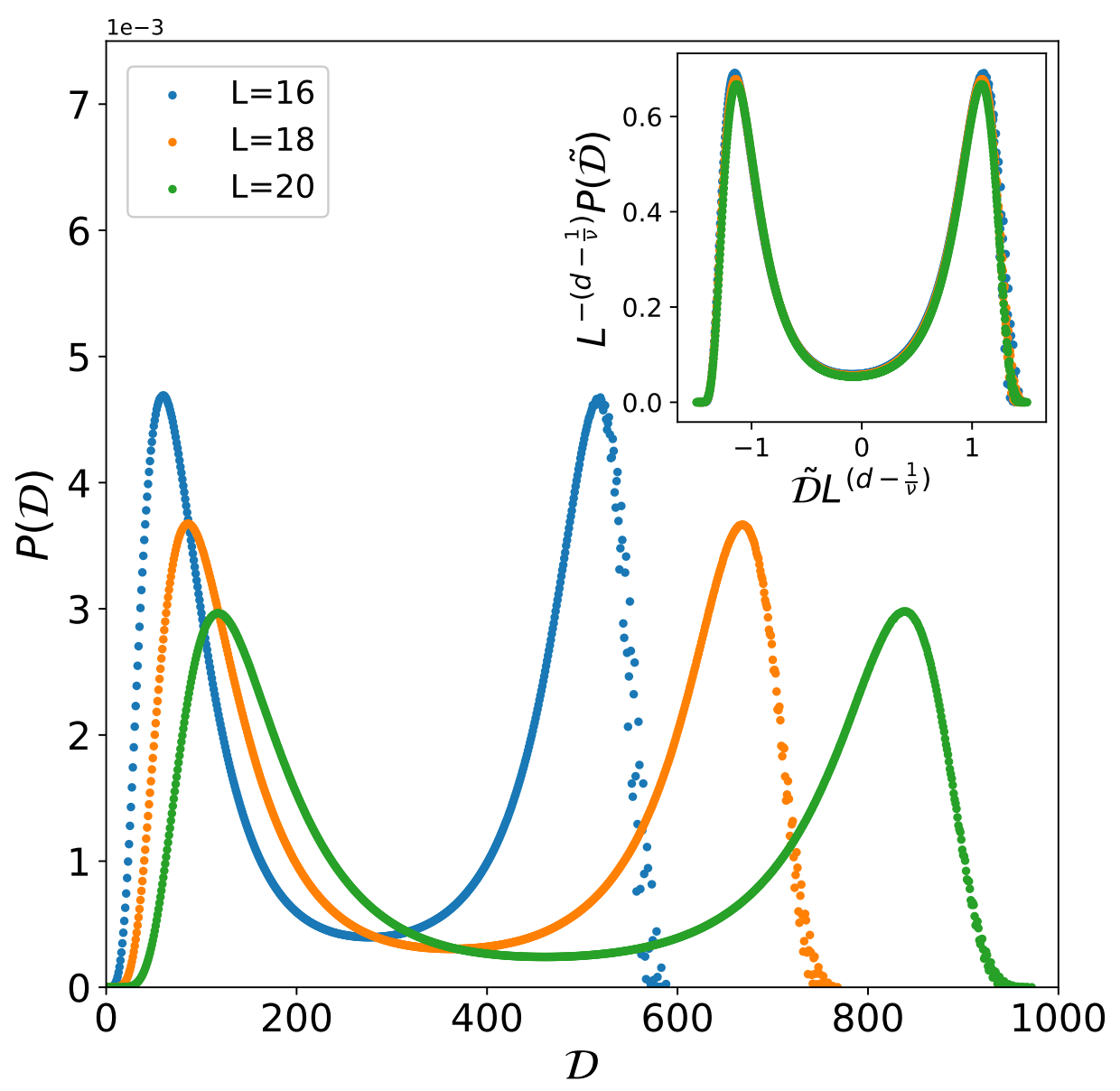

FigURA 4.7. Funciones de las distribuciones de las probabilidades de la variable $\mathscr{D}$ para $\alpha=1$, que muestra los dos picos para diferentes tamaños de red en el punto tricrítico, $T_{t c}=1,154(1)$. Las gráficas de escala de la función de distribución de probabilidad se muestran en el inset, con el exponente tricrítico $1 / v=1,86, d=2$ ya que estamos trabajando en una red bidimensional, y $D$ está normalizado para tener un promedio cero y una varianza unitaria.

\subsection{Fase Cuadrupolar}

La fase cuadrupolar es una fase ordenada que consiste en un arreglo de vacancias y espines a modo de tablero de ajedrez, tal y como se muestra en la figura(4.8).

$\mathrm{Al}$ estar rodeados de vacancias, los espines de los sitios ocupados, no tienen interacciones mutuas, por lo que el estado es perfectamente paramagnético. De esta manera, el 


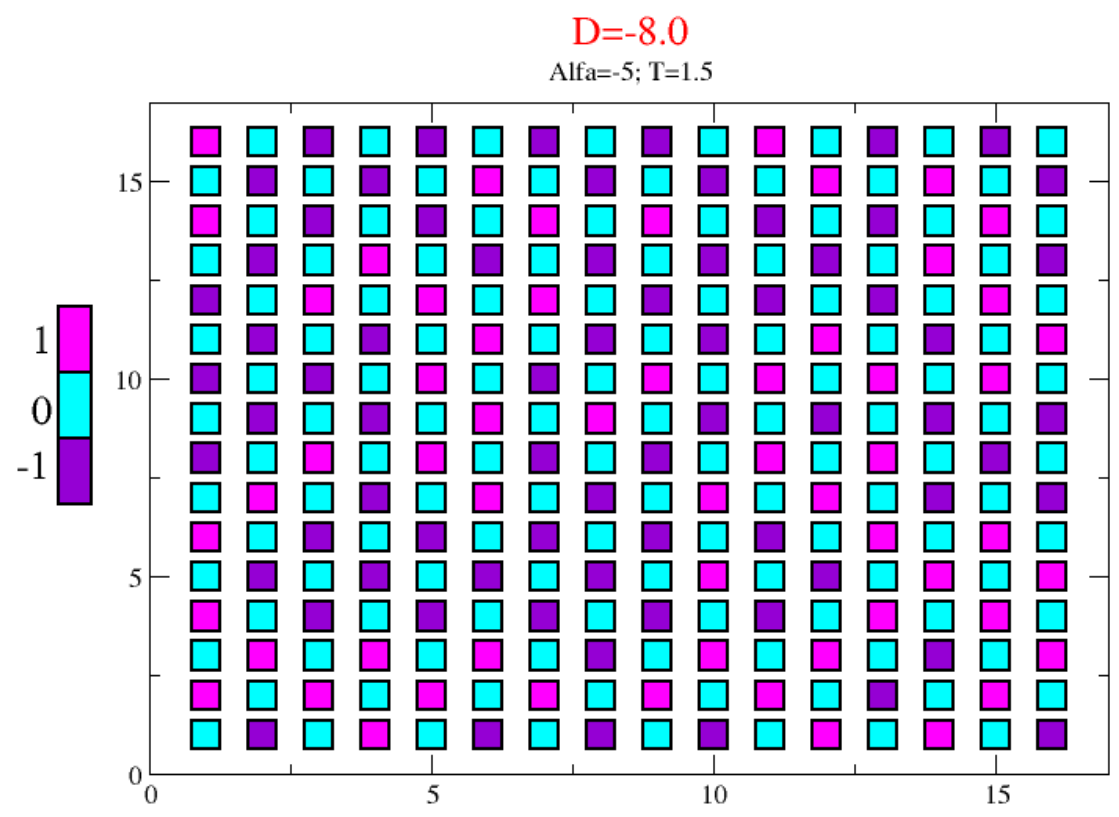

Figura 4.8. Modelo de BEG en una red cuadrada de $L=16$, en la fase cuadrupolar para $T=1,5, \alpha=5$ y $D=-8$. Los cuadrados magenta representan los espines positivos, los púrpuras los negativos y los turquesas las vacancias.

uso de la magnetización como parámetro de orden no es suficiente y habrá que buscar un nuevo parámetro de orden $(Q)$. Este está definido como

$$
Q=\frac{2}{N}\left(\sum_{f \in A} s_{f}^{2}-\sum_{g \in B} s_{g}^{2}\right),
$$

donde $N$ es el número total de sitios que tiene la red y las sumas sobre $A$ y $B$ corresponden a los sitios de las subredes.

En adelante, el estudio de las transiciones de fase en la fase cuadrupolar sigue, en líneas generales, el estudio de la sección 4.3. Los resultados principales se obtuvieron usando el algoritmo de Wang-Landau, pero se realizaron estudios de tamaño finito con el algoritmo de Metrópolis para comparar la precisión del método.

El panel superior de la figura(4.9) muestra el parámetro de orden $Q$ en función de la temperatura para muestras de distintos tamaños con $\alpha=-5$ y $D=-8$. El inset muestra 


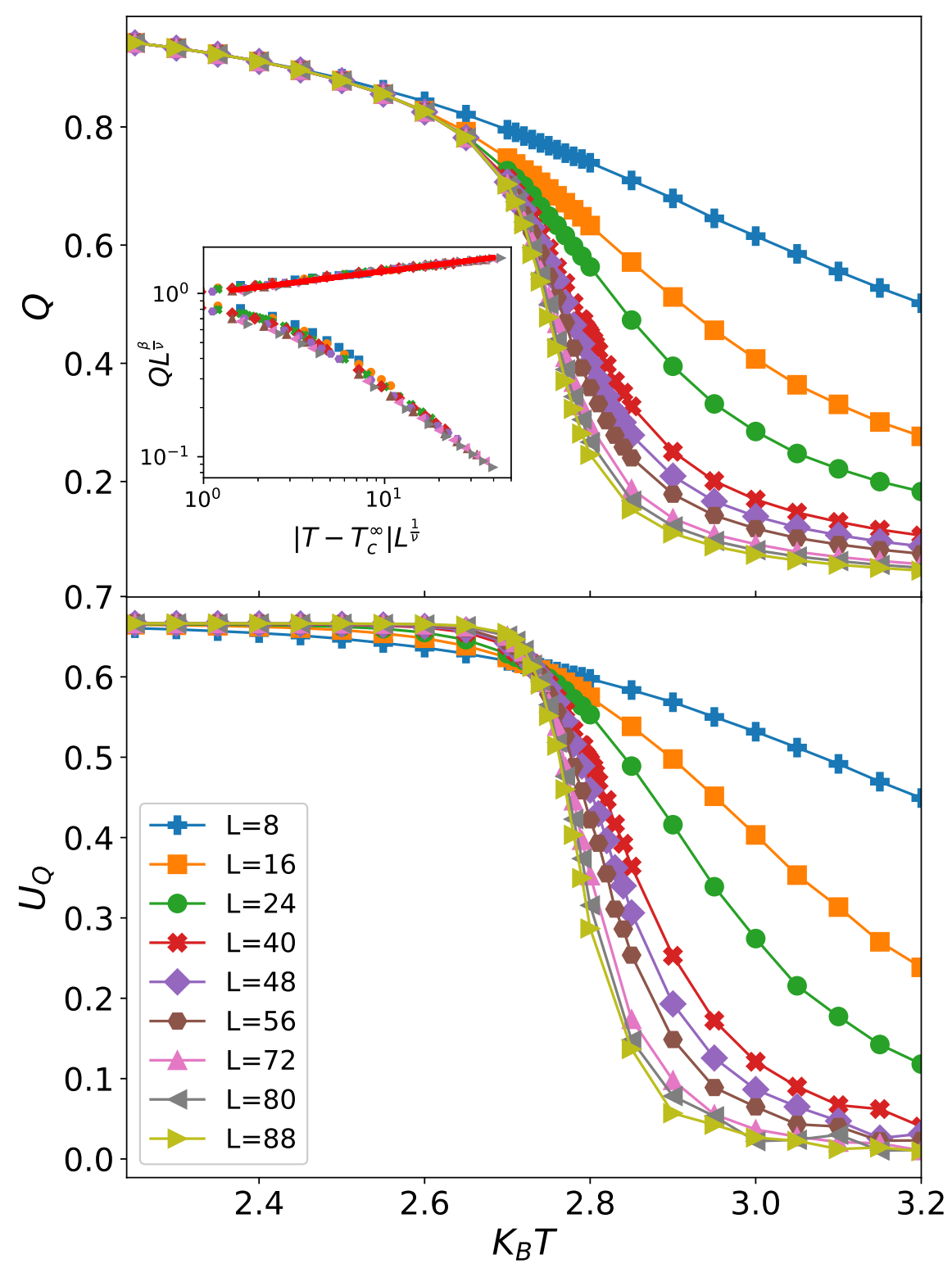

FIgURA 4.9. Panel superior: gráfico del momento cuadrupolar $v s$ la temperatura.

Se muestran los tamaños de la red y los parámetros son $\alpha=-5$ y $D=-8$. Inset: gráfico de escala de $Q L^{\beta / v}$ vs $\left|T-T_{c}^{\infty}\right| L^{1 / v}$, que muestra el colapso de los datos, obtenido al tomar $T_{c}^{\infty}=2,75(2)$. Si se ajustan estos datos, es posible recuperar $\beta$ de la pendiente de la rama $T>T_{c}^{\infty}$ (ver línea completa). Panel inferior: gráficos de $U_{Q}$ vs $k_{B} T$ obtenidos para redes de distintos tamaños y para la misma elección de $D$ y $\alpha$.

el colapso de los datos cuando se usan las variables reducidas $Q L^{\beta / v}$ y $\left|T-T_{c}\right| L^{1 / v}$. Con esto es posible chequear que la clase de universalidad corresponde a la de Ising para 
$2 D$, es decir, $v=1$ y $\beta=1 / 8$, y determinar la temperatura crítica $T_{c}^{\infty}=2,75(2)$. El panel inferior muestra el cumulante de $Q, U_{Q}$ en función de la temperatura para muestras de distintos tamaños y la misma elección de parámetros. No se observan transiciones de primer orden para $Q$.

Finalmente, para determinar el límite termodinámico de la temperatura crítica de la transición de segundo orden, se realiza un análisis de tamaño finito de la temperatura crítica efectiva.

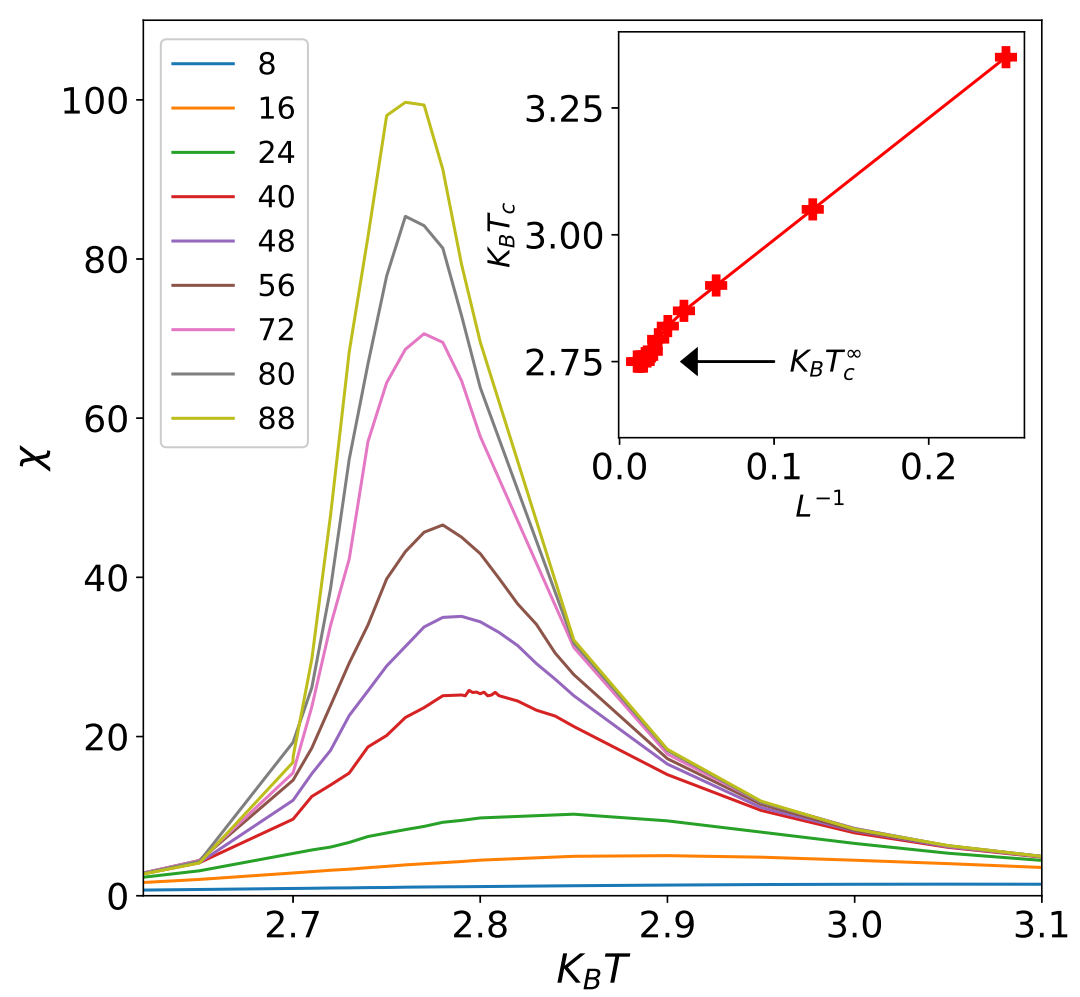

FiguRA 4.10. Gráfico de la dependencia de la temperatura crítica para sistemas de tamaño finito $T_{c}(L)$ determinado a partir de la posición de los picos de la susceptibilidad, para los parámetros $\alpha=-5$ y $D=-8$.

La figura(4.10) muestra un gráfico típico de la dependencia con respecto al tamaño de la temperatura crítica de tamaño finito $T_{c}(L)$ determinada a partir de la posición de los picos de la susceptibilidad. Usando la relación de escala

$$
T_{c}(L)=T_{c}^{\infty}+C L^{-1 / v},
$$


donde $T_{c}^{\infty}$ es la temperatura crítica en el límite termodinámico y se puede calcular como la extrapolación del gráfico de $T_{c}(L)$ vs $L$ (mostrado en el inset). De la extrapolación se obtiene que $T_{c}^{\infty}=2,75$ para esta elección particular de parámetros ( $\alpha=-5$ y $D=-8$ ), lo cual coincide con los resultados mostrados en figura(4.9).

Por lo tanto, este estudio basado en la combinación de los algoritmos WL y MU permitió determinar el diagrama de fase completo del modelo BEG. Los resultados obtenidos mediante el uso de ambos enfoques son totalmente coherentes entre ellos, así como con los cálculos exactos para el estado fundamental.

\subsection{Modelo de BEG con interacción a segundos vecinos}

El orden de tipo tablero de ajedrez que presenta la fase cuadrupolar figura(4.8), causa que los espines queden segregados por las vacancias. Por lo tanto, resulta interesante estudiar el comportamiento del sistema al agregar una interacción a segundos vecinos (NNN por sus siglas en inglés) para inducir el orden de los espines en la fase cuadrupolar. Esto es, si los primeros vecinos se encontraban a una distancia $l$, los segundos vecinos se encontrarán a una distancia $\sqrt{2} l$. La interacción NNN está caracterizada por la constante de acoplamiento $J_{2}$, la cual tiene el mismo signo que $J$ en (4.1). El nuevo Hamiltoniano va a tener la siguiente forma

$$
\mathscr{H}=\mathscr{H}_{B E G}-J_{2} \sum_{\langle\langle i, j\rangle\rangle} s_{i} s_{j}
$$

donde $\langle\langle i, j\rangle\rangle$ indica la suma sobre los segundos vecinos.

\subsubsection{Diagrama de fases para $T=0$}

$\mathrm{Al}$ igual que en la sección 4.2 es posible calcular el diagrama de fases $D$ vs $\alpha$ para $T=0$. 
- Para $D \rightarrow \infty$ (fase paramagnética)

$$
E \rightarrow 0
$$

- Para $D \rightarrow-\infty$ (fase ferromagnética)

$$
E \rightarrow-2 J+D-2 \alpha J-2 J_{2}
$$

- Para la fase cuadrupolar

$$
E \rightarrow \frac{D}{2}-\frac{2 J_{2}}{2}
$$

de modo que: la transición paramagnética $\leftrightarrow$ ferromagnética va a ocurrir a la largo de la línea

$$
\begin{aligned}
& 0=-2 J+D-2 J \alpha-2 J_{2} \\
& D=2 J(\alpha+1)+2 J_{2} .
\end{aligned}
$$

Para $\alpha<1$ se recupera la fase cuadrupolar que tiene lugar a lo largo de la línea

$$
\begin{aligned}
\frac{D}{2}-\frac{2 J_{2}}{2} & =-2 J+D-2 J \alpha-2 J_{2} \\
D & =4 J(\alpha+1)+2 J_{2},
\end{aligned}
$$

pero esta vez, la subred ocupada por los espines va a mostrar un orden ferromagnético para cualquier $J_{2}$ distinta de cero. Por tal motivo, se llamará a esta fase: cuadrupolar ferromagnética $\left(Q_{F}\right)$. En ella coexisten ambos tipos de orden: ferromagnético y cuadrupolar. Pero, aún para $T=0$ el sistema eventualmente se desordenará hacia una fase paramagnética, esto ocurrirá por encima de $D=2 J_{2}$. De éste análisis y de la figura(4.11) es posible observar que a medida que $J_{2}$ aumenta, la fase ferromagnética avanza sobre la paramagnética mientras que la fase cuadrupolar se mantiene constante. 


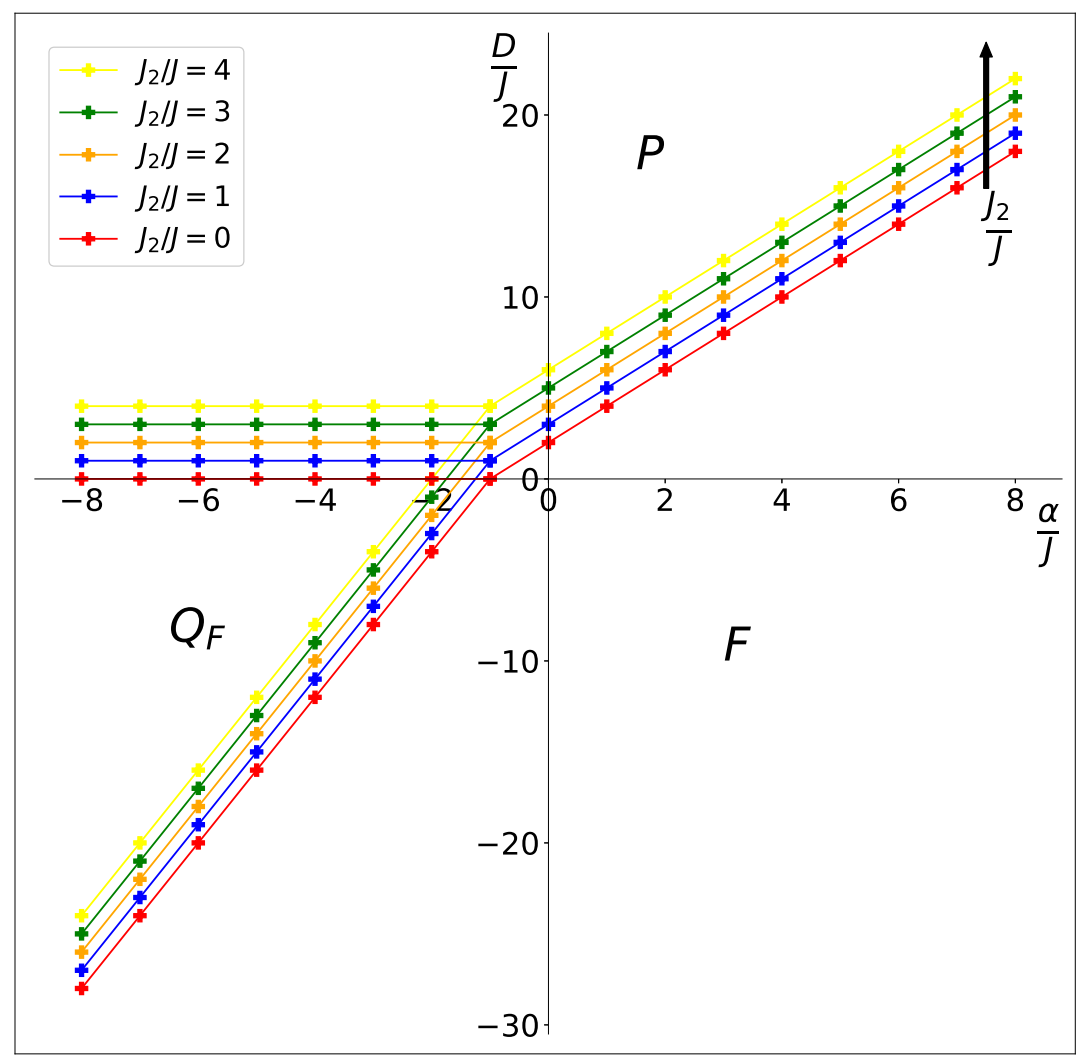

FIgURA 4.11. Diagrama de fases del modelo BEG con interacción NNN para $T=0$. Muestra el comportamiento de las tres fases: ferromagnética $(F)$, paramagnética $(P)$ y cuadrupolar-ferromagnética $\left(Q_{F}\right)$, a medida que aumenta $J_{2}$. Cuando $J_{2}=0$ se recupera el diagrama de fase BEG para $T=0$ que se muestra en la figura (4.2).

\subsubsection{Diagrama de fases para $T \neq 0$}

La figura(4.12) muestra el diagrama de fases, obtenido con el algoritmo de WL, en función de $D, J_{2}$ y $T$ para $\alpha=-5$. Es interesante destacar, que a medida que crece $J_{2}$, la fase cuadrupolar con orden ferromagnético, es decir la fase $Q_{F}$ encontrada para $T=0$, comienza a desarrollarse a temperaturas finitas, abarcando la fase cuadrupolar hasta que, por encima de $J_{2} / J$ entre 1 y 1,5 , todo el orden cuadrupolar muestra ferromagnetismo. La transición entre la fase cuadrupolar y la fase cuadrupolar con ferromagnetismo es de segundo orden, tal y como se muestra en la figura(4.13), y corresponde a la clase de 


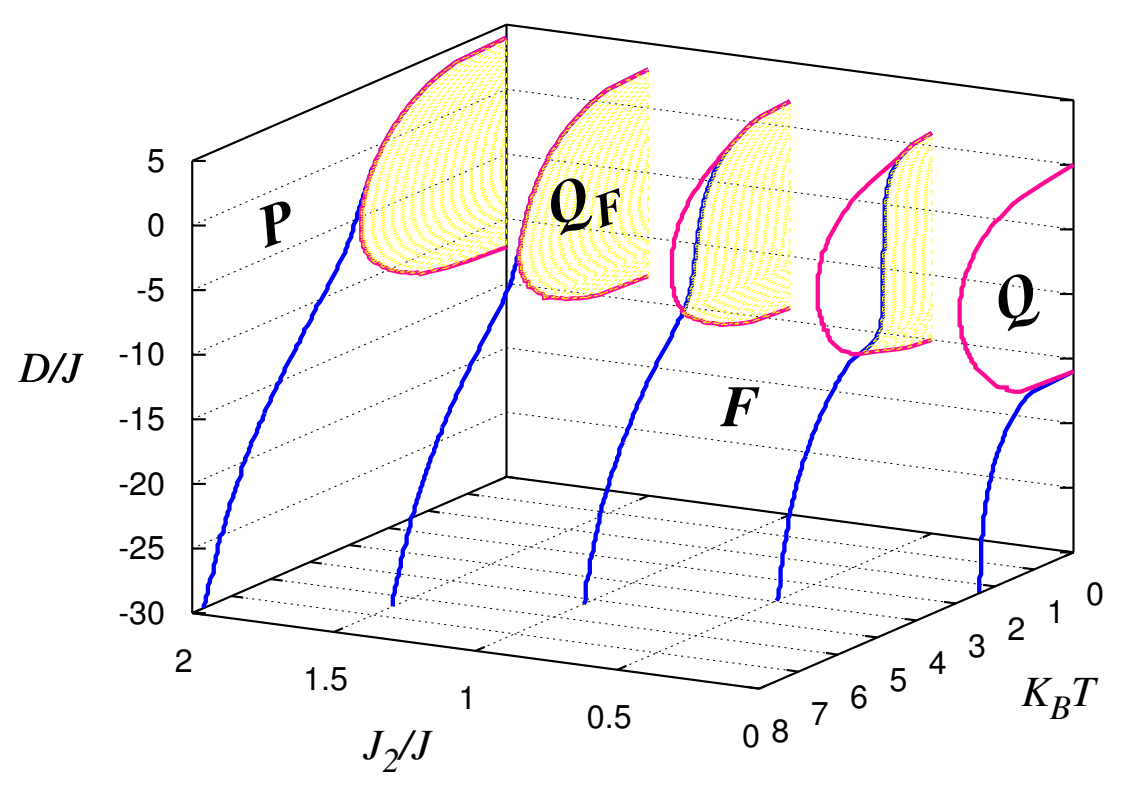

FIgURA 4.12. Diagrama de fases del modelo BEG con interacción NNN para $\alpha=-5$. Muestra el comportamiento de las cuatro fases: ferromagnética $(F)$ que se encuentra por debajo de la línea azul, paramagnética $(P)$, fuera de la línea azul, cuadrupolar $(Q)$, dentro de la línea rosa y cuadrupolarferromagnética $(Q F)$ la región amarilla dentro la línea rosa, a medida que $J_{2}$ aumenta. Cuando $J_{2}=0$ se recupera el diagrama de fase BEG que se muestra en la figura (5.2).

universalidad de Ising en 2D.

Análogamente a lo que se destacó en la ecuación (4.1), cuando $D \rightarrow-\infty$ las vacancias desaparecen, recuperando así el modelo de Ising con interacciones a segundos vecinos, por lo tanto las temperaturas críticas de las líneas de transición orden-desorden coinciden con las temperaturas exactas obtenidas de [82]:

$$
2 e^{-\frac{2 J}{k_{B} T_{c}}}\left(1-e^{-\frac{2 J}{k_{B} T_{c}}}\right)-e^{-\frac{2\left(J+J_{2}\right)}{k_{B} T_{c}}}-e^{-\frac{4 J_{2}}{k_{B} T_{c}}}=0
$$

Como se mencionó previamente, el tamaño de la red $L=16$ produce un desplazamiento en la temperatura crítica inferior al $6 \%$. 


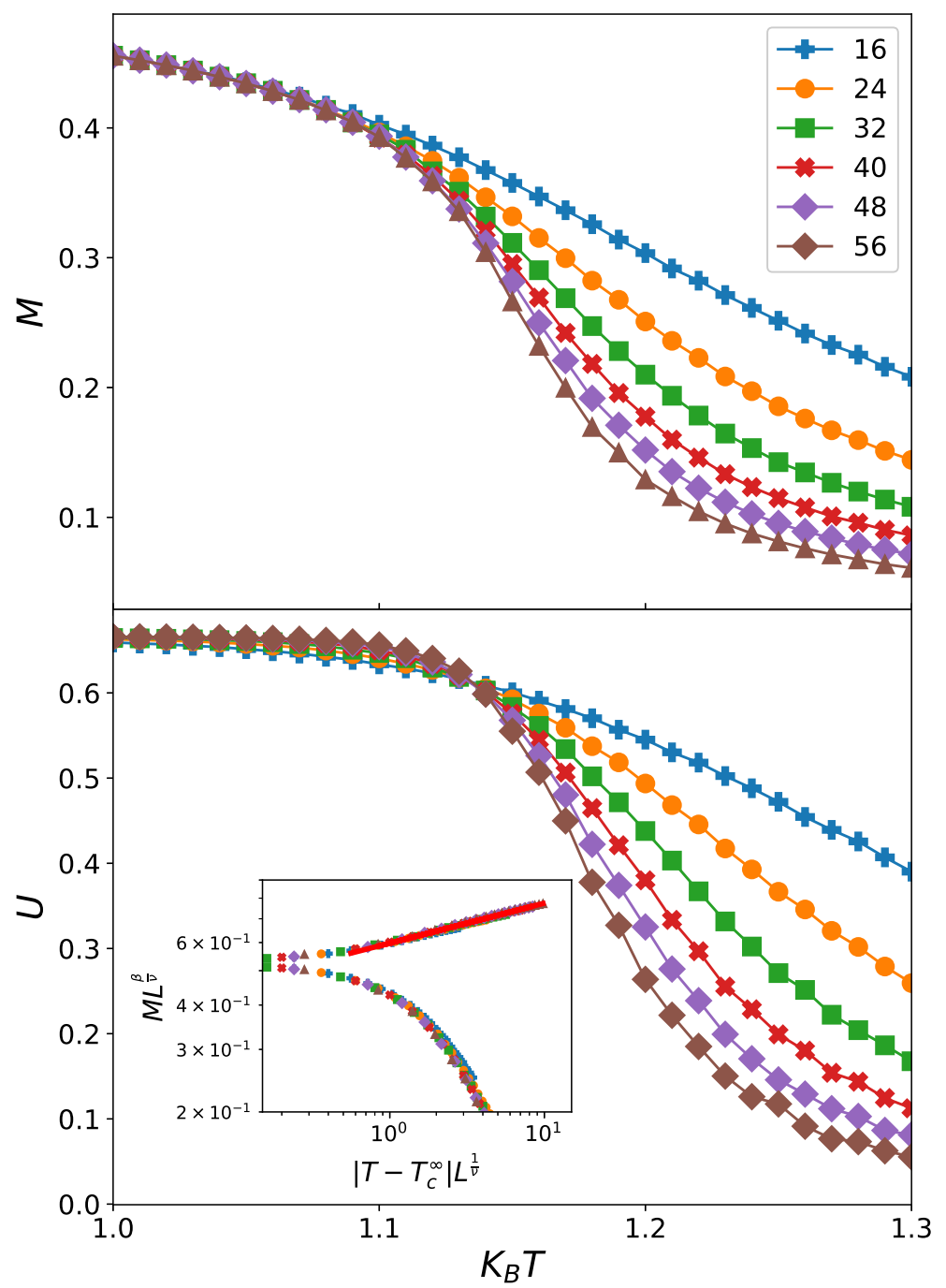

FIgURA 4.13. Panel superior: gráfico de la magnetización $\langle|m|\rangle v s$ la temperatura $k_{B} T$. Se muestran los tamaños de la redes y los parámetros son $J_{2}=0,5$, $\alpha=-5$ y $D=-8$. Inset: gráfico de escala de $M L^{\beta / v} v s\left|T-T_{c}^{\infty}\right| L^{1 / v}$, que muestra el colapso de los datos obtenido al tomar $v=1, \beta=1 / 8$ y $T_{c}^{\infty}=1,13(1)$. Si se ajustan los datos, es posible recuperar $\beta$ de la pendiente de la rama $T>T_{c}^{\infty}$ (ver línea completa). Panel inferior: gráficos de $U$ vs $k_{B} T$ obtenidos para muestras de distintos tamaños y para la misma elección de $D$ y $\alpha$, que muestran el punto de intersección común a la temperatura crítica dada por $T_{c}^{\infty} \simeq 1,13(1)$, en acuerdo con el valor utilizado para obtener colapso de datos (panel superior). 


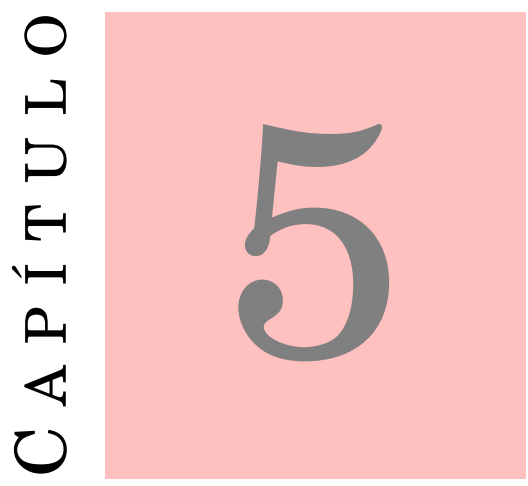

\section{ESTUDIO DEL EFECTO DEL}

\section{CONFINAMIENTO EN EL MODELO BEG}

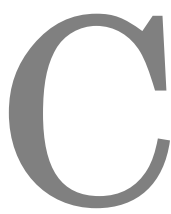

uando se habla de poner a un sistema bajo confinamiento, no sólo se hace referencia a la geometría del mismo, sino a los efectos que esta conlleva, como ser aquellos causados por el tamaño finito, o por la superficie, etc. Estos provocan grandes cambios en el comportamiento del sistema. Por ejemplo, como se ve en la figura(5.1), la condensación de gas-líquido se ve fuertemente afectada por la presencia de paredes atractivas o repulsivas.

Un gas saturado en una geometría semi-infinita expuesta a una pared atractiva puede mostrar una transición de mojado [83], es decir, el espesor de una capa líquida adsorbida en la pared aumenta desde unas pocas separaciones interatómicas hasta un valor macroscópico. De forma análoga, para una pared repulsiva, puede ocurrir una 

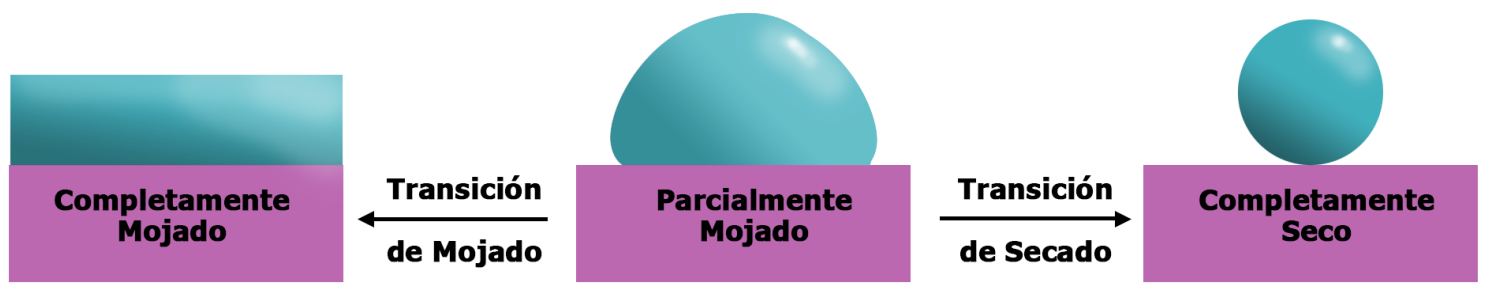

FIGURA 5.1. Representación esquemática de las trasiciones de mojado y secado.

transición de secado (el papel de las fases líquida y gaseosa del fluido se intercambia).

Esta situación se ve modificada cuando se confina un fluido entre dos paredes separadas por una distancia $L$, ya que los efectos superficiales, como los fenómenos de mojado o secado competirán con los efectos de tamaño finito.

Una de las interpretaciones que recibe la transición de mojado, la cual se utilizará en este trabajo, es considerarla como una transición de deslocalización de la interfase.

En esta interpretación, las fases gas-líquido están representadas por espines up (1) y down (-1). Asumiendo que una de estas fases de espines coexistentes en el bulk (para el campo magnético de bulk $h_{b}=0$ ) es preferida por las paredes, es decir, las paredes son atractivas, es natural introducir el concepto de campos magnéticos superficiales $h_{s}$ ( $h_{1}=h_{L}$ en las superficies 1 y $L$ respectivamente), los cuales actúan sobre los espines que se encuentran en contacto con dichas paredes. La variación resultante de la coexistencia de fases en el bulk $\left(h_{b}=0\right)$, ocurre ahora para un valor $h_{\text {coex }}$ distinto de cero, esto es el análogo de la condensación capilar o evaporación en fluidos. Si los campos magnéticos superficiales fueran antisimétricos $\left(h_{1}=-h_{L}\right)$, la coexistencia de fases vuelve a ocurrir para $h_{b}=0$, pero aparte de la transición en la que el sistema pasa de una fase en la cual predominan los espines up, a otra fase en la que predominan los espines down, ocurre la transición de deslocalización de la interfase. Cuando la interfase está fuertemente unida a la pared, es decir, predomina una especie de espines, se dice que el sistema se 
encuentra en un estado no-mojado, mientras que en el estado mojado la interfase fluctúa libremente lejos de la pared (coexisten las dos especies de espines).

En este capítulo se estudiarán los efectos del confinamiento sobre el modelo BEG desarrollado en el capítulo anterior, mediante técnicas ampliamente utilizadas en el campo de la Mecánica Estadística, haciendo especial hincapié en la comprensión y la caracterización de fenómenos como las transiciones de mojado y la adsorción interfacial, prestándole particular atención al comportamiento que presentan las vacancias.

Hasta ahora, todos los estudios que se llevaron a cabo, se basaron en sistemas cuyas longitudes lineales eran iguales. En otras palabras, se trabajó con geometrías cuadradas en redes cuadradas. En las siguientes secciones se buscará generalizar dichos estudios para sistemas que presenten dos longitudes lineales distintas $L_{\|}$y $L_{\perp}$, es decir, geometrías rectangulares en redes cuadradas.

\subsection{El modelo}

Se considera el Hamiltoniano del modelo de BEG (4.1) en una red cuadrada de geometría $L \times M$. Dicha red presenta condiciones periódicas de contorno en la dirección $x$ (donde la red tiene una longitud $M$ ) y condiciones de contorno libres (paredes) en la dirección $y$, sobre las cuales actúan campos superficiales $h_{1}$ y $h_{L}$. Se puede ver una representación esquemática en la figura(5.2).

El Hamiltoniano resultante tiene la siguiente forma:

$$
\mathscr{H}=-J \sum_{\langle i, j\rangle} s_{i} s_{j}+D \sum_{i=1}^{N} s_{i}^{2}-\alpha J \sum_{\langle i, j\rangle} s_{i}^{2} s_{j}^{2}-h_{1} \sum_{i \in 1} s_{i}-h_{L} \sum_{i \in L} s_{i}
$$

La constante de acoplamiento $J$ es homogénea a lo largo de todo el sistema, por lo que no se considera la posibilidad de sumar una constante $J_{S}$ diferente para los espines del borde como se ha hecho en otros estudios [7, 13, 14, 17-19, 25]. Se tomará la separación de la red como unidad de longitud, por lo que el número total de espines no es más que el 


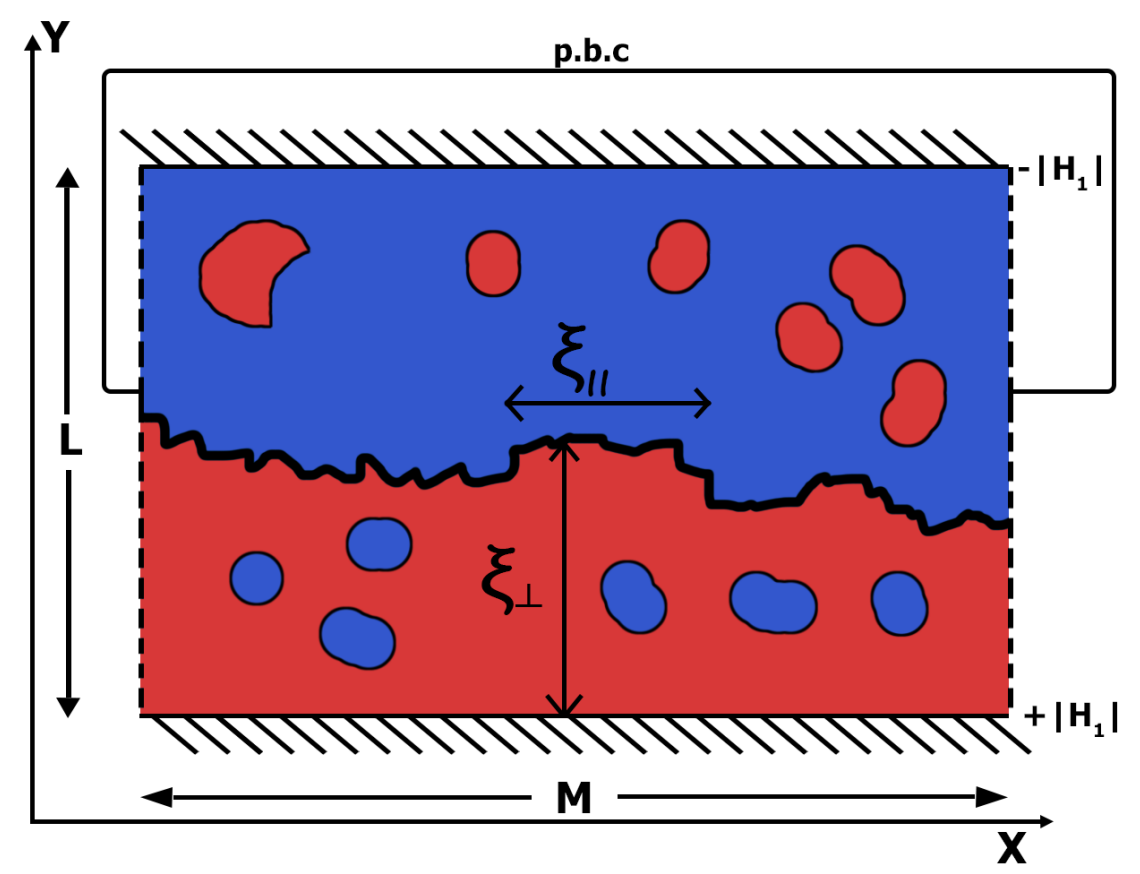

FiguRA 5.2. Descripción esquemática de la geometría del sistema y su estado en la temperatura de mojado $T_{w}\left(h_{1}\right)$. En dicho estado, la interfase se ha separado de la pared inferior y se encuentra fluctuando en el medio de la red. Esta separa dos dominios con magnetizaciones opuestas, donde el dominio positivo es representado con rojo y el negativo con azul. Se indica la elección de las longitudes lineales $L$ y $M$, y de las condiciones de contorno: periódicas en la dirección $x$ y libres en la dirección $y$. Sobre esta última actúan los campos superficiales $h_{1}$ y $h_{L}=-h_{1}$. Se muestran también las longitudes de correlación: paralela $\xi_{\|}$y perpendicular $\xi_{\perp}$ a la interfase. Estas cumplen $\xi_{\|} \propto \xi_{\perp}^{2}$, para casos particulares como el modelo de Ising, el BC y el BEG, no así para el modelo RBIM.

producto de las longitudes lineales del sistema, $N=L \times M$. Los campos $h_{1}$ y $h_{L}$ actúan solamente sobre los espines de la primera y última fila respectivamente. Estos serán homogéneos o heterogéneos dependiendo del tipo de estudio que se realice. En ambos casos, se trabajará en la fase ferromagnética del diagrama de fases de la figura(4.2) y las transiciones ocurrirán a temperaturas menores a la $T_{c b}$. 


\subsection{Wetting crítico en dos dimensiones}

Si se consideran sistemas semi-infinitos con longitudes $L \rightarrow \infty$ y $M \rightarrow \infty$, sobre los cuales actúan campos superficiales inhomogéneos, es decir $h_{1}=-h_{L}<0$, el sistema presentará dos transiciones de fase. En el límite termodinámico a temperatura $T_{c b}\left(D_{c b}\right)$ la transición ocurre de una fase desordenada paramagnética a una fase ordenada ferromagnética, tal y como se vio en el capítulo 4 . Por otro lado, a una temperatura $T_{w}\left(h_{1}\right)<T_{c b}\left(D_{c b}\right)$ ocurre una segunda transición en la cual la interfase generada por los campos se desprende de la pared y fluctúa libremente en el medio de la red. Este fenómeno, asociado a una interfase entre espines en un sistema magnético, es formalmente equivalente a la transición de mojado que puede experimentar un liquido o fluido en contacto con una superficie, tal como se discutió dentro del contexto de la figura (5.1). En este caso se dice que el sistema pasa de un estado no-mojado a otro mojado, por lo que dicha transición se conoce como transición de mojado.

Para sistemas semi-infinitos, la transición de mojado representa una singularidad en el exceso de energía libre superficial $f_{s}^{(1)}\left(T, h_{1}\right)$, definido a partir de la descomposición de la energía libre total, en un término correspondiente al Bulk y dos términos correspondientes a los bordes para $L \rightarrow \infty$ y $M \rightarrow \infty$

$$
\frac{1}{L M} f\left(T, h_{1}, h_{L}, L, M\right)=f_{b}(T)+\frac{1}{L} f_{s}^{(1)}\left(T, h_{1}\right)+\frac{1}{L} f_{s}^{(L)}\left(T, h_{L}\right)
$$

donde las variables $D$ y $\alpha$ son constantes y no se presentan explícitamente en la ecuación con el fin de simplificar la notación. La parte singular de la energía libre con condiciones de contorno libres se conoce comotensión superficial o exceso de energía libre superficial, y se espera que cerca de la $T_{w}\left(h_{1}\right)$, satisfaga

$$
\frac{1}{k_{B} T} f_{s, s i n g}^{1}\left(t, h_{1}\right)=|t|^{2-\alpha_{s}} \tilde{F}_{s}\left(h_{1}|t|^{-\Delta_{s}}\right)
$$

donde $t=\left|T-T_{w}\left(h_{1}\right)\right| \rightarrow 0$ y $\tilde{F}_{s}$ es una función de escala. Además $\alpha_{s} \mathrm{y} \Delta_{s}$ son exponentes 
críticos que caracterizan el comportamiento singular de la transición de mojado crítica en dos dimensiones debido al exceso superficial.

Como se mencionó previamente, cuando se consideran fenómenos en las proximidades del punto crítico, la longitud de correlación diverge. Ya que una de las formas de tratar la transición de mojado es mediante una transición de deslocalización de la interfase, se pueden estudiar las fluctuaciones de la altura del contorno de dicha interfase $\ell(x)$, la cual separa el dominio con magnetización negativa de aquel con magnetización positiva, mediante la función de correlación $G(x)$. Por lo tanto, definiendo $\delta \ell(x)=\ell(x)-\langle\ell\rangle$ se tiene que

$$
G(x)=\langle\delta \ell(0) \delta \ell(x)\rangle_{T}
$$

donde $\langle\cdots\rangle_{T}$ denota el promedio en el colectivo canónico a temperatura $T$. Cerca del punto crítico, $G(x)$ presenta un comportamiento análogo al decaimiento de las correlaciones del bulk (3.4), por lo tanto escalea como

$$
G(x) \sim x^{-\left((d-1)-2+\eta_{\|}\right)} G\left(\frac{x}{\xi_{\|}}\right) .
$$

Es importante hacer una aclaración antes de seguir con el desarrollo de escala de la transición de mojado. En el análisis realizado para sistemas geométricamente simétricos en el capítulo 3 , las transiciones de fases continuas se caracterizaban mediante una longitud de correlación $\xi$, la cual divergía en la temperatura crítica como $\left|T-T_{c b}\right|^{-v}$. Para el caso de fenómenos críticos anisotrópicos como el que se está tratando ahora, la función de correlación decae con longitudes de correlación, $\xi_{\|}$y $\xi_{\perp}$ según la dirección de la red, donde los índices $\| \mathrm{y} \perp$ representan las longitudes paralela y perpendicular a la interfase. Además en las proximidades del punto crítico, dichas longitudes divergen con exponentes críticos distintos, $v_{\|} \mathrm{y} v_{\perp}$ respectivamente. Por lo tanto, en el régimen de escala, ambas longitudes de correlación se relacionan mediante $\xi_{\|} \sim \xi_{\perp}^{z}$ donde $z=v_{\|} / v_{\perp}$ es el exponente dinámico, que dice qué tan rápido se dispersa la perturbación local. Al igual que los otros 
exponentes, $z$ es una cantidad universal, es decir, su valor es determinado mediante la clase de universalidad y la dimensión del sistema.

Retomando la ecuación (5.5), $\eta_{\|}$(análogo a $\eta$ en el bulk) es el exponente que describe la variación de la correlación a grandes distancias en el wetting crítico (en la criticalidad del bulk) donde $\xi_{\|}(\xi)$ es infinita. Para $\xi_{\|}$existe una relación análoga a la de la ecuación(5.3), inspirada en el comportamiento del bulk, ecuación(3.1d)

$$
\xi_{\|}=t^{-v_{\|}} \tilde{\xi}_{\|}\left(t^{-\Delta_{s}}\right)
$$

Según las relaciones de hiper escala citadas en el capítulo 3 , particularmente $2 \beta+\gamma=$ $2-\alpha=v d$ y $\gamma=(2-\eta) v$, se pueden expresar todo los exponentes críticos en términos de dos exponentes independientes $\eta$ y $v$. Esto se mantiene para las transiciones de mojado, donde

$$
2 \beta_{s}+\gamma_{s}=2-\alpha_{s}=(d-1) v_{\|} \quad \text { y } \quad \gamma_{s}=\left(2-\eta_{\|}\right) v_{\|} .
$$

Conforme a la teoría de ondas capilares [83] cerca del punto crítico $\left(\xi_{\|} \rightarrow \infty\right), G$ escalea como $G(x, \infty) \sim x^{-(d-3)}$ para $d \leq 3$, por lo tanto $\eta_{\|}=0$. De esta manera, hay un solo exponente crítico independiente [83]

$$
v_{\|}=2
$$

y las relaciones de hiper escala (5.7) para fenómenos interfaciales con $d=2$ implican que $\alpha_{s}=0$. Por lo tanto, de acuerdo con la ecuación (5.5), se puede concluir que en una longitud de escala $x$, la interfase muestra (a $t=0$ ) un desplazamiento cuadrático medio, también proporcional a $x$. Mientras que para $t>0$, en la longitud de escala $\xi_{\|}$, dicho desplazamiento ocurre en la dirección $y$ y es proporcional a $\xi_{\|}$. Por último, cuando $x \gg \xi_{\|}$, la correlación $G(x) \rightarrow 0$, por lo tanto la longitud de correlación $\xi_{\perp}$ escalea como

$$
\xi_{\perp}^{2} \propto \xi_{\|}
$$


De forma análoga a la ecuación (5.6) para $\xi_{\|}$, se tiene $\xi_{\perp} \sim t^{-v_{\perp}}$ por lo tanto $v_{\perp}=1$. Con este resultado y teniendo en cuenta que los excesos superficiales de magnetización y susceptibilidad se pueden obtener derivando la energía libre, se concluye que dichas cantidades tienen singularidades:

$$
\begin{array}{lcccr}
m_{s} \propto t^{2-\alpha_{s}-\Delta_{s}} & \text { pero } & m_{s} \propto t^{\beta_{s}} & \text { entonces } & 2-\alpha_{s}-\Delta_{s}=\beta_{s} \\
\chi_{s} \propto t^{2-\alpha_{s}-2 \Delta_{s}} & \text { pero } & \chi_{s} \propto t^{-\gamma_{s}} & \text { entonces } & 2-\alpha_{s}-2 \Delta_{s}=-\gamma_{s}
\end{array}
$$

Usando la consideración de que este exceso de susceptibilidad es provocado por los desplazamientos que experimenta la interfase, se puede deducir la relación de escala entre $\Delta_{s}, v_{\|} \mathrm{y} \eta_{\|}$a partir de la ecuación (5.5)

$$
\Delta_{s}=\frac{v_{\|}}{2}\left[-(d-1)+2-\eta_{\|}\right]
$$

para $d=2$ у $\eta_{\|}=0, \Delta_{s}=3$.

Dicho razonamiento se puede aplicar a la magnetización, en la que, por las ecuaciones (5.10) resulta

$$
m_{s} \propto\langle\ell\rangle^{2-\alpha_{s}-\Delta_{s}}=\langle\ell\rangle^{-1} \propto \xi_{\perp}
$$

por lo tanto, cerca del wetting crítico en $\mathrm{d}=2$, la distancia media crítica de la interfase con respecto a la pared, y la variación de la misma con respecto a su media, son del mismo orden

$$
\beta_{s}=v_{\perp}=1
$$

Si bien este enfoque es completamente natural en un contexto analítico y los exponentes críticos permiten describir el comportamiento del wetting crítico en dos dimensiones, no es útil al momento de llevar a cabo simulaciones computacionales. Por tal motivo, se realizará un estudio de escala para tamaño finito, propuesto por Albano y Binder [27], el cual consiste en describir las transiciones de wetting mediante un nuevo parámetro de orden. En este caso, dicho parámetro será la magnetización total del sistema, o estrictamente hablando, su valor absoluto, el cual pasa de cero a un valor distinto de cero al 
cruzar la temperatura crítica. De esta manera, la transición de wetting es equivalente a un fenómeno crítico de "bulk" con el exponente del parámetro de orden igual a cero.

Partiendo de la ecuación (3.22) presentada en el capítulo 3, la cual muestra la función de distribución de la probabilidad de la magnetización total para sistemas finitos isotrópicos, con dimensión lineal $L$ en todas sus direcciones. Es posible llevar a cabo una generalización para sistemas con dimensiones lineales anisotrópicas $L$ y $M$, y exponentes de longitud también anisotrópicos $v_{\|}$y $v_{\perp}$ como los considerados en este capítulo. Dicha FDP tiene la forma

$$
P_{L, M}(m)=\xi_{\|}^{\beta / v_{\|}} \tilde{P}\left(\frac{L^{v_{\|} / v_{\perp}}}{M}, \frac{M}{\xi_{\|}}, m \xi_{\|}^{\beta / v_{\|}}\right) .
$$

Mientras que para fenómenos críticos isotrópicos un sistema con geometría anisotrópica daría lugar a una dependencia simplemente sobre la relación de aspecto $L / M$ del sistema, el hecho de que $L$ escalee $\operatorname{con} \xi_{\|}$y $M$ con $\xi_{\perp}$, se puede usar para demostrar que en la ecuación (5.15) la dependencia de $L$ se da por la relación de aspecto generalizada $L^{z} / M$, donde $z=v_{\|} / v_{\perp}$ es el exponente dinámico que, considerando los resultados anteriores, $z=2 / 1=2$. Esto implica que la relación $L^{2} / M$ tiene que mantenerse constante cuando se estudie la variación con respecto a $M$.

El prefactor $\xi_{\|}^{\beta / v_{\|}}$de la ecuación (5.15), da la pauta de que la FDP puede ser normalizada. Además, procediendo de forma análoga al desarrollo para sistemas semi-infinitos, si se toman los momentos adecuados de $P_{L, M}(m)$, se obtiene

$$
\begin{gathered}
\langle|m|\rangle=\xi_{\|}^{-\beta / v_{\|}} \tilde{m}\left(\frac{L^{v_{\|} / v_{\perp}}}{M}, \frac{M}{\xi_{\|}}\right), \\
\left\langle m^{2 k}\right\rangle=\xi_{\|}^{-2 k \beta / v_{\|}} \tilde{m}_{2 k}\left(\frac{L^{v_{\|} / v_{\perp}}}{M}, \frac{M}{\xi_{\|}}\right),
\end{gathered}
$$

donde $k=1,2, \ldots$ A partir de (5.16) y (5.17) se puede obtener el comportamiento estándar de la susceptibilidad

$$
k_{B} T \chi=L M\left(\left\langle m^{2}\right\rangle-\langle|m|\rangle^{2}\right)
$$


y por lo tanto

$$
k_{B} T \chi=L M \xi_{\|}^{-2 \beta / v_{\|}} \tilde{\chi}\left(\frac{L^{v_{\|} / v_{\perp}}}{M}, \frac{M}{\xi_{\|}}\right) .
$$

La tarea fundamental ahora es identificar el exponente crítico del parámetro de orden, $\beta$. Para ello hay que notar dos cosas: en primer lugar, el comportamiento singular de $\chi$ sólo se puede deber al comportamiento singular de $\chi_{s}$, el cual, según la ecuación (5.10) y, usando los valores encontrados previamente $\alpha_{s}=0$ y $\Delta_{s}=3$, resulta $\chi_{s} \propto t^{-4}$. En segundo lugar es importante destacar que $\chi$ está normalizada por espín, es decir, por el volumen total $L \times M$, mientras que $\chi_{s}$ sólo considera los espines del borde, por lo que normaliza sólo con $M$. Por lo tanto en la $T_{w}$, para eliminar $L$ de la ecuación (5.19) hay que tomar $L^{2} / M=c$, de modo que $L=\sqrt{M c}$ y así

$$
k_{B} T \chi \propto \sqrt{c} M^{3 / 2-2 \beta / v_{\|}}
$$

independientemente del valor que tome la constante $c$. Por lo tanto, escribiendo

$$
\left.k_{B} T \chi\right|_{T_{w}}=\frac{1}{L} k_{B} T \chi_{s}=k_{B} T \chi_{s} M^{-1 / 2} c^{-1 / 2},
$$

y usando la relación de escala para tamaño finito de $\chi_{s}$,

$$
\chi_{s}=t^{-4} \tilde{\chi}\left(\frac{M}{\xi_{\|}}\right) \propto \xi_{\|}^{2} \tilde{\chi}\left(\frac{M}{\xi_{\|}}\right) \propto M^{2},
$$

para $T=T_{w}$. De esta manera

$$
\left.k_{B} T \chi\right|_{T_{w}} \propto M^{3 / 2}\left(\propto L^{3}\right)
$$

Comparando las ecuaciones (5.20) y (5.23) se obtiene

$$
\beta=0,
$$

e, interpretando la ecuación (5.23) en términos de los reultados estandares del escaleo de tamaño finito,

$$
\left.k_{B} T \chi\right|_{T_{w}} \propto M^{\gamma / v_{\|}}, \quad \gamma=3
$$


Es importante destacar que estos exponentes críticos satisfacen no sólo la relación de escala con el exponente de gap,

$$
\gamma+\beta=\Delta_{s}=3
$$

sino que también satisfacen la relación de hiperescala para $d=2$

$$
v_{\|}+v_{\perp}=2 \beta+\gamma=3
$$

Finalmente, los valores de los exponentes críticos obtenidos hasta ahora se muestran en la tabla (5.1)

\begin{tabular}{||l|l|l|l|l|l|l||}
\hline \hline \multicolumn{7}{||c|}{ Exponentes críticos BEG bajo confinamiento } \\
\hline \hline$\alpha_{s}$ & $\beta_{s}$ & $\gamma_{s}$ & $\Delta_{s}$ & $\eta_{s}$ & $v_{\|}$ & $v_{\perp}$ \\
\hline 0 & -1 & 4 & 3 & 0 & & \\
\hline$\alpha$ & $\beta$ & $\gamma$ & $\Delta$ & $\eta$ & 2 & 1 \\
\hline-1 & 0 & 3 & 3 & 0 & & \\
\hline
\end{tabular}

Tabla 5.1: Exponentes críticos del modelo BEG sometido al confinamiento. Arriba se muestran aquellos obtenidos a partir de exceso superficial, es decir, en las proximidades de la superficie bajo la acción de campos superficiales. Estos permiten describir el comportamiento del wetting crítico analíticamente, pero no son útiles para describir los resultados de las simulaciones computacionales. Para ello se propone el uso de un nuevo parámetro de orden y mediante la teoría de escala se obtienen los exponentes críticos(abajo). Ambos están relacionados entre sí y comparten $v_{\perp}$ y $v_{\perp}$. Más detalles en el texto. No confundir los exponentes del nuevo parámetro de orden con aquellos que describen el comportamiento crítico del Bulk, los cuales corresponden a la clase de universalidad de Ising.

Estos exponentes se han probado válidos para distintos modelos, tales como el modelo de Ising y el de Blume-Capel [27, 84, 85]. A continuación se mostrará que el uso de la magnetización total del sistema como parámetro de orden con exponente crítico $\beta=0$, también sirve para describir los resultados de las simulaciones del wetting crítico en el modelo BEG. 


\subsubsection{Resultados Monte Carlo}

En la mayoría de los estudios numéricos que se presentan en este capítulo, se ha utilizado un valor particular de la relación de aspecto generalizado:

$$
\frac{L^{2}}{M}=c=\frac{9}{8}
$$

El valor de la constante $c$, en principio, es arbitrario y los resultados sobre la ubicación de $T_{w}\left(H_{1}\right)$ y los exponentes críticos no deben depender de esta elección. Por lo que dicho valor fue elegido simplemente porque genera soluciones enteras y pequeñas para $L$ y $M$ : $(L, M)=(6,32) ;(12,128) ;(18,288) ;(24,512) ;(30,800) ;(36,1152) ;(48,2048)$. El efecto de considerar otras opciones de $c$ se discute en [27].

Las simulaciones de Monte Carlo se realizaron utilizando el algoritmo de Metrópolis. Las ejecuciones se realizan en una longitud de $10^{7}$ pasos de Monte Carlo por sitio red (MCS), sin tener en cuenta los primeros $2 \times 10^{6} \mathrm{MCS}$, que se usan para permitir que el sistema alcance el equilibrio.

Se analizaron los dos primeros momentos de la magnetización $\langle|m|\rangle,\left\langle m^{2}\right\rangle$ y el cumulante de cuarto orden de Binder (4.8), los cuales se graficaron en función de la temperatura. La precisión es mejor en los momentos de las magnetizaciones que en el cumulante.

Para transiciones de fase estándar, por ejemplo figura(4.3), las magnetizaciones no mostrarán puntos de intersección independientes del tamaño, tal que la intersección en la figura (5.3) es una clara evidencia del escaleo propuesto en la sección anterior. Por otro lado, los snapshots de las configuraciones del sistema a distintas temperaturas (Fig.5.4) son compatibles con el esquema cualitativo mostrado en la Figura(5.2).

En la fase mojada, la posición promedio de la interfase se encuentra en el medio de la red, entre las filas $y=L / 2$ y $y=1+L / 2$, para $L$ par; pero debido a las ondas capilares en la escala $M$ en la dirección $x$, la interfase realiza excursiones de orden $\sqrt{M}$ en la 


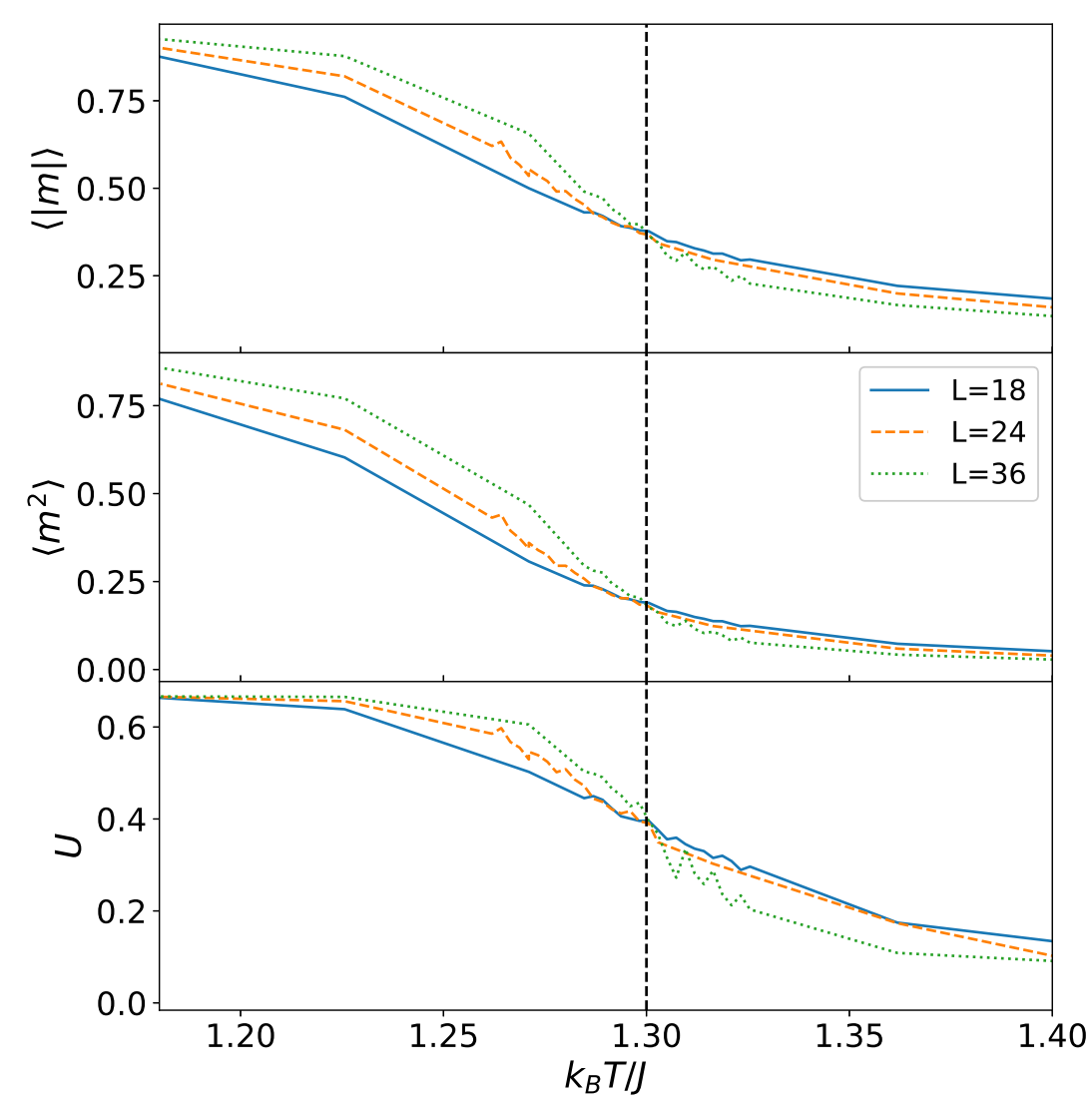

FiguRA 5.3. Gráficos del valor absoluto promedio de la magnetización $\langle|m|\rangle$ (arriba), del segundo momento de la magnetización $\left\langle m^{2}\right\rangle$ (centro) y del cumulante de cuarto orden de Binder $U$ (abajo) $v s$ la temperatura $k_{B} T$, para la elección $\alpha=1, D=3$ y $h_{1}=0,7$. Las líneas verticales indican la ubicación del punto de intersección independiente del tamaño, el cual representa la temperatura crítica de mojado. En este caso $T_{w}\left(h_{1}\right) \simeq 1,30(1)$.

dirección $y$, que son del orden de $L / 2$, para la elección de geometría propuesta en la ecuación (5.28).

La Figura (5.5) muestra un análisis de escala de las alturas y las ubicaciones de los picos de la susceptibilidad $k_{B} T \chi$, ecuaciones (5.23) y (5.24), respectivamente. Además, la figura (5.6) presenta el colapso de las curvas mostradas en la figura (5.3) cuando $m$, $m^{2}$ y $U$ se trazan en función de $\left(T-T_{w}\right) M^{1 / 2}$ como prueba de la ecuación (5.16), donde se utiliza la ley de potencia asintótica para $\chi_{\|}, \chi_{\|}=\hat{\chi}_{\|} t^{-v_{\|}}=\hat{\chi}_{\|} t^{-v_{2}}$. Es evidente que una buena cantidad de datos colapsan, aunque existen desviaciones debidas tanto a 

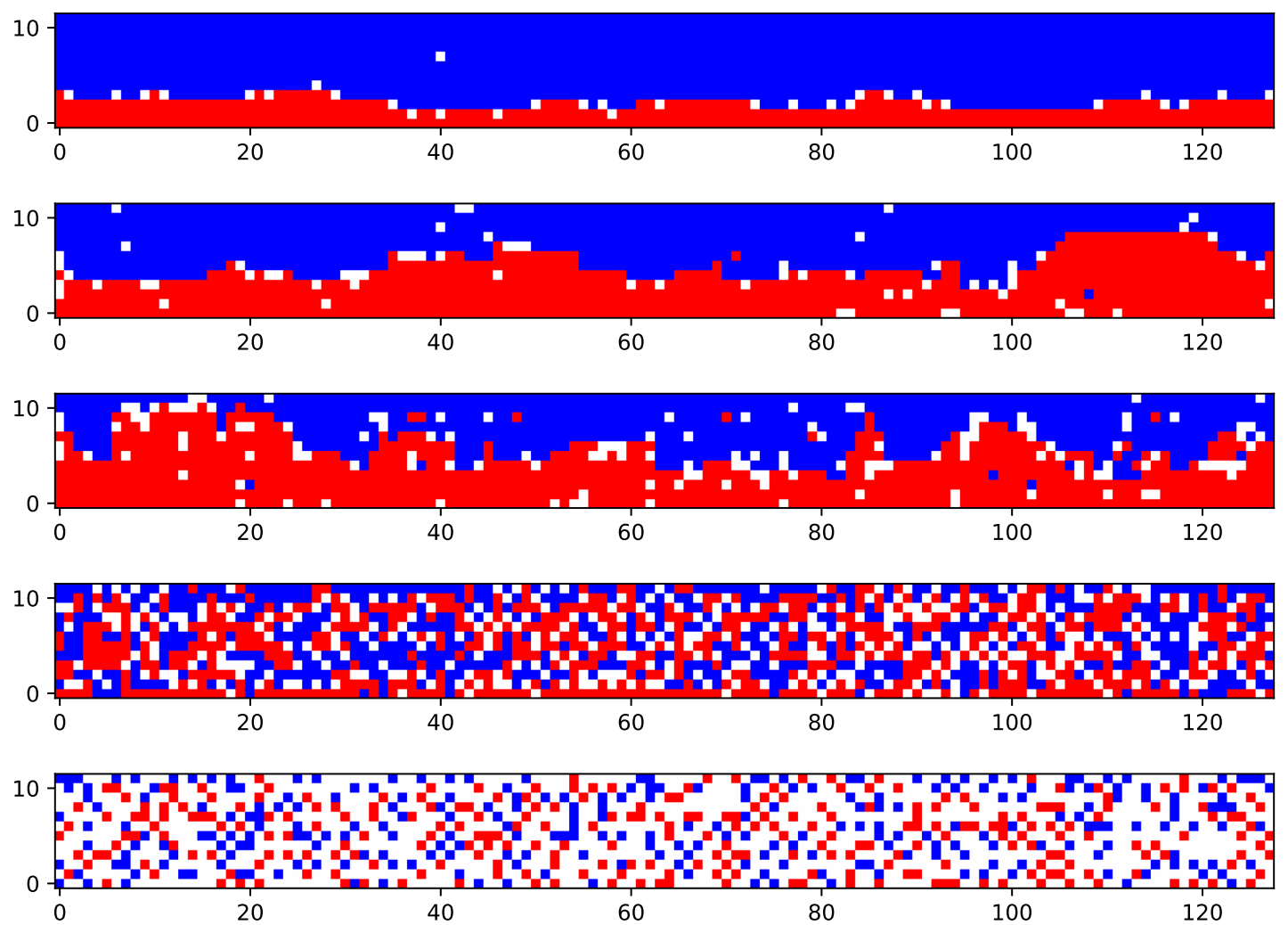

FIGURA 5.4. Snapshots de las configuraciones de espines que presenta el modelo BEG para distintas temperaturas. Los parámetros elegidos son $\alpha=1, D=$ $3, h_{1}=0,7$ y $(L, M)=(12,128)$. (a) muestra al sistema en $T=1,13$, es decir, por debajo de $T_{w}=1,30$. Se puede ver la interfase unida a la pared inferior con muy pocas vacancias(puntos blancos) acomodadas sobre ella. (b) corresponde a $T=1,28$, es decir, levemente por debajo de de $T_{w}$ pero lo suficientemente grande como para que la interfase se haya separado de la pared. (c, d, e) representan temperaturas por arriba de $T_{w}$, donde la interfase está deslocalizada y realiza excursiones entre las superficies de la muestra. El sistema se va poblando con vacancias para terminar en la fase paramagnética, completamente desordenado y lleno de vacancias.

errores estadísticos como a efectos sistemáticos, cuando $M$ y/o $\chi$ no son lo suficientemente grandes o cuando se incluyen datos demasiado lejos de $T_{w}\left(H_{1}\right)$.

Las figuras $(5.3,5.4,5.5,5.6)$ son solo ejemplos, pero son representativas del patrón general de comportamiento. Por lo tanto, se puede concluir que mediante el uso del análisis de escala de tamaño finito como el presentado aquí, se pueden localizar transi- 

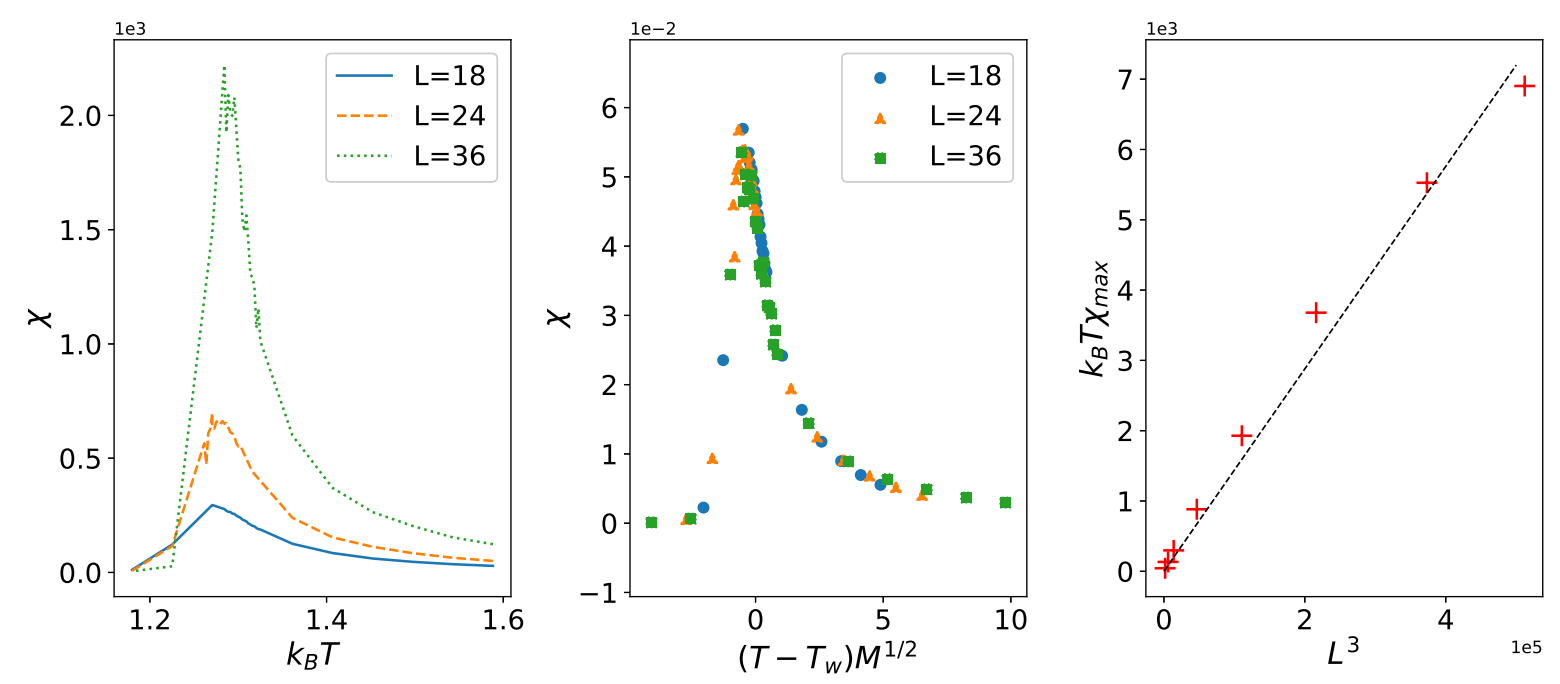

FiguRA 5.5. Gráfico de la susceptibilidad $\chi v s$ la temperatura $k_{B} T$ (izquierda) para los parámetros $\alpha=1, D=3, h_{1}=0,7$. Colapso de los datos de $\chi v s$ $\left|T-T_{w}\right| M^{1 / 2}$ (centro) el cual corrobora la ecuación (5.16). Más detalles en el texto. Por último, extrapolación de los picos de $\chi$ para demostrar la ley de potencia $k_{B} T \chi_{\max } \propto L^{3}$ ó $M^{3 / 2}$, ecuación (5.23).

ciones de mojado para el modelo de Blume-Emery-Griffiths en una red cuadrada, con una precisión razonable.

Luego, sobre la base de los resultados obtenidos, las figuras (5.7) y (5.8) muestran los diagramas de fase de las transiciones de mojado, es decir, gráficos de los puntos críticos en el plano $h_{s} v s T_{w} / T_{c b}$, y $D$ vs $T$ respectivamente. En la figura (5.7) se puede ver cómo, a medida que decrece el campo superficial, la temperatura crítica de mojado tiende a la temperatura crítica de Bulk. Por otro lado, para transiciones de mojado de segundo orden, la función inversa $h_{s}(T)$ de $T_{w}\left(h_{s}\right)$ se comporta como

$$
h_{s}(T) \propto\left(T_{c b}-T_{w}\right)^{\Delta_{1}}
$$

donde $\Delta_{1}$ es el exponente crítico que controla el comportamiento de escala con el campo superficial $h_{s}$ cerca de la criticalidad del bulk. La solución exacta de Abraham [8] da $\Delta_{1}=1 / 2$. Entonces, la figura (5.7) muestra $h_{w} v s\left(T_{c b}-T_{w}\right)^{1 / 2}$, como lo sugiere la ecuación (5.29). 


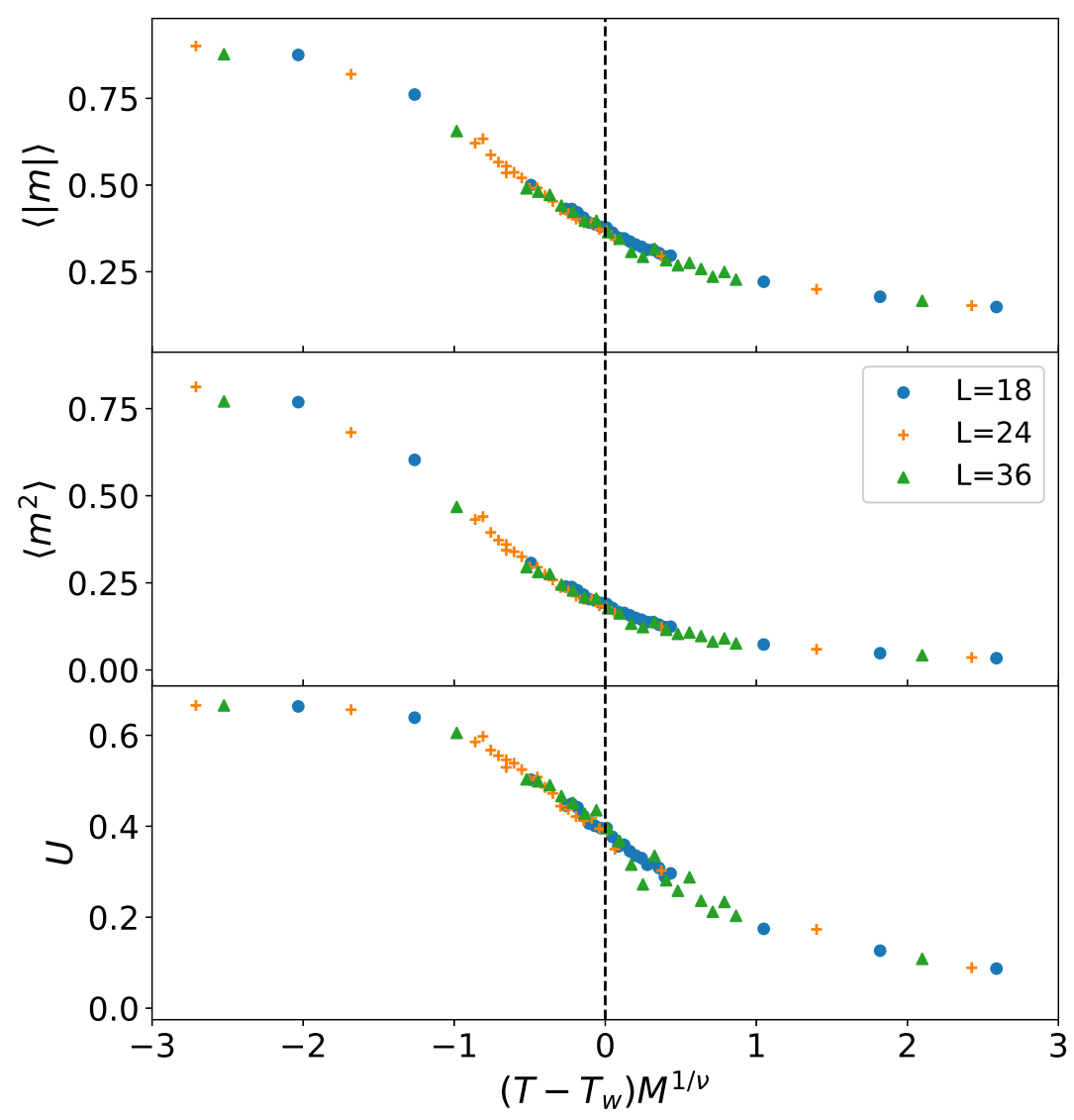

Figura 5.6. Gráficos de escala para $\langle|m|\rangle$ (arriba), $\left\langle m^{2}\right\rangle$ (centro) y $U$ (abajo), vs $\left(T-T_{w}\right) M^{1 / v}$, donde $v=2$. Para los parámetros $H_{w}=0,7, \alpha=1, D=3, \mathrm{y}$ distintos valores de $L$, como se muestra en la figura.

Estos diagramas se pueden incluir en el diagrama de fases del modelo BEG obtenido en el capítulo anterior, tal y como muestra la figura(5.8) para una mejor visualización de los resultados. 

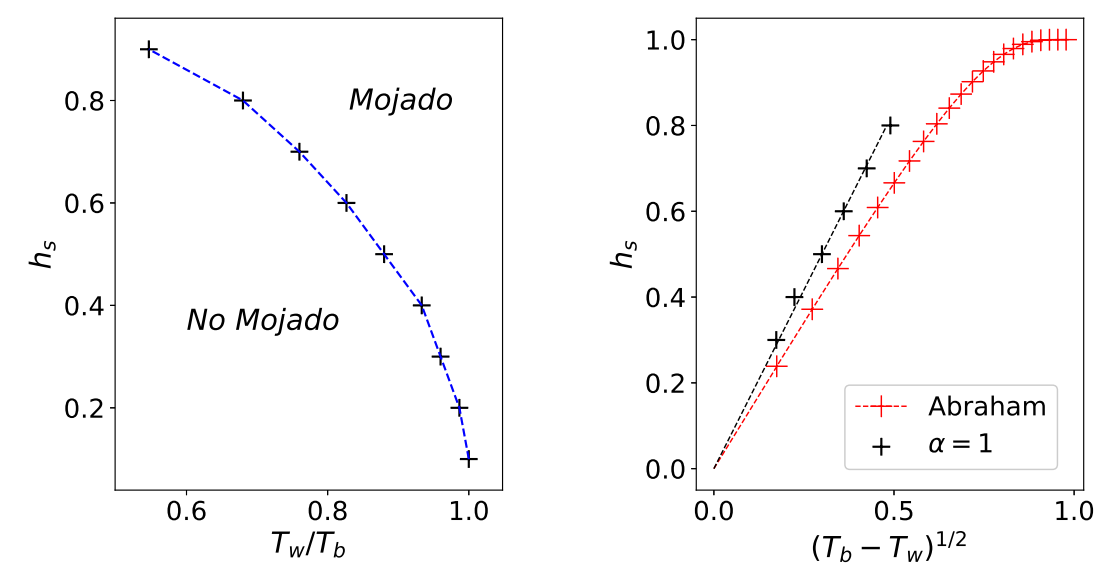

Figura 5.7. Izquierda: Gráfico de las transiciones de mojado en el plano del campo superficial $h_{s} v s T_{w} / T_{c b}$, con $T_{c b}=1,748(2)$. Los parámetros utilizados fueron $\alpha=1, D=3$. Derecha: Gráficos de los campos críticos de mojado $h_{s} v s\left(T_{c b}-T_{w}\right)^{\Delta_{1}}$, con $\Delta_{1}=1 / 2$, obtenidos para la solución exacta de Abraham $(\alpha=0)$ y los datos numéricos mostrados en el panel de la izquierda.

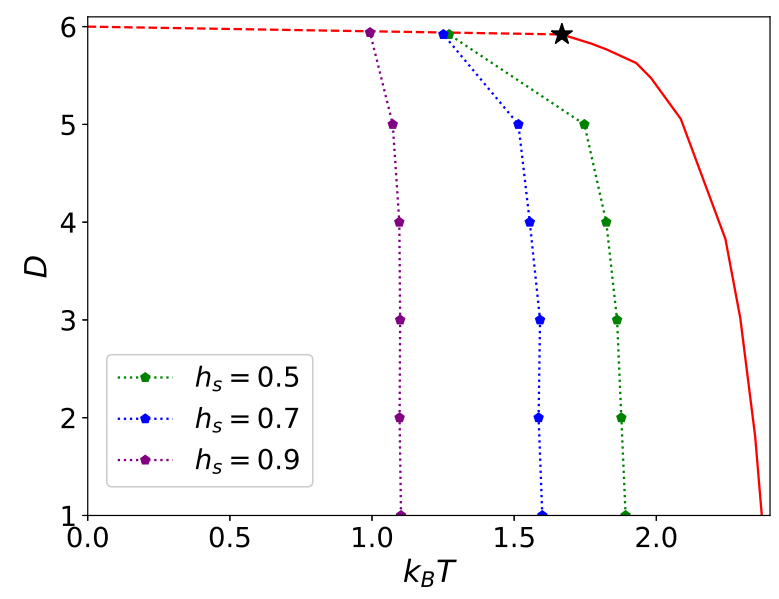

FiguRA 5.8. Gráfico de las transiciones de mojado, esta vez en el plano $D$ vs $k_{B} T$, para $\alpha=2$ y distintos valores de campo $h_{s}$ como se muestra en la figura. La línea roja representa el diagrama de fases de la figura (4.2) para $\alpha=2$, en ausencia de campos superficiales. La estrella negra representa el punto tricrítico. 


\subsection{Adsorción Interfacial}

Un aspecto interesante a analizar, es el enriquecimiento de vacancias en la interfase entre dominios con magnetización positiva y negativa, que resulta evidente al observar los snapshots de la figura (5.4). La figura (5.9) muestra gráficos de los perfiles de la magnetización (izquierda) y de las vacancias (derecha) para distintas temperaturas: $T<T_{w}$ en los paneles superiores y $T>T_{w}$ en los paneles inferiores. A medida que se aproxima a la $T_{w}$ la interfase se separa de la pared, y se ubica en el medio de la red, donde se observa un claro enriquecimiento de las vacancias. Para bajas temperaturas, la magnetización tiende a $\langle m\rangle=-1$, es decir, la concentración de vacancias es muy pequeña y predomina el dominio negativo. Mientras que, a medida que aumenta la temperatura, la concentración de vacancias aumenta y el dominio positivo empieza a ocupar parte de la red. Cuando $T \geq T_{w}$ la interfase entre el dominio positivo y el negativo se mueve libremente en el medio de la red y las vacancias se acomodan sobre ella, mostrando un claro enriquecimiento de vacancias en el panel inferior derecho. Cuando $T$ supera la temperatura crítica de Bulk, el sistema se empieza a poblar de vacancias, las cuales ocuparán casi toda la red, tal y como se ve en la figura (5.4(e)).

Una cantidad importante a medir es la adsorción interfacial $W_{0}$. Esta mide el excedente de vacancias debido a la interfase de la siguiente manera:

$$
W_{0}=L\left(f_{0}-F_{0}\right)
$$

donde $f_{0}$ es la densidad de vacancias cuando el sistema se encuentra bajo los efectos de campos superficiales inhomogéneos $\left(h_{1}=-h_{L}\right)$, mientras que $F_{0}$ representa lo mismo pero para campos homogéneos $\left(h_{1}=h_{L}\right)$.

Para determinar $F_{0}$ es necesario realizar nuevas simulaciones MC donde el sistema se encuentra bajo dicha condición.

La figura (5.10) muestra que, para $T_{w}, W_{0}$ presenta un punto de inflexión, por 

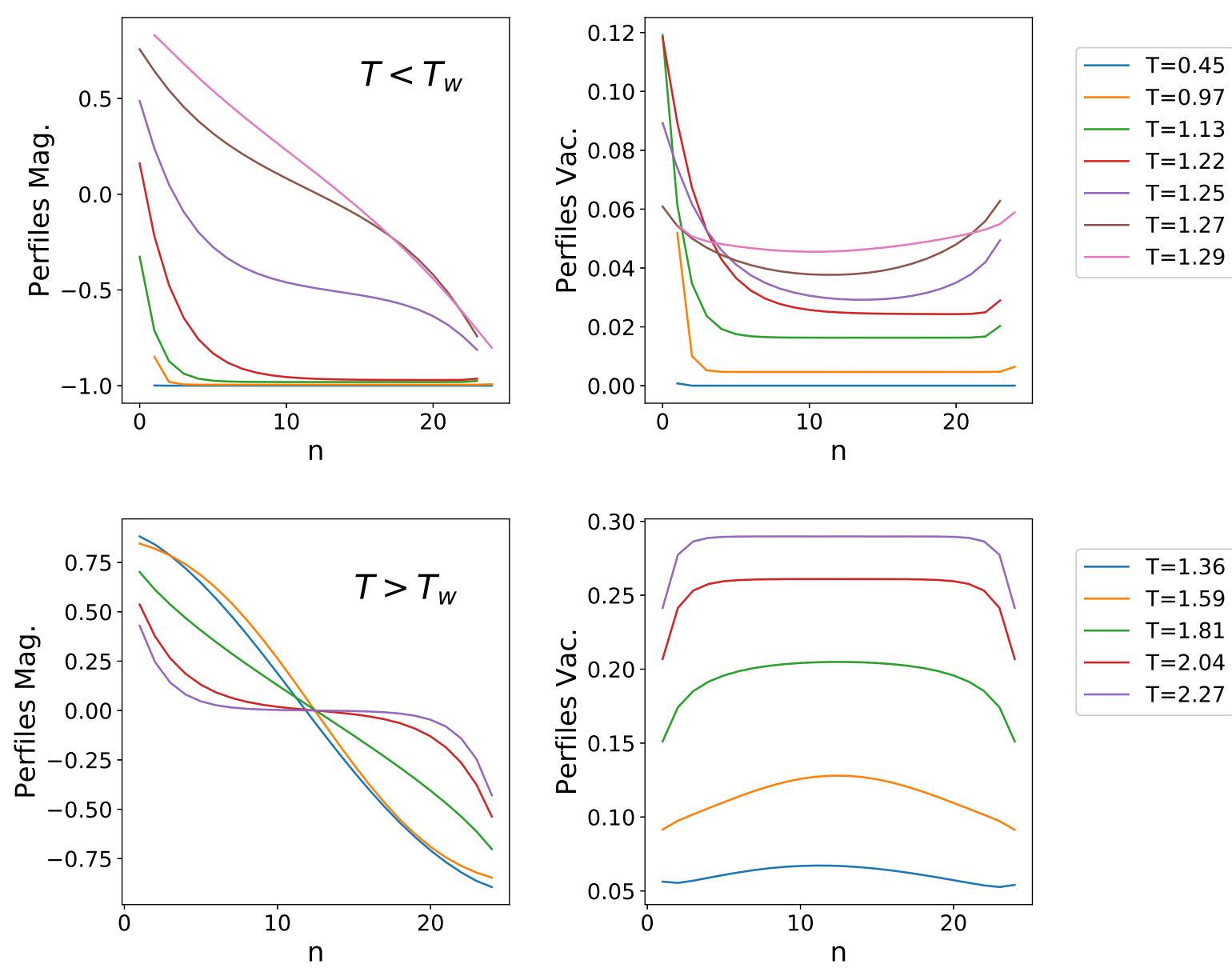

FIgURA 5.9. Perfiles de magnetización (izquierda) y de vacancias (derecha). Se representan en función de la distancia desde la pared $(n)$ sobre la que actúa el campo superficial positivo. Los datos corresponden a una red con longitudes lineales $(L, M)=(24,288)$ con parámetros $\alpha=1, D=3, h_{s}=0,7$ y distintos valores de temperatura, por encima (paneles inferiores) y por debajo (paneles superiores) de $T_{w}=1,30(1)$, como se muestra en la figura.

lo que si se realiza la derivada con respecto a la temperatura de dicha cantidad, la transición de mojado se verá caracterizada por un pico, mientras que para temperaturas lo suficientemente altas, aparecerá un pico negativo, el cual representa la transición de Bulk.

En la figura (5.11) se muestra dicho comportamiento para $\alpha=1, D=3$. En el panel izquierdo, se puede ver cómo los picos correspondientes a cada $h_{s}$ se ubican según lo 

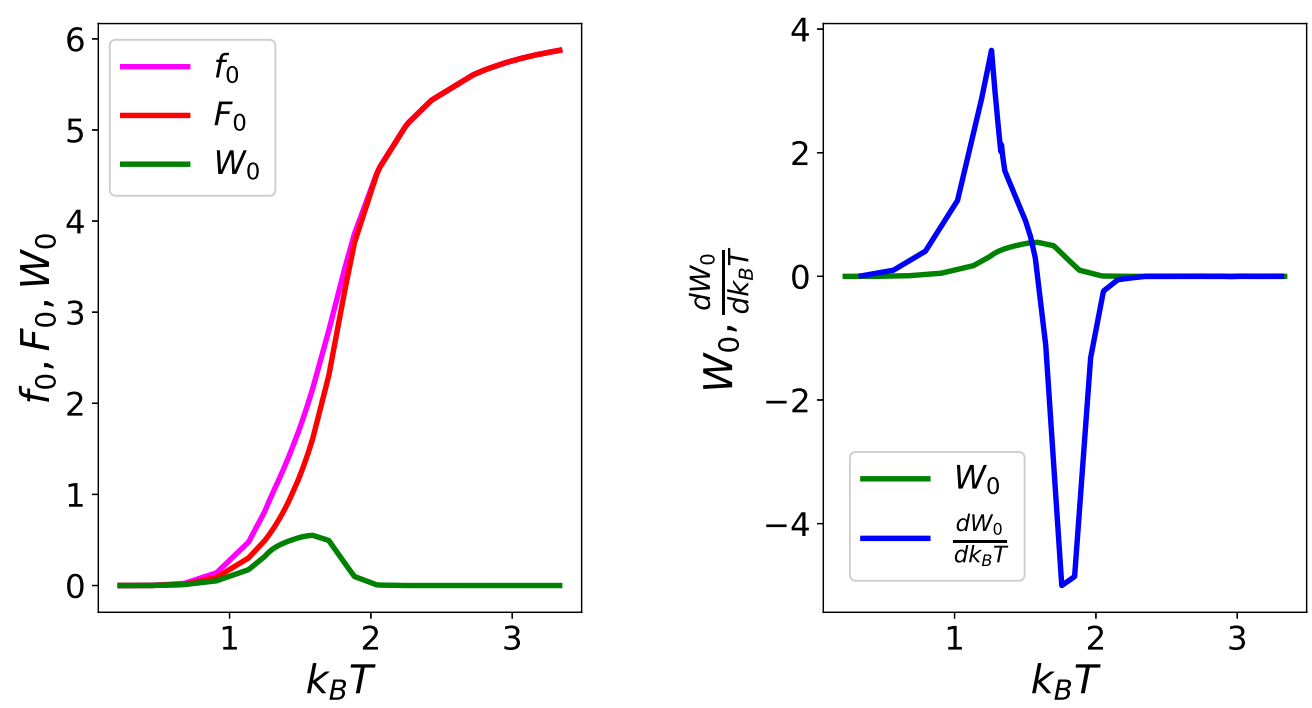

FiguRA 5.10. Gráficos de la adsorción interfacial para un sistema con $\alpha=$ $1, D=3$ y $h_{s}=0,7$. En el panel izquierdo se muestran las densidades de vacancias para un sistema con interfase $\left(f_{0}\right)$ y sin interfase $\left(F_{0}\right)$, y la diferencia de estas dos $\left(W_{0}\right)$, todas en función de la temperatura. Se observa claramente el enriquecimiento de vacancias debido a la interfase cerca de la temperatura crítica de wetting $T_{w}=1,30(1)$. En el panel derecho se muestra $W_{0}$ y su derivada con respecto a la temperatura. Se observa que el máximo corresponde a la transición de mojado, mientras que el pico negativo corresponde a la transición de Bulk para $T_{b}=1,748(2)$.

mostrado en la figura (5.7), mientras que el pico correspondiente a $T_{c b}$ es el mismo independientemente del campo. En el panel derecho se muestra la transición de mojado en función del tamaño de la red. Estos estudios coinciden con los presentados en la sección anterior, por lo que se puede concluir que el estudio de la adsorción interfacial permite obtener las transiciones de mojado de manera precisa.

Por otro lado, la figura 5.12 muestra la adsorción interfacial en función de la interacción bicuadrática $\alpha$. Si se mantiene $D=c t e$, a medida que $\alpha$ aumenta, tal y como se muestra en el inset, se observa que no solo decrece la adsorción interfacial, sino que aumenta la temperatura crítica de wetting. Esto ocurre ya que la diferencia de vacancias causada por la interfase, aumenta a medida que el sistema se aproxima a la fase paramagnética. Para el caso de $\alpha=1$ el sistema se encuentra próximo a dicha fase, por lo que 

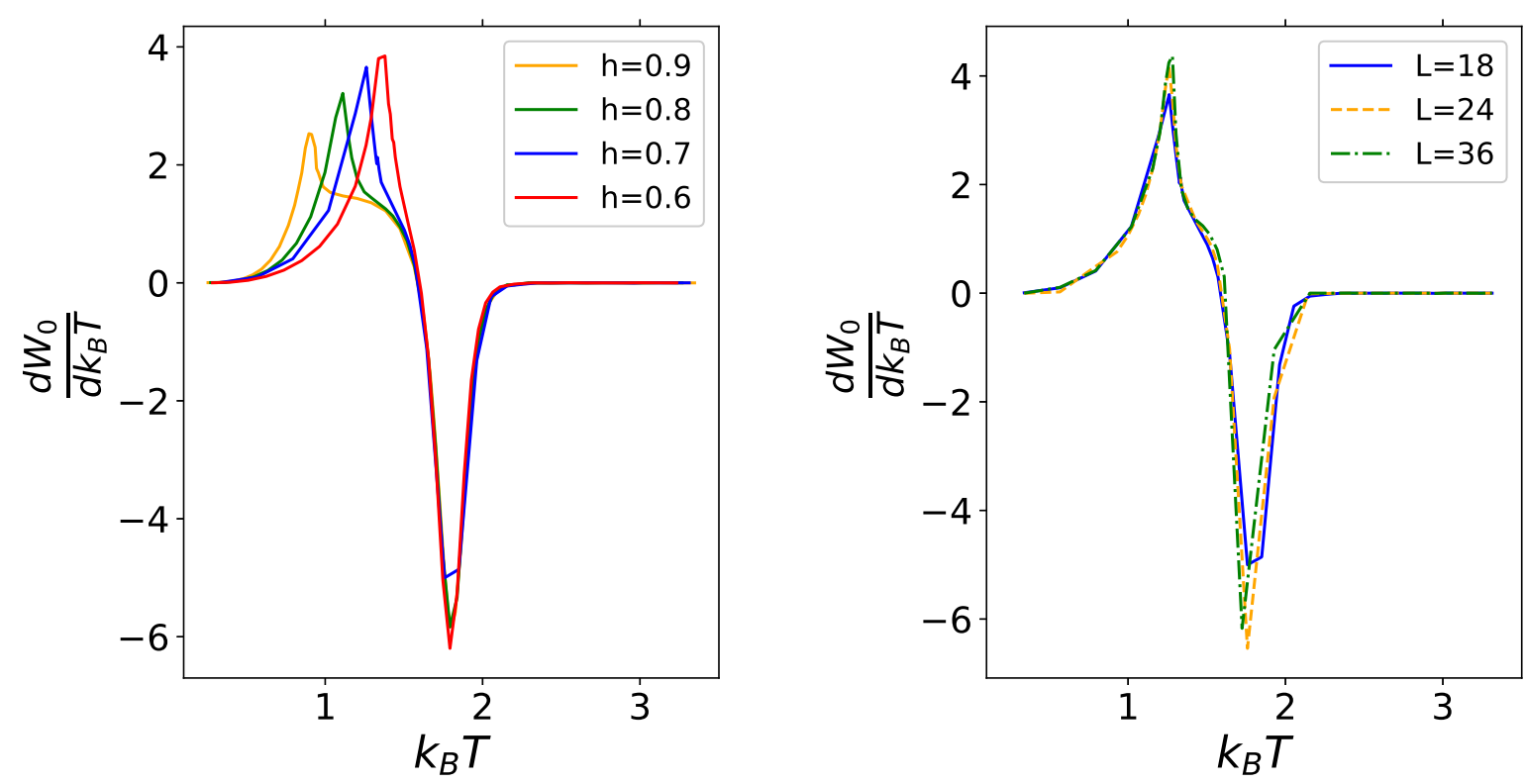

FiguRA 5.11. Gráficos de la derivada de la adsorción interfacial de vacancias, $W_{0}$, con respecto a la temperatura $v s$ la temperatura $k_{B} T$ para los parámetros $\alpha=1, D=3$ y: distintos valores del campo $h_{s}$ (izquierda) y distintos tamaños de red (derecha). Para dichos parámetros, $T_{w}\left(h_{s}=0,7\right)=1,30(1)$ (pico máximo en el panel derecho) y $T_{c b}=1,748(2)$ (pico mínimo en ambos paneles)

el pico de la adsorción interfacial es mayor que el de $\alpha=4$, que se encuentra muy lejos es esta. Es importante destacar que por cuestiones de escala, tanto la curva de $\alpha=3$ como la de $\alpha=4$ fueron multiplicadas por 10 y 100 respectivamente, para facilitar la comparación de éstas con la de $\alpha=1$. Por otro lado, a medida que aumenta $\alpha$, aumenta la $T_{c w}$ ya que la atracción entre los espines hace que a la interfase le cueste más desprenderse de la pared. 


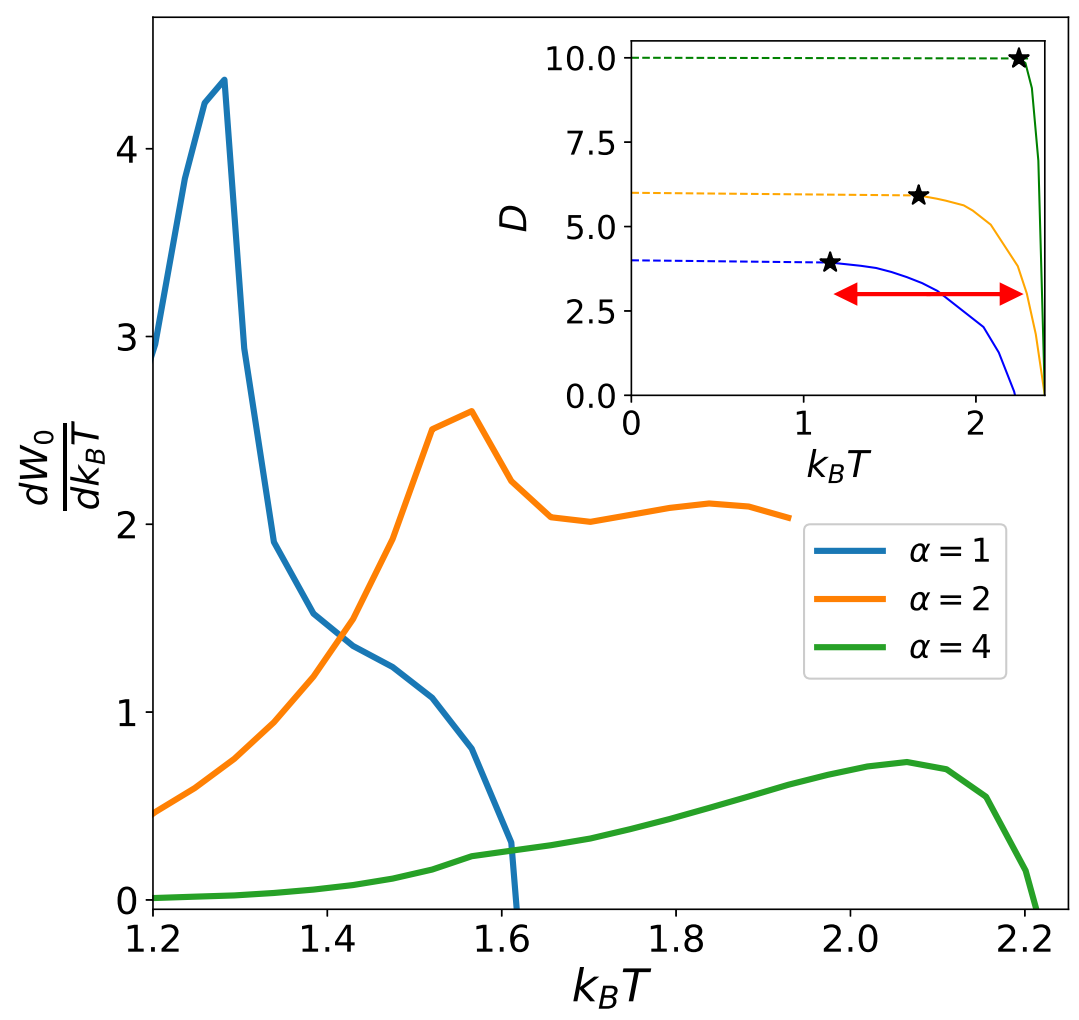

FigURA 5.12. Gráficos de la derivada con respecto a la temperatura de la adsorción interfacial de vacancias $W_{0} v s$ la temperatura $k_{B} T$ para los parámetros $D=3, h_{s}=0,7$ y distintos valores de $\alpha$. En el inset se puede observar la región del diagrama de fases que se está estudiando. Se puede observar que la región de estudio se aleja de la fase paramagnética a medida que aumenta $\alpha$. Por otro lado, A medida que aumenta $\alpha$, la adsorción interfacial disminuye, pero aumenta la $T_{w}$.

\subsection{Integración Termodinámica}

Otra forma de obtener las transiciones de mojado, en particular aquellas de primer orden, es mediante la Integración Termodinámica. Este método permite determinar dónde se localizan los puntos de coexistencia en el diagrama de fases o, en otras palabras, hallar para cada temperatura los valores de campo donde ocurren las transiciones de fase. El mismo ha sido utilizado previamente con éxito para localizar transiciones de mojado en $d=3[8]$ y $d=2[27,84]$ dimensiones. Para una descripción y discusión más detallada 
sobre éste método, referirse a [86, 87].

Con el fin de localizar las transiciones de mojado, se considera el hecho de que éstas dependen de la diferencia del exceso de energía libre superficial $\left(\Delta f_{1 L}=f_{s}^{+}-f_{s}^{-}\right)$ entre dominios semi infinitos con magnetizaciones positivas (+) y negativas (-). Los signos de las magnetizaciones son causados por los campos superficiales y por la tensión interfacial entre las fases coexistentes $f_{i n t}(T)$. Ésta, según el criterio de Young [83], cumple $f_{s}^{+}-f_{s}^{-}=f_{\text {int }}$. Para el modelo de Ising, la energía libre interfacial se conoce de manera exacta gracias a la solución de Onsager [88],

$$
\sigma=2 J+k_{B} T \log \left[\tanh \left(\frac{J}{k_{B} T}\right)\right] .
$$

Pero en este trabajo se consideran sistemas con impurezas no magnéticas, es decir, la solución de Onsager no se aplica. Por tal motivo es necesario recurrir a la integración termodinámica. En primer lugar se utilizan las relaciones

$$
\begin{aligned}
& m_{1}=-\left(\frac{\partial f_{s}\left(T, h_{1}\right)}{\partial h_{1}}\right)_{T} \\
& m_{L}=-\left(\frac{\partial f_{s}\left(T, h_{L}\right)}{\partial h_{L}}\right)_{T},
\end{aligned}
$$

las cuales conectan la magnetización de la superficie en las paredes 1 y $L$, con la energía libre superficial y el campo superficial correspondiente. De esta manera, la diferencia del exceso de energía libre superficial $\left(\Delta f_{1 L}\right)$, se puede obtener de la siguiente manera

$$
\begin{aligned}
\Delta f_{1 L} & =f_{s}\left(T, h_{1}\right)-f_{s}\left(T, h_{L}\right), \\
& =\int_{0}^{h_{1}}\left(m_{L}-m_{1}\right) d h_{1}^{\prime}
\end{aligned}
$$

Para evaluar esta integral, se tomaron $h_{L}=-h_{1}=0$ con un sistema de tamaño $L=36$ y $M=1152$, cuya configuración inicial consistía en todos los espines apuntando hacia arriba. Además, se tomaron promedios de más de $10 \times 10^{6} \mathrm{MCS}$, después de descartar los primeros $2 \times 10^{6}$ MCS. Como prueba de la precisión de este procedimiento, primero se estudió el caso $D=-\infty$, en el cual se excluyen las vacancias y se obtiene el problema 
estándar de wetting en el modelo de Ising $d=2$, para el cual se conoce la solución exacta gracias a Abraham [8].

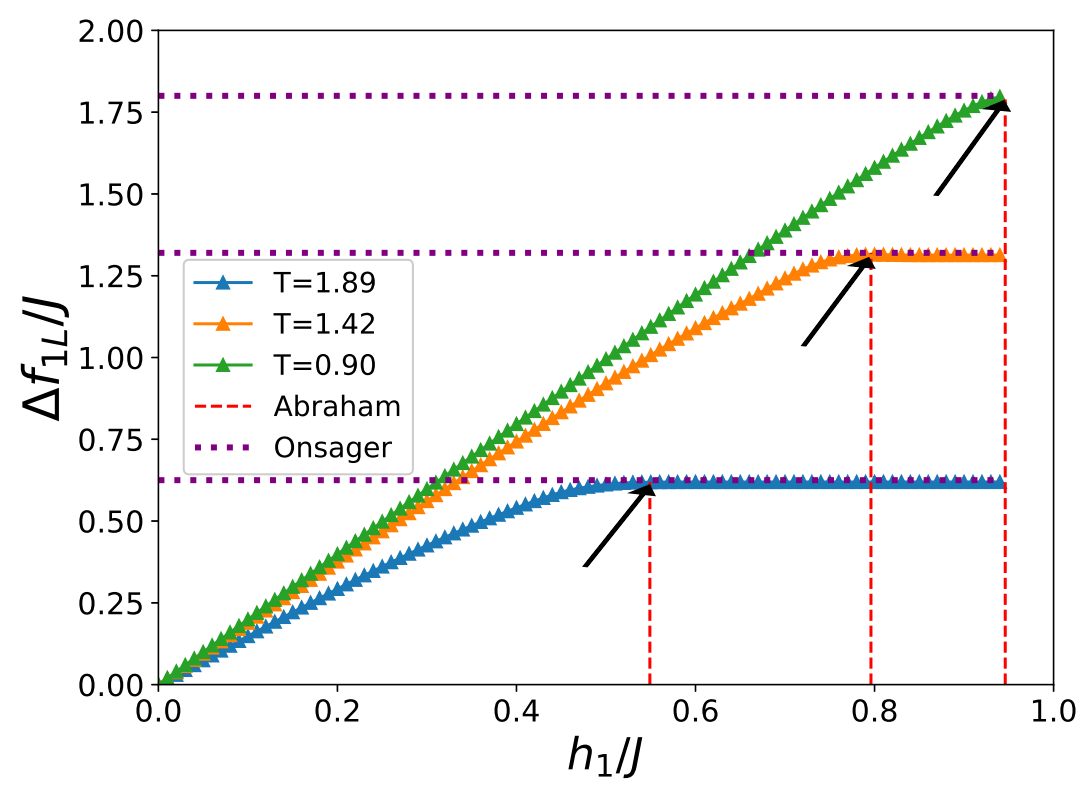

FigurA 5.13. Gráficos de $\Delta f_{1 L} / J$ en función del campo superficial $h_{1} / J$, obtenidos para distintas temperaturas, con $\alpha=0$ y $D=-\infty$, es decir, el modelo de Ising. Las líneas horizontales corresponden a la energía libre interfacial, la cual se obtiene a partir de la solución exacta de Onsager [88], mientras que las líneas verticales corresponden a los campos críticos para los cuales ocurren las transiciones de mojado, y se obtienen de la solución exacta de Abraham [8]. Los puntos de intersección, están indicados con flechas.

La figura (5.13) muestra los resultados para $\Delta f_{1 L}$ en función de $h_{1}$ obtenido para $D=-\infty$. Se encontró que la ecuación (5.34) se puede discretizar en pasos de $h_{1}=0,025$ para que el error de integración numérica sea lo suficientemente pequeño (tenga en cuenta que los campos también se indican en unidades de $J$ ). Dado que para el caso del modelo Ising, la energía libre interfacial se conoce exactamente según Onsager (5.31), en la figura (5.13) se incluyeron los valores de $\sigma$ correspondientes a la temperatura para la que se realizó la integración de la ecuación (5.34) (líneas horizontales). Además, se incluyeron los resultados exactos de Abraham para los campos críticos de las transiciones de mojado (líneas verticales), que interceptan las curvas de integración justo cuando 
comienzan a saturarse y coinciden (dentro del error estadístico) con las líneas que definen la energía libre interfacial. Por lo tanto, la figura (5.13) muestra que mediante el uso de la integración termodinámica y aprovechando la existencia de resultados exactos, la ubicación de las transiciones de mojado es compatible con los resultados exactos.

Desafortunadamente, para el caso de $D>-\infty$, no existe una solución exacta de $\sigma$, por lo que la energía libre interfacial también debe encontrarse mediante la integración termodinámica.

Para ello, se utilizan dos estados de referencia del sistema: uno con todos los espines apuntando para arriba (+1), y otro en el que los espines en contacto con la pared, cuyo campo superficial es $+h_{1}\left(-h_{L}\right)$, apunten para arriba (abajo). De esta manera, usando la relación entre la energía libre y la energía interna por espín [86, 87]

$$
u=\left(\frac{\partial(\beta f)}{\partial \beta}\right)_{\alpha, D, h_{1}, h_{L}},
$$

donde $\beta=1 / T$, se puede realizar la siguiente integración

$$
\beta f(\beta)=\beta_{0} f\left(\beta_{0}\right)+\int_{\beta_{0}}^{\beta} u\left(\beta^{\prime}\right) d \beta^{\prime} .
$$

Aquí $\beta_{0}$ representa un estado de referencia y $\beta$ al estado en la temperatura de trabajo, es decir, la temperatura en la cual se pretende encontrar la transición. La opción más tentadora es tomar $\beta_{0}=0$, pero, dado que se analizarán temperaturas menores a la temperatura crítica del sistema, ésto no resulta conveniente. Una alternativa es considerar como estado de referencia a $T=0$, donde la entropía es nula y se puede considerar la energía libre igual a la energía interna en virtud de la relación

$$
U=F-T S
$$

En la implementación numérica bastará con tomar una temperatura no nula pero muy pequeña para considerar irrelevantes las contribuciones entrópicas. Por ejemplo, $T=0,05$, es decir $\beta_{0}=20$, es lo suficientemente pequeña para omitir dichas contribuciones. 
Este procedimiento se ve simplificado cuando se utiliza el algoritmo de Wang-Landau, el cual permite calcular de forma directa la energía libre del sistema. Para ello se calcula la función de partición de los dos estados de referencia: uno con todos los espines apuntando para arriba y el otro con una mitad apuntando para arriba y la otra para abajo. Es importante destacar que para obtener la interfase en éste último caso, se utilizaron condiciones antiperiódicas de contorno. Luego, se calcula la energía libre para ambos casos y se restan de la siguiente manera

$$
f_{\text {interfacial }}=f^{ \pm}-f^{+}
$$

donde $f^{ \pm}$es la energía libre del sistema con interfase y $f^{+}$es la energía libre del sistema sin interfase. Ya que el exceso de energía en $f^{ \pm}$es causado por la interfase, $f_{\text {interfacial }}$ es la energía libre interfacial. De esta manera es posible calcular dicha energía para cualquier valor de $\alpha$ y $D$.
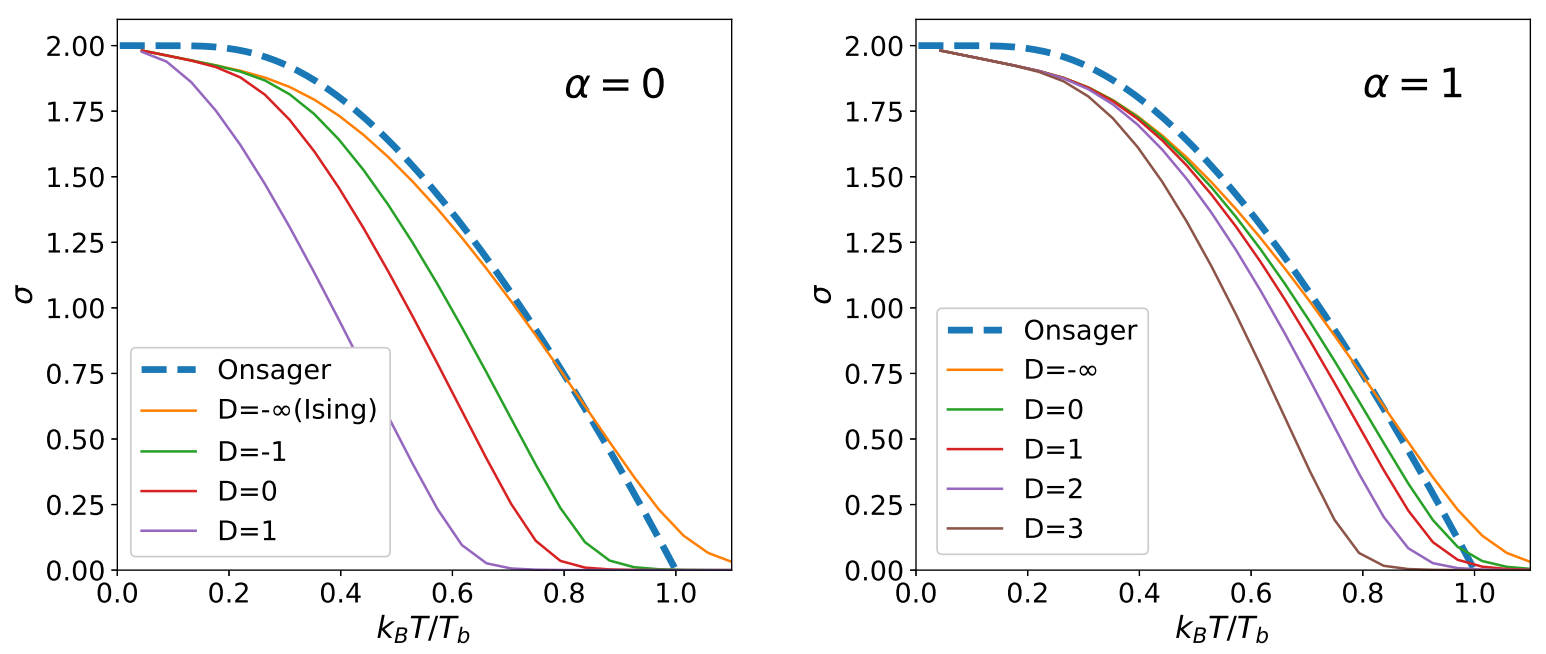

FigurA 5.14. Gráficos de la energía libre interfacial $\sigma$ en función de $k_{B} T / T_{b}$, para $\alpha=0$ (izquierda) y $\alpha=1$ (derecha), con distintos valores de $D$. Los resultados obtenidos para el modelo de Ising $(D=-\infty$ y $\alpha=0)$ presentan un pequeño corrimiento con respecto a la solución exacta de Onsager (línea punteada), debido al tamaño de la red $(L=16)$.

La figura (5.14) muestra gráficos de $\sigma$ en función de $k_{B} T / T_{b}$ obtenidos para diferentes 
valores de $D$ y de $\alpha$. Para el caso de prueba $D=-\infty$, las barras de error de la integración numérica son más pequeñas que el tamaño de la línea. Por otro lado, para valores finitos de $D$, la energía libre interfacial disminuye (a temperatura constante) en comparación con el caso de Ising debido a la presencia de impurezas no magnéticas en la interfaz, tal y como se esperaba.

Por último, la figura (5.15) muestra los gráficos de los resultados de la integración de $\Delta f_{1 L}$ en función de $h_{1}$, obtenidos para $D=3, \alpha=1$ y diferentes valores de temperatura. $\mathrm{Al}$ igual que en la figura (5.13), se incluyeron los valores de $\sigma$ obtenidos mediante integración termodinámica, y los valores de los campos críticos, obtenidos a partir de los gráficos de la figura (5.7). Los resultados coinciden con aquellos mostrados en las figuras $(5.5,5.6$ y 5.11$)$ para $h=0,7$.

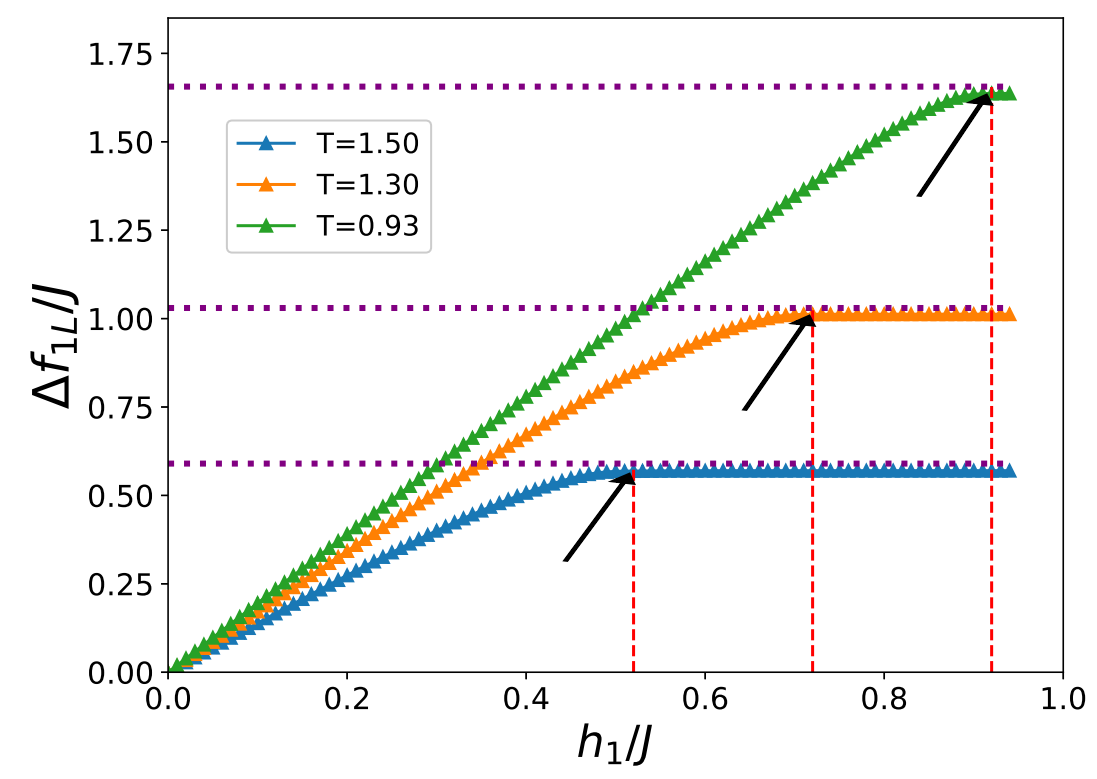

Figura 5.15. Gráficos de $\Delta f_{1 L} / J$ en función del campo superficial $h_{1} / J$ obtenidos para distintas temperaturas, con $\alpha=1$ y $D=3$. Las líneas horizontales corresponden a la energía libre interfacial obtenida mediante integración termodinámica (mirar figura (5.14). Los puntos de intersección para las transiciones de mojado de segundo orden, están señalados con flechas. Es importante destacar que estos puntos coinciden con aquellos mostrados en la sección 5.2.1. 
Este método fue de gran utilidad para confirmar las transiciones de segundo orden. Por otro lado, es muy útil al momento de trabajar con transiciones de primer orden, en las cuales no es posible realizar escaleo de tamaño finito. Sin embargo, no se pudo hacer uso del mismo ya que no se encontraron transiciones de ese tipo en el modelo de BEG bajo confinamiento. 


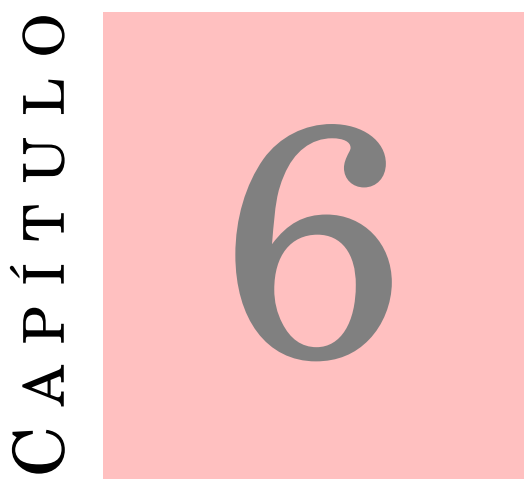

\section{ESTUDIO DEL CONFINAMIENTO EN EL}

\section{MODELO RBIM}

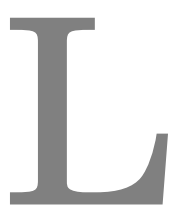

Los estudios del confinamiento que se realizaron hasta ahora consideraban desorden dinámico, es decir, las vacancias no estaban fijas o, en otras palabras, éstas podían crearse y/o destruirse en cualquier sitio de la red. Por otro lado, como se vio en los capítulos 4 y 5, los exponentes críticos variaban, pero siempre cumpliendo las relaciones de hiper escala. ¿Qué pasa cuando se congela el desorden? y más aún ¿Qué pasa con las relaciones de hiper escala si las longitudes de correlación decaen de forma distinta a la que se venía estudiando? En este capítulo se propone un estudio alternativo de los efectos del confinamiento con desorden congelado en el cual se viola la relación de hiper escala. Para ello se propone una nueva teoría de escala de tamaño finito para transiciones de mojado, independiente de las relaciones de hiper escala. 


\subsection{El Modelo}

El modelo elegido para estudiar el confinamiento con desorden congelado (quenched disorder en inglés) es el modelo de Ising con impurezas inmóviles o congeladas colocadas al azar, las cuales se generan a partir de constantes de acoplamiento aleatorias. Si los efectos de las impurezas son lo suficientemente débiles, el sistema se mantendrá ordenando y se puede considerar una interfase que separa dos dominios, uno con magnetización positiva y otro con magnetización negativa. Sin embargo, estas impurezas tenderán a fijar dicha interfase en ciertas ubicaciones donde las constantes de acoplamiento sean más débiles que el promedio. Por lo tanto, la posición de equilibrio de la interfase, se determina mediante la competencia entre los espines, que intentan minimizar el área de la interfase, y las impurezas, que quieren que la interfase pase por los lugares donde el sistema tiene la energía local más baja, volviéndola rugosa. El Hamiltoniano de este modelo tiene la siguiente forma

$$
\mathscr{P}^{R B I M}=-\sum_{\langle i, j\rangle} J_{i j} s_{i} s_{j},
$$

donde las variables de espín, $s_{i}$, sólo toman valores 1 y -1, y las constantes de acoplamiento aleatorias se eligen a partir de una distribución bimodal

$$
\mathscr{P}\left(J_{i j}\right)=\frac{1}{2}\left[\delta\left(J_{i j}-J_{1}\right)+\delta\left(J_{i j}-J_{2}\right)\right] .
$$

La temperatura crítica de bulk de éste modelo se puede obtener de manera exacta [89], mediante la siguiente ecuación, donde $k_{B}=1$

$$
\sinh \left(\frac{2 J_{1}}{T_{c b}}\right) \sinh \left(\frac{2 J_{2}}{T_{c b}}\right)=1
$$

Siguiendo a Fytas y Theodorakis [90], se eligieron los casos $J_{1}=4 / 3, J_{2}=2 / 3$ (eligiendo la escala de temperatura tal que $J_{1}+J_{2}=2$ ) de modo que $T_{c b}=2,18802, T_{c b} / J_{1}=1,64143$.

Para estudiar transiciones de mojado, dicho sistema se confinó a una geometría $L \times M$ con condiciones de contorno libres en $i=1$ e $i=L$, donde actúan los campos superficiales 
$h_{1}=-h_{L}<0$ respectivamente, y condiciones periódicas de contorno en la dirección $x$, paralela a las paredes. Por lo tanto, el Hamiltoniano final tiene la forma

$$
\mathscr{H}=\mathscr{P}^{R B I M}+h_{1} \sum_{i \in 1} s_{i}-h_{L} \sum_{i \in n=L} s_{i}
$$

\subsection{Violación de la hiper escala}

El efecto que causan las impurezas en la interfase, tiene implicaciones muy importantes al momento de estudiar fenómenos causados por el confinamiento, como ser las transiciones de mojado.

Como se discutió en el capítulo 5, dichas transiciones se pueden considerar como una transición de localización-deslocalización de la interfase con respecto a la pared y, como se dijo previamente, la rugosidad causada por las impurezas fijas, afectará el comportamiento de la interfase. Se ha demostrado [91] que dichas impurezas provocan cambios en las longitudes de correlación $\xi_{\|}$y $\xi_{\perp}$ del sistema, y por lo tanto en sus exponentes críticos $v_{\|}$y $v_{\perp}$ respectivamente, de modo que

$$
v_{\|}=3, \quad v_{\perp}=2 .
$$

Estos resultados son compatibles con los presentados por Huse et.al. [92, 93] pero violan la relación de hiper escala detallada en los capítulos 3 y 5

$$
(d-1) v_{\|}=2-\alpha_{s}
$$

para la cual $\alpha_{s}=0$. Sin embargo, el escaleo termodinámico (ecuación 5.3) es válido, como así también el hecho de que $\eta_{\|}=0$. Por lo tanto, haciendo uso de las relaciones de escala analizadas en el capítulo anterior, se obtiene:

$$
\begin{gathered}
\gamma_{s}=v_{\|}\left(2-\eta_{\|}\right)=6, \\
\Delta_{s}=\left(2-\alpha_{s}+\gamma_{s}\right) / 2=4, \\
\beta_{s}=2-\alpha_{s}-\Delta_{s}=-2 .
\end{gathered}
$$


Los exponentes críticos anteriores permiten ubicar a éste modelo dentro de la clase de universalidad de Régimen de fluctuación fuerte [91, 94].

A continuación se presentará un desarrollo de la teoría de escala de tamaño finito para el wetting crítico, análogo al del capítulo 5 , pero independiente de la ley de hiper escala.

La suposición de la teoría de escala presentada en el capítulo anterior fue que las transiciones de wetting en una geometría $L \times M$, la cual mantenía la relación de aspecto $c=L^{v_{\|} / v_{\perp}} / M$ constante, con dos campos superficiales competitivos $h$, se podían describir en términos de la función de distribución $P_{L, M}(m)$ (5.15) de la magnetización total del sistema. Esta función de distribución es claramente bimodal cuando la interfase está unida a cualquiera de las paredes, pero debe mostrar un pico amplio centrado en $m=0$ en la fase mojada. Se había dicho que

$$
P_{L, M}(m)=\xi_{\|}^{\beta / v_{\|}} \tilde{P}\left(c, \frac{M}{\xi_{\|}}, m \xi_{\|}^{\beta / v_{\|}}\right)
$$

donde se asume que $M$ escalea con $\xi_{\|}$y la magnetización total $m$ escalea como $t^{\beta} \propto \xi_{\|}^{\beta / v_{\|}}$. Manteniendo $c$ constante, no se considera $\xi_{\perp}$, y el prefactor $\xi_{\|}^{\beta / v_{\|}}$ubicado frente a la función de escaleo $\tilde{P}$, asegura la condición de normalización.

De la ecuación (6.8) se puede ver que

$$
\left[\left\langle m^{k}\right\rangle\right]_{a v}=\xi_{\|}^{-k \beta / v_{\|}} \tilde{m}_{k}\left(c, \frac{M}{\xi_{\|}}\right)
$$

donde $k=1,2, \cdots \mathrm{y}[\ldots]_{a v}$ es el promedio sobre el desorden congelado que presenta el sistema. De esta manera, la susceptibilidad se puede escribir como

$$
\begin{aligned}
k_{B} T \chi^{\prime}= & L M\left(\left[\left\langle m^{2}\right\rangle\right]_{a v}-[\langle|m|\rangle]_{a v}^{2}\right) \\
& =L M \xi_{\|}^{-2 \beta / v_{\|}} \tilde{\chi}\left(c, \frac{M}{\xi_{\|}}\right),
\end{aligned}
$$

donde $\tilde{m}_{k}$, la cual está definida de forma implícita en la ecuación (6.9) análogamente a las ecuaciones (5.16) y (5.17), y $\tilde{\chi}=\tilde{m}_{2}-\left(\tilde{m}_{1}\right)^{2}$ son las funciones de escala. 
Usando $L=c^{v_{\perp} / v_{\|}} M^{v_{\perp} / v_{\|}}$la susceptibilidad se puede escribir como

$$
\tilde{\chi}\left(c, \frac{M}{\xi_{\|}}\right) \equiv c^{v_{\perp} / v_{\|}}\left(\frac{M}{\xi_{\|}}\right)^{2 \beta / v_{\|}} \tilde{\chi}\left(c, \frac{M}{\xi_{\|}}\right)
$$

tal que,

$$
k_{B} T \chi^{\prime}=M^{1+v_{\perp} / v_{\|}-2 \beta / v_{\|}} \tilde{\tilde{\chi}}\left(c, \frac{M}{\xi_{\|}}\right) .
$$

Por lo tanto, la predicción del escaleo de tamaño finito para la susceptibilidad en la transición wetting es

$$
\left.k_{B} T_{w} \chi^{\prime}\right|_{T_{w}} \propto M^{1+v_{\perp} / v_{\|}-2 \beta / v_{\|}} .
$$

Por otro lado, según las relaciones que se desprenden de las ecuaciones (5.10), la susceptibilidad superficial escalea como

$$
\chi_{s}=\xi_{\|}^{2} \tilde{\chi}_{s}\left(\frac{M}{\xi_{\|}}\right)
$$

por tal motivo, en la transición de wetting, la susceptibilidad se comportará como $\chi_{s} \mid T_{w} \propto$ $M^{2}$. De la misma manera que en el wetting con impurezas dinámicas, la parte crítica de $\chi^{\prime}$ se debe solamente a $\chi_{s}$, por lo tanto

$$
\begin{gathered}
\left.k_{B} T_{w} \chi^{\prime}\right|_{T_{w}}=\left.L^{-1} k_{B} T_{w} \chi_{s}\right|_{w} \\
=c^{-v_{\perp} / v_{\|}} M^{-v_{\perp} / v_{\|}} k_{B} T_{w} M^{2} \\
\propto M^{2-v_{\perp} / v_{\|}}=M^{4 / 3} .
\end{gathered}
$$

Los exponentes $2-v_{\perp} / v_{\|}$deberían ser iguales a aquellos obtenidos en la ecuación (6.12), $1+v_{\perp} / v_{\|}-2 \beta / v_{\|}$, de modo que

$$
2 \beta=2 v_{\perp}-v_{\|}=1, \quad \beta=\frac{1}{2}
$$

Esto permite reescribir la ecuación (6.15) como

$$
\left.k_{B} T_{w} \chi^{\prime}\right|_{T_{w}} \propto M^{\gamma / v_{\|}}=M^{4 / 3} .
$$


La hiper escala, (implícita en la ecuación (6.8)) implica que $\gamma=4$, lo cual resulta compatible con la relación de hiper escala para el bulk de sistemas anisotrópicos

$$
v_{\|}+v_{\perp}=5=\gamma+2 \beta
$$

Pero, si bien se satisface la relación de escaleo

$$
\gamma_{s}=\gamma+v_{\perp}=6
$$

la relación

$$
\beta_{s}=\beta-v_{\perp}
$$

sería compatible con la ecuación (6.7), $\beta_{s}=-2$, sólo si $\beta=0$. La misma conclusión se obtiene cuando se usan las relaciones de escala termodinámicas [62]

$$
\Delta_{s}=\gamma+\beta=4
$$

Por lo tanto, existe una contradicción entre el escaleo termodinámico que requiere

$$
\beta=0
$$

y el de hiper escala, el cual requiere $\beta=1 / 2$ [93]. El resultado (6.22) implica que

$$
[\langle|m|\rangle]_{a v}=\tilde{m_{1}}\left(c, \frac{M}{\xi_{\|}}\right)
$$

y

$$
\left[\left\langle m^{2}\right\rangle\right]_{a v}=\tilde{m_{2}}\left(c, \frac{M}{\xi_{\|}}\right) .
$$

Como consecuencia se espera que en la transición de wetting, donde $\xi_{\|} \rightarrow \infty$, estos momentos se vuelvan independientes de la dimensión lineal. De esta manera, al graficar $[\langle|m|\rangle]_{a v} \mathrm{y}\left[\left\langle m^{2}\right\rangle\right]_{a v}$ vs $t$ deberían observarse los puntos de transición independientes del tamaño del sistema, $\tilde{m}_{1}(c, 0)$ y $\tilde{m_{2}}(c, 0)$, para $t=0$.

Como se vio en el capítulo anterior, esta propiedad se cumple para las transiciones críticas de wetting, pero para el caso del $R B I M$, en el cual se viola la relación de hiper 
escala (6.6), ambos escaleos (termodinámico y de hiper escala) implican resultados contradictorios. En la siguiente sección se darán pruebas, basadas en simulaciones numéricas, sobre la violación de hiper escala.

\subsection{Resultados}

\subsubsection{Detalles sobre las simulaciones}

Teniendo en cuenta que es útil mantener la relación de aspecto generalizada $c=$ $L^{v_{\|} / v_{\perp}} / M=L^{3 / 2} / M$ constante, y que las longitudes lineales $L$ y $M$ de la red deben ser enteros, es fácil ver que estos requisitos se cumplen de manera trivial si $L$ y $M$ se eligen como segunda y tercera potencia de un entero. Por lo tanto, se han utilizado las opciones $(L, M)=(25,125) ;(36,216) ;(49,343)$ y $(64,512)$. Como al estudiar fenómenos críticos es necesario que $L \gg 1$, no se consideraron longitudes lineales más pequeñas. Se utilizó el algoritmo de Metrópolis, por lo que tampoco se usaron longitudes lineales más grandes que las citadas, ya que la precisión estadística se deteriora debido al enlentecimiento crítico del sistema. Por otro lado, incluso en sistemas puros (sin impurezas) las interfases fluctuantes son objetos muy lentos; en el sistema actual, la rugosidad que presenta la interfase debido al desorden congelado aleatorio hace que el equilibrio sea aún más difícil de alcanzar.

Se usaron típicamente $10^{7}$ pasos de Monte Carlo (MCS) por sitio de red para alcanzar el equilibrio, y luego se registraron promedios cada $2 \times 10^{7}$ MCS. Estos números claramente son bastante modestos, pero hay que recordar que es necesario realizar un promedio de más de 1000 configuraciones diferentes de las impurezas aleatorias para llevar a cabo el promedio $[\ldots]_{a v}$.

El observable que se midió fue la magnetización por espín

$$
m=\frac{1}{L M} \sum_{i=1}^{L M} S_{i},
$$


a partir de la cual se obtienen los momentos, $[\langle|m|\rangle]_{a v},\left[\left\langle m^{2}\right\rangle\right]_{a v} \mathrm{y}\left[\left\langle m^{4}\right\rangle\right]_{a v}$. Estos permiten construir el cumulante de cuarto orden

$$
U=1-\frac{\left[\left\langle\left|m^{4}\right|\right\rangle\right]_{a v}}{3\left[\left\langle\left|m^{2}\right|\right\rangle\right]_{a v}^{2}}
$$

\subsubsection{Resultados de las simulaciones}

Dado el resultado obtenido de la ecuación (6.3) $T_{c b}=2,18802$, se estudian dos temperaturas $T=1,50$ y $T=1,75$, variando la intensidad de los campos superficiales $\left|h_{s}\right|$. Es importante destacar que no se debe trabajar a temperaturas bastante bajas, ya que las barreras de energía libre que restringen las fluctuaciones interfaciales enlentecen demasiado la dinámica; por otro lado, tampoco se debe trabajar cerca de $T_{c b}$, para evitar un crossover con la criticalidad del bulk. Por lo tanto, las opciones $T=1,50$ y $T=1,75$ son aceptables entre estos dos requisitos. Se mostrarán primero los resultados para $T=1,75$.

Las figuras (6.1) y (6.2) muestran los resultados para $[\langle|m|\rangle]_{a v} \mathrm{y}\left[\left\langle m^{2}\right\rangle\right]_{a v}, v s h_{s}$ en los paneles superiores y $v s\left(h_{s}-h_{w}\right) M^{1 / 3}$ en los paneles inferiores. El valor estimado del campo superficial crítico $h_{w}(T) \simeq 0,640(6)$ se obtiene del punto de intersección de $[\langle|m|\rangle]_{a v}$ y $\left[\left\langle m^{2}\right\rangle\right]_{a v}$ (se pueden ver los gráficos amplificados en los insets de cada figura).

El hecho de que se produzcan dichos puntos de intersección es una clara evidencia de que $\beta=0$, es decir, se verifica la validez de la ecuación (6.22) sobre la (6.16). Dicho punto de intersección, cercano a $h_{s}=0,64$, también se observa en el cumulante $U$, presentado en el panel inferior izquierdo de la figura (6.3), sin embargo la precisión al momento de determinar el punto crítico disminuye debido a la suave dependencia de $U$ con respecto a $h_{s}$ como así también a la falta de estadística de la muestra. Los escaleos que se presentan en ambas figuras, los cuales utilizan la variable $\left(h_{s}-h_{w}\right) M^{1 / 3}$, tienen una calidad razonable, dada la precisión estadística moderada de las simulaciones, y por lo tanto implican que los datos son realmente compatibles con una variable de escala 

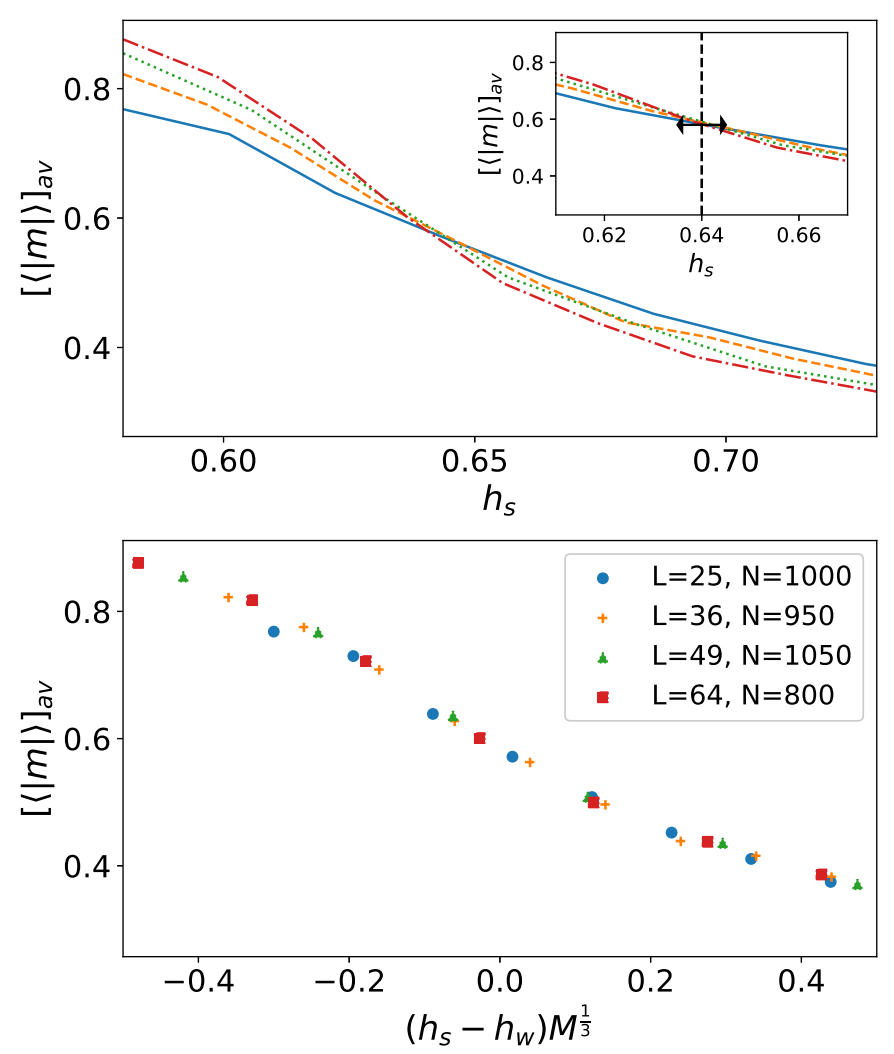

FIGURA 6.1. Gráfico del valor absoluto promedio de la magnetización $[\langle|m|\rangle]_{a v}$ vs $h_{s}$ (arriba). Se muestran los datos correspondientes a la temperatura $T=1,75$. Se indican el tamaño de las muestras $(L)$ y el número de promedios en diferentes configuraciones $(N)$. Todos los tamaños de muestra tienen la misma relación de aspecto generalizada $c=L^{3 / 2} / M=1$. Inset: Zoom de los datos mostrados en el panel superior, en el cual se puede observar con mayor precisión el punto de intersección común de los datos. La línea vertical (discontinua) muestra el punto de intersección de la magnetización medida para muestras de diferente tamaño, mientras que la línea horizontal (continua) da una estimación del error en la evaluación del punto crítico. Por último, en el panel inferior se muestra el gráfico de escala del valor absoluto de la magnetización $[\langle|m|\rangle]_{a v}$ vs $\left(h_{s}-h_{w}\right) M^{1 / 3}$. En este se observa el colapso de los datos, obtenido al tomar $\beta=0$ y $h_{w}=0,64$

$M / \xi_{\|} \propto M\left|h_{s}-h_{w}\right|^{3}$, como se esperaba. De esta manera, aunque falla la hiper escala, una forma restringida de escala de tamaño finito parece seguir siendo válida.

Por otro lado, los paneles superiores de dicha figura, presentan la susceptibilidad $T \chi^{\prime}=L M\left(\left[\left\langle m^{2}\right\rangle\right]_{a v}-[\langle|m|\rangle]_{a v}^{2}\right)$. Dado que en la transición de wetting, $[\langle|m|\rangle]_{a v} \mathrm{y}\left[\left\langle m^{2}\right\rangle\right]_{a v}$ 

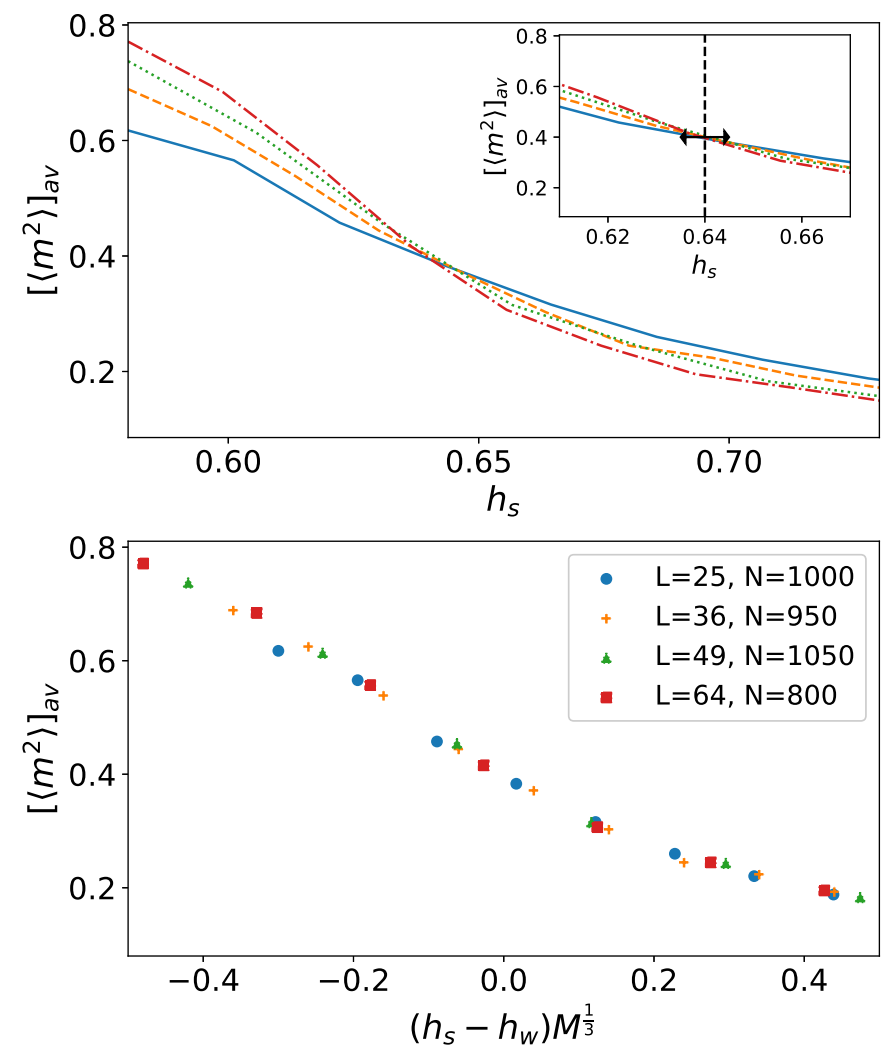

Figura 6.2. Gráfico del promedio de la magnetización al cuadrado $\left[\left\langle m^{2}\right\rangle\right]_{a v}$ $v s h_{s}$ (arriba). Se muestran los datos correspondientes a la temperatura $T=1,75$. Se indican el tamaño de las muestras $(L)$ y el número de promedios en diferentes configuraciones $(N)$. Todos los tamaños de muestra tienen la misma relación de aspecto generalizada $c=L^{3 / 2} / M=1$. Inset: Inset: Zoom de los datos mostrados en el panel superior, en el cual se puede observar con mayor precisión el punto de intersección común de los datos. La línea vertical (discontinua) muestra el punto de intersección de la magnetización medida para muestras de diferente tamaño, mientras que la línea horizontal (continua) da una estimación del error en la evaluación del punto crítico. Por último, en el panel inferior se muestra el gráfico de escala del promedio de la magnetización al cuadrado $\left[\left\langle m^{2}\right\rangle\right]_{a v}$ vs $\left(h_{s}-h_{w}\right) M^{1 / 3}$. En este se observa el colapso de los datos, obtenido al tomar $\beta=0$ y $h_{w}=0,64$

son, nada más ni nada menos, los puntos de intersección de las figuras (6.1) y (6.2), se podría esperar que $T \chi^{\prime} / L M$ también sean constantes finitas en los puntos de intersección, independientemente del tamaño. Sin embargo, el panel superior izquierdo de la figura (6.3) demuestra que no solo este no es el caso, sino que $T \chi^{\prime} / L M$ es muy pequeña, y 
disminuye sistemáticamente a medida que aumenta el tamaño, figura(6.4).
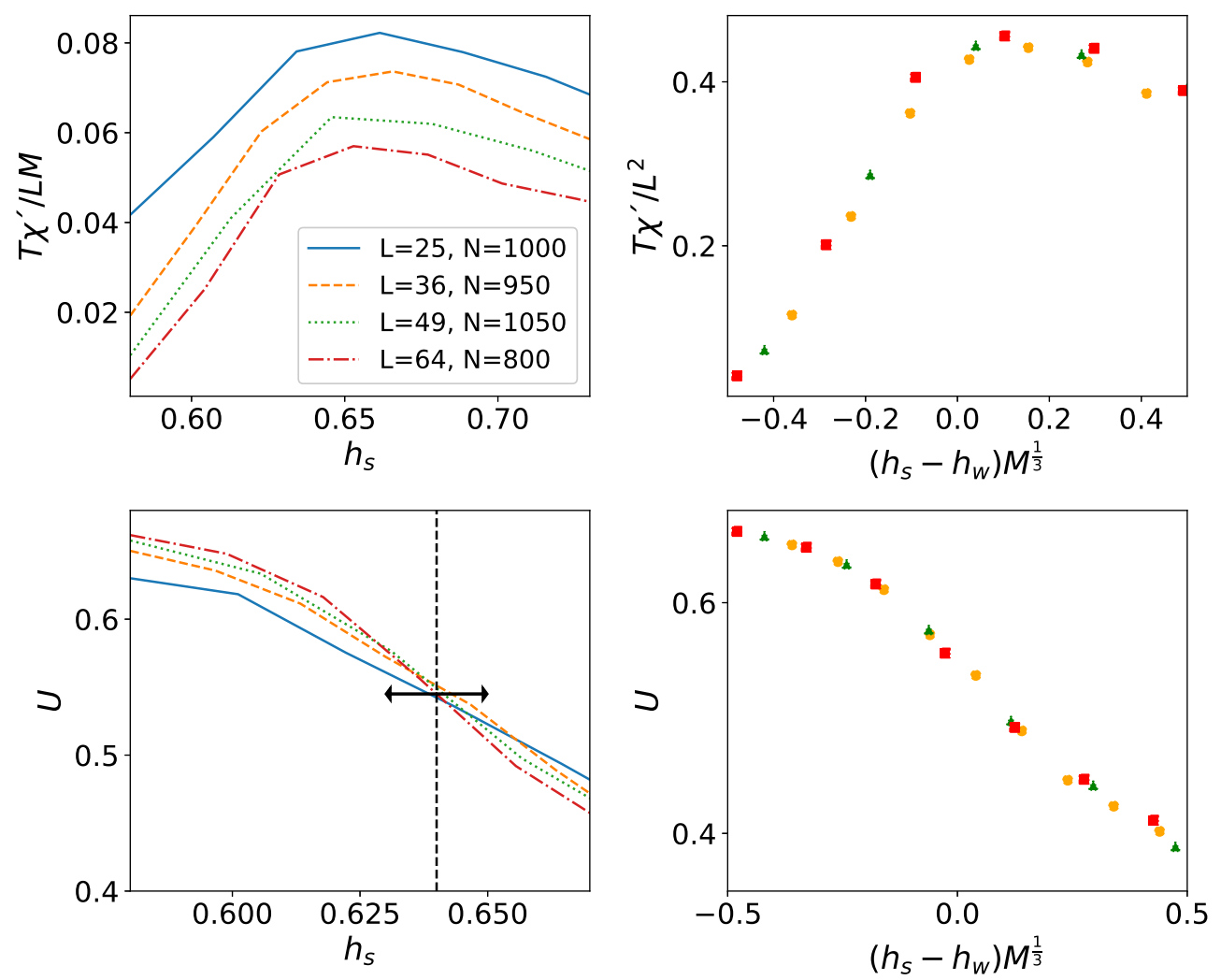

FigURA 6.3. Lo mismo que en las figuras anteriores pero para la susceptibilidad (paneles superiores) y el cumulante (paneles inferiores)

De hecho, teniendo en cuenta la ecuación (6.10), si $\beta=0$ y $\tilde{\chi}^{\prime}(c, 0)$ existiera, la conclusión $T \chi^{\prime} / L M=$ constante para $h_{s}=h_{w}(T)$, es inevitable. Por otro lado, si se cumple (6.8), la ecuación (6.12) con $\tilde{\chi^{\prime}}(c, 0)=$ constante y $\beta=0$, daría lugar a

$$
\left.k_{B} T \chi^{\prime}\right|_{h w} \propto M^{1+v_{\perp} / v_{\|}}=M^{5 / 3} \propto L^{5 / 2}
$$

mientras que las figuras (6.3) y (6.4) muestran evidencia numérica de

$$
\left.k_{B} T \chi^{\prime}\right|_{h w} \propto=M^{4 / 3} \propto L^{2} .
$$

Este resultado es claramente compatible con las ecuaciones (6.14) y (6.15). Por tal motivo se puede concluir, nuevamente, que $\gamma=4, \beta=0$, y que la forma restringida de escala 


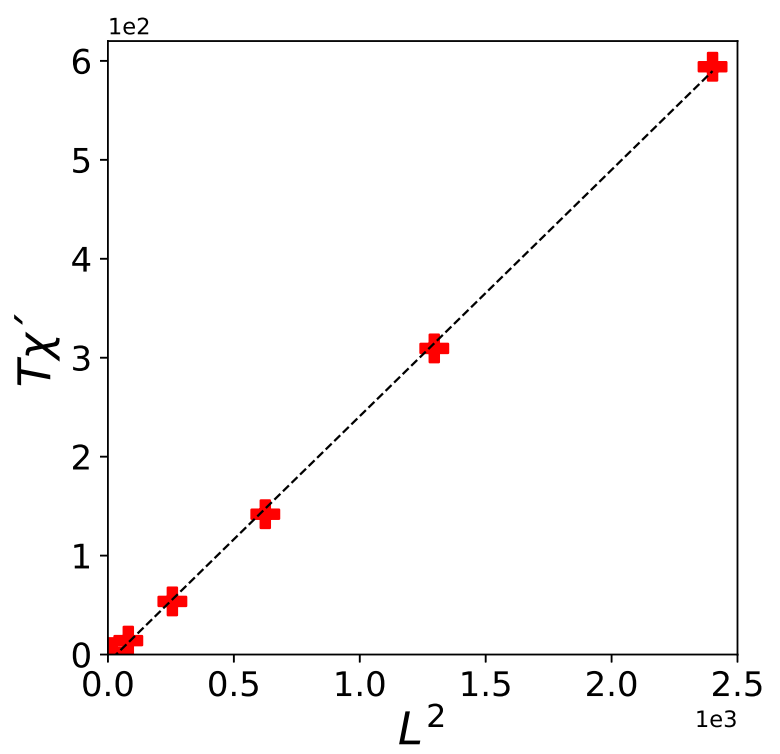

Figura 6.4. Gráfico de $T \chi^{\prime}$ vs $L^{\gamma / v_{\perp}}=L^{2}$ obtenido para $T=1,75$ usando los datos medidos en la transición de mojado. La línea recta se ha dibujado para guiar el ojo. Más detalles en el texto.

de tamaño finito (6.8) se mantiene válida con la función de escala $\tilde{P}\left(c, M / \xi_{\|}, m \xi_{\|}^{\beta / v_{\|}}\right)$, la cual tiene la propiedad que $\tilde{\chi}^{\prime}\left(c, M / \xi_{\|}\right)$desaparece, y el término de corrección principal en las ecuaciones (6.10) y (6.12), sobrevive y reemplaza el comportamiento mostrado en las ecuaciones (6.14) y (6.15).

Es importante notar que en el límite $M / \xi_{\|} \rightarrow 0$ el factor $\xi_{\|}^{-2 \beta / v_{\|}}$en la ecuación (6.10) se debe cancelar. Por lo tanto, asumiendo

$$
\tilde{\chi}\left(c, \frac{M}{\xi_{\|}} \rightarrow 0\right)=c t e \xi_{\|}^{2 \beta / v_{\|}} M^{-2 \beta / v_{\|}}
$$

se obtiene

$$
k_{B} T \chi^{\prime}=\operatorname{cte} L M^{1-2 \beta / v_{\|}}=c t e L^{1+v_{\|} / v_{\perp}-2 \beta / v_{\|}} c^{-\left(1-2 \beta / v_{\perp}\right)},
$$

es decir, $k_{B} T \chi^{\prime} \propto L^{5 / 2}$ si $\beta=0$. Sin embargo, es posible evitar una contradicción con el resultado correcto $k_{B} T \chi^{\prime} \propto L^{2}$ en la transición, cuando la constante anterior es igual a cero, es decir, cuando las funciones de escaleo $\tilde{m}_{1}\left(c, M / \xi_{\|}\right)$y $\tilde{m}_{2}\left(c, M / \xi_{\|}\right)$se comportan 
como:

$$
\tilde{m}_{2}\left(c, \frac{M}{\xi_{\|}} \rightarrow 0\right)=\left(\tilde{m}_{1}\left(c, \frac{M}{\xi_{\|}} \rightarrow 0\right)\right)^{2} .
$$

Como se ha demostrado en las figuras (6.1) y (6.2), existe evidencia numérica de que el primer y segundo momento de la magnetización exhiben el comportamiento propuesto en la nueva hipótesis de escala postulada con $M / \xi_{\|}$, o de manera equivalente $\operatorname{con}\left(h-h_{s}\right) M^{1 / 3}$.

La segunda temperatura $T=1,50$ (figuras (6.5), (6.6), (6.7), (6.8)) corrobora estos hallazgos, aunque la precisión es claramente menor.

Aquí, la transición se produce en un campo algo mayor $h_{w}(T) \simeq 0,74(7)$; (ver figuras (6.5) y (6.6)) y la variación con $h_{s}$ es más pronunciada, pero aún se observa una calidad razonable de escala contra la variable $\left(h_{s}-h_{w}\right) M^{1 / 3}$ (Figuras (6.5), (6.6) y (6.7)). Además, la relación $\left.\chi^{\prime}\right|_{h_{w}} \propto L^{2}$ también está confirmada (figura (6.8)). Es importante observar que las fluctuaciones de $T \chi^{\prime} / L M$ en los datos sin escala son más pronunciadas que la gráfica correspondiente para $T=1,75$ (figura (6.3)). Sin embargo, la figura (6.7) indica que la escala $T \chi^{\prime} / L^{2}$ versus $\left(h_{s}-h_{w}\right) M^{1 / 3}$ se mantiene razonablemente bien, pero los datos correspondientes a la red más grande $(L=64, M=512)$ no se han tenido en cuenta ya que están notablemente por debajo de la función de escala. Esto indica que el enlentecimiento crítico no se puede descuidar.

Resumiendo, los datos para $T=1,50$ en su conjunto confirman las conclusiones sobre la naturaleza de los fenómenos críticos en la transición de mojado, como se indicó anteriormente. 

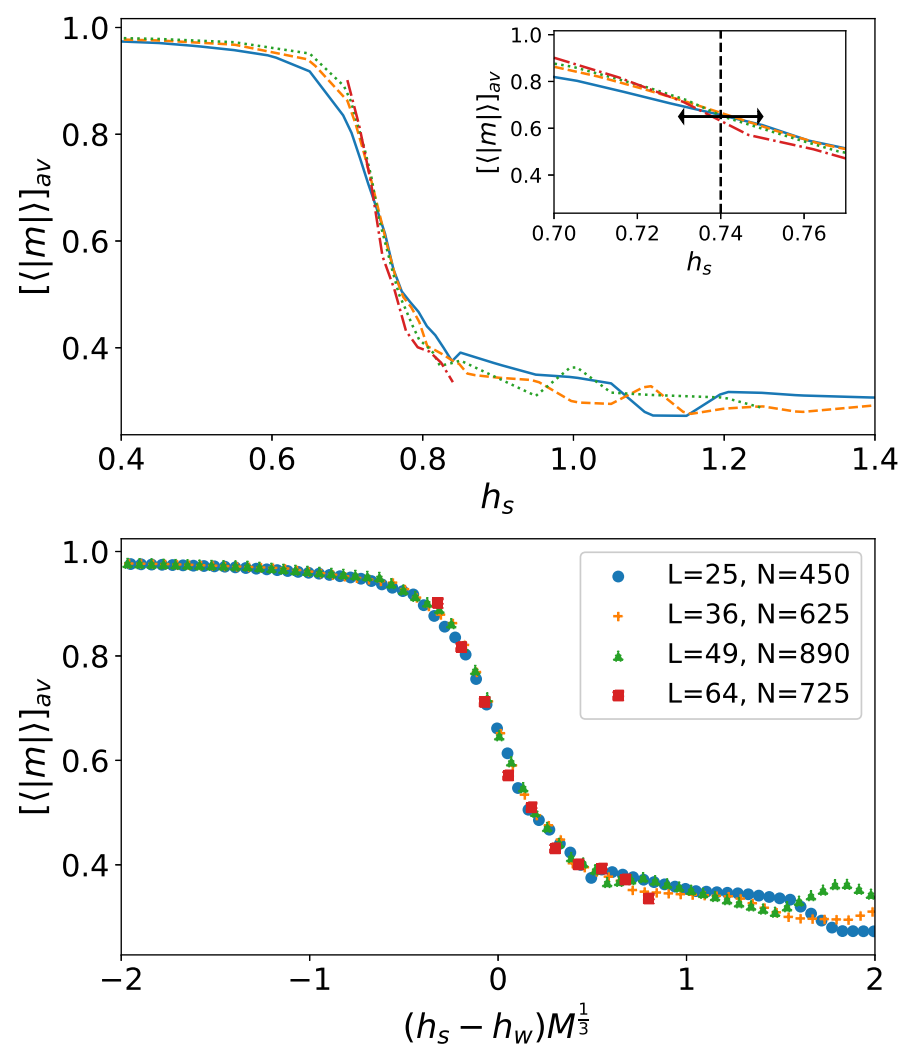

FIGURA 6.5. Gráfico del valor absoluto promedio de la magnetización $[\langle|m|\rangle]_{a v}$ vs $h_{s}$ (arriba). Se muestran los datos correspondientes a la temperatura $T=1,5$. Se indican el tamaño de las muestras $(L)$ y el número de promedios en diferentes configuraciones $(N)$. Todos los tamaños de muestra tienen la misma relación de aspecto generalizada $c=L^{3 / 2} / M=1$. Inset: Zoom de los datos mostrados en el panel superior, en el cual se puede observar con mayor precisión el punto de intersección común de los datos. La línea vertical (discontinua) muestra el punto de intersección de la magnetización medida para muestras de diferente tamaño, mientras que la línea horizontal (continua) da una estimación del error en la evaluación del punto crítico. Por último, en el panel inferior se muestra el gráfico de escala del valor absoluto de la magnetización $[\langle|m|\rangle]_{a v}$ vs $\left(h_{s}-h_{w}\right) M^{1 / 3}$. En este se observa el colapso de los datos, obtenido al tomar $\beta=0$ y $h_{w}=0,74$ 

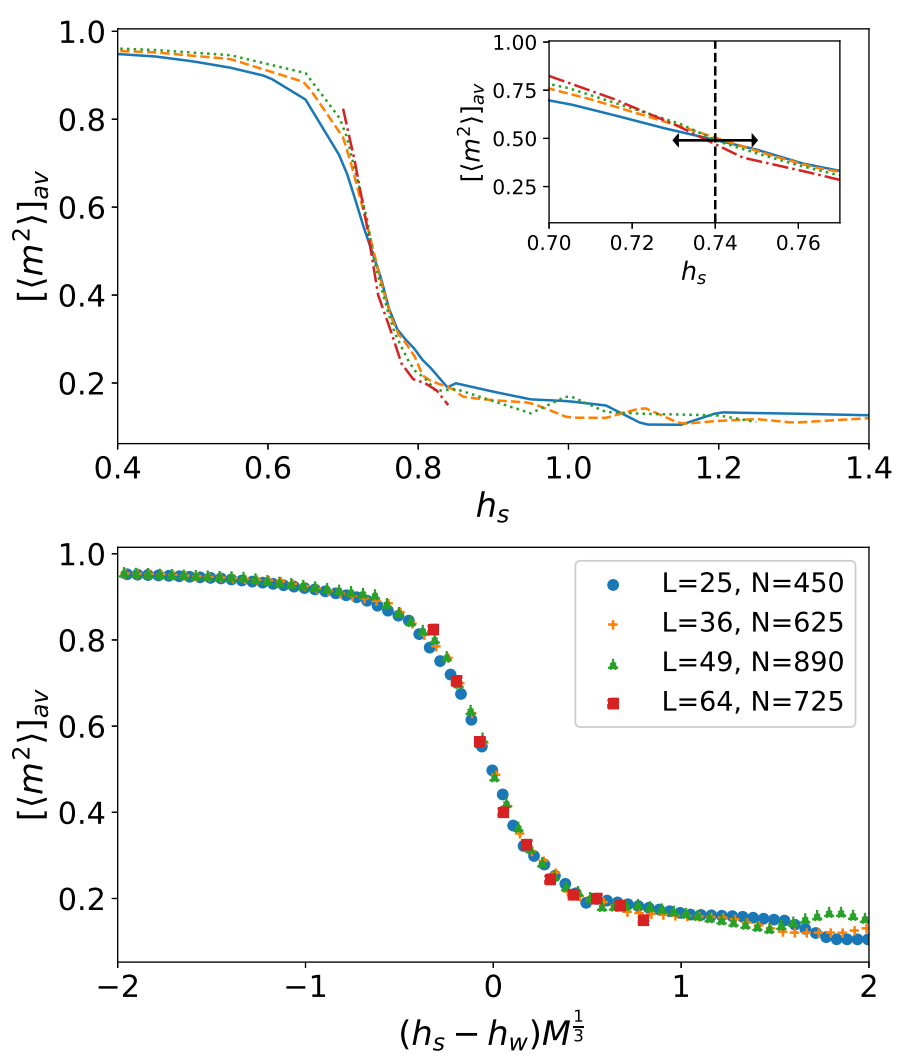

FiguRA 6.6. Gráfico del promedio de la magnetización al cuadrado $\left[\left\langle m^{2}\right\rangle\right]_{a v}$ vs $h_{s}$ (arriba). Se muestran los datos correspondientes a la temperatura $T=1,5$. Se indican el tamaño de las muestras $(L)$ y el número de promedios en diferentes configuraciones $(N)$. Todos los tamaños de muestra tienen la misma relación de aspecto generalizada $c=L^{3 / 2} / M=1$. Inset: Zoom de los datos mostrados en el panel superior, en el cual se puede observar con mayor precisión el punto de intersección común de los datos. La línea vertical (discontinua) muestra el punto de intersección de la magnetización medida para muestras de diferente tamaño, mientras que la línea horizontal (continua) da una estimación del error en la evaluación del punto crítico. Por último, en el panel inferior se muestra el gráfico de escala del promedio de la magnetización al cuadrado $\left[\left\langle m^{2}\right\rangle\right]_{a v}$ vs $\left(h_{s}-h_{w}\right) M^{1 / 3}$. En este se observa el colapso de los datos, obtenido al tomar $\beta=0$ y $h_{w}=0,74$ 

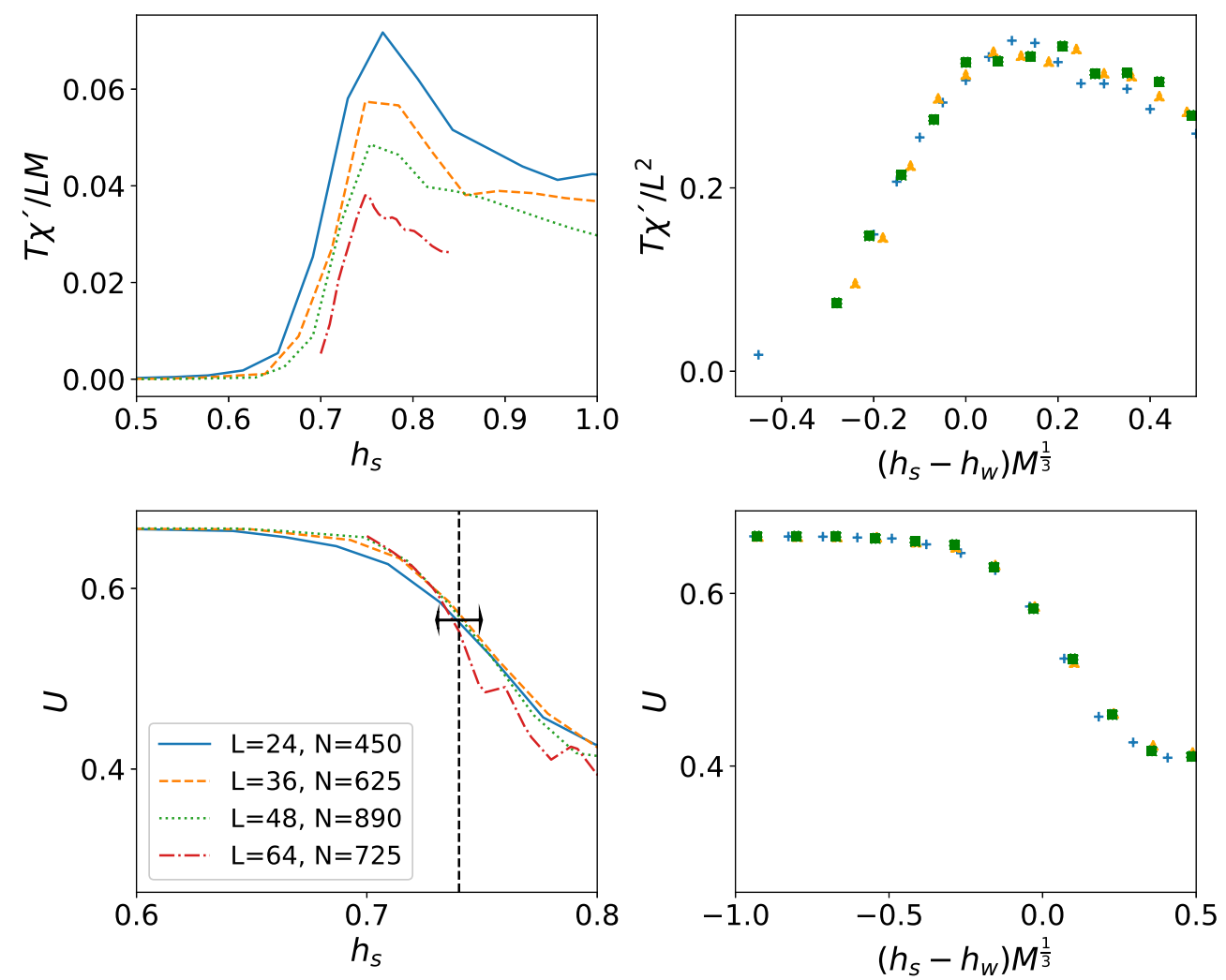

FiguRA 6.7. Lo mismo que en las figuras anteriores pero para la susceptibilidad (paneles superiores) y el cumulante (paneles inferiores) 


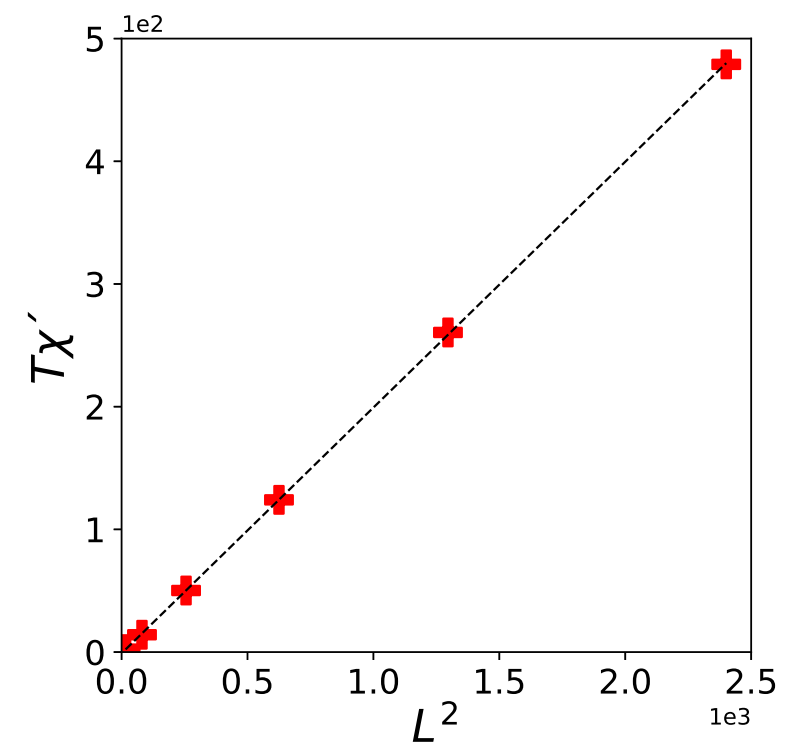

FiguRA 6.8. Gráfico de $T \chi^{\prime}$ vs $L^{\gamma / v_{\perp}}=L^{2}$ obtenido para $T=1,5$ usando los datos medidos en la transición de mojado. La línea recta se ha dibujado para guiar el ojo. Más detalles en el texto. 



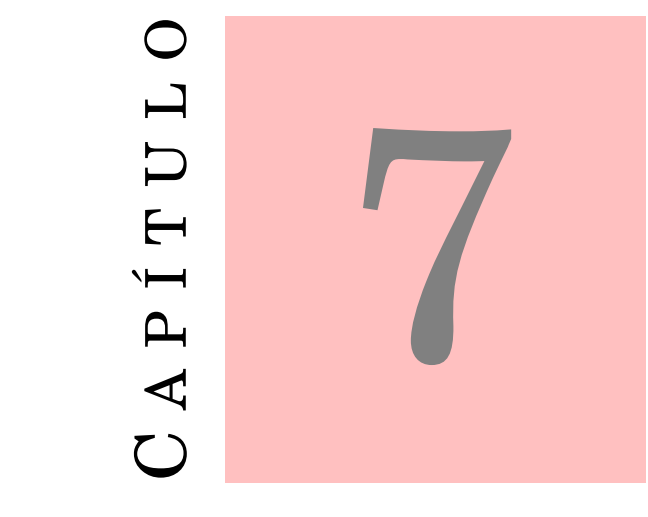

\section{CONCLUSiOneS}

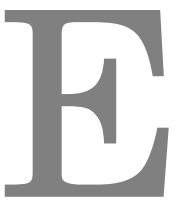

$\mathrm{n}$ esta tesis se han estudiado distintos aspectos del comportamiento de sistemas confinados en el marco de la Mecánica Estadística empleando, como herramienta fundamental, simulaciones computacionales de tipo Monte Carlo.

Como se mencionó en la discusión del capítulo 2, luego de trabajar con distintos tipos de algoritmos, como ser Metrópolis y Wang-Landau en su implementación propuesta por Belardinelli y Pereyra, se han encontrado ventajas y desventajas de los mismos.

El algoritmo de Wang-Landau permite obtener una estimación confiable de la densidad de estados de un sistema, de manera precisa y eficiente, en función de los parámetros que resulten adecuados para los sistemas que se desean estudiar. A partir de la densidad de estados del modelo se puede construir la función de partición y calcular las cantidades termodinámicas de interés, en rangos extensos de temperatura. Por otro lado, la independencia de la temperatura y la secuencia de pasos que conforma el algoritmo 
impide que este caiga en los mínimos de energía locales que pueda presentar el espacio de fases del modelo. La penalización de los estados más probables permite que todos los niveles del sistema sean visitados, incluso aquellos con menos probabilidad. Sin embargo, la formulación de la densidad de estados puede provocar que la convergencia sea larga, y en ocasiones pueda no estar asegurada. A medida que se agregan parámetros, la convergencia se vuelve más difícil ya que se trabaja en espacios dimensionales más grandes. Esto requiere más tiempo de cálculo. Por tales motivos, a lo largo de este trabajo hubo casos en los que el uso del algoritmo de WL no presentaba ventaja alguna sobre el algoritmo de Metrópolis.

En el capítulo 4 se logró caracterizar el modelo de BEG, mediante la construcción de su diagrama de fases. Este incluía líneas de transición de primer y segundo orden, las cuales delimitaban las distintas fases: paramagnética, ferromagnética y cuadrupolar. Para lograr dicha caracterización, primero fue necesario realizar un análisis exhaustivo del diagrama de fases completo. El parámetro $D$, que controla la densidad de vacancias, permitió llevar al sistema desde un Ising simple $(D \rightarrow-\infty)$, donde todos los sitios están ocupados por espines $( \pm 1)$, hasta el caso en el que se llena de vacancias. Dicha tarea se llevó a cabo usando el algoritmo de WL, sin embargo, esto no fue posible en la fase cuadrupolar, la cual presenta un arreglo de vacancias y espines a modo de tablero de ajedrez. Al estar rodeados de vacancias los espines no tienen interacciones mutuas, de modo que el estado es paramagnético. Por tal motivo, hubo que recurrir al uso de un nuevo parámetro de orden, el cual requería separar la red en dos subredes y, por lo tanto, duplicar los parámetros de la densidad de estados. Esto provocó que los tiempos de convergencia crecieran y no fuera conveniente el uso de WL.

Para el caso de $\alpha=0$, en que el modelo BEG se reduce al modelo $\mathrm{BC}$, se verificó que la línea de transición se convierte de segundo a primer orden en un punto tricrítico. Dichos puntos tricríticos fueron también calculados para $\alpha \neq 0$, completando así el diagrama de 
fases.

Por otro lado, se estudió el modelo de BEG con interacciones a segundos vecinos. Esta interacción favorece el orden del sistema cuando es tomada de forma ferromagnética, por lo que se encontró una nueva fase ausente en el diagrama del modelo de BEG con interacciones a primeros vecinos, llamada cuadrupolar ferromagnética. En dicha fase, la subred en la que se encontraban los espines \pm 1 de la fase cuadrupolar, ahora presenta orden ferromagnético.

Finalmente, se determinaron las temperaturas críticas en el límite termodinámico como así también se confirmó que la clase de universalidad del modelo corresponde a la de Ising $2 D$.

En el capítulo 5 se estudió el modelo BEG bajo los efectos del confinamiento y se consideró el escaleo de tamaño finito para el estudio de transiciones de mojado. Se utilizó el procedimiento propuesto por Albano y Binder [27] en el que las longitudes lineales $L$ y $M$ del film $L \times M$ varían de modo tal que la relación de aspecto generalizada $c$ se mantiene constante. Esto permite analizar los datos en completa analogía con el estudio de las transiciones de fase del bulk, donde se usan campos de superficie antisimétricos $\left(h_{1}=-h_{L}\right)$. De esta manera, la magnetización total $m$ del sistema actúa como un parámetro de orden, pero el exponente crítico apropiado es $\beta=0$. Si bien dicho procedimiento es válido para el modelo de Ising y el BC, en este trabajo se probó su validez para el modelo de BEG.

Se han determinado líneas de transición de mojado para distintos valores de $h_{s}, D$ y $\alpha$. Para estimar la $T_{w}$, el segundo momento de la magnetización y el cumulante de Binder, resultaron ser muy útiles, mostrando solo efectos débiles de tamaño finito. Se mostró que a medida que $h_{s}$ aumenta, la $T_{w}$ disminuye, mientras que cuando $h_{s} \rightarrow 0$, $T_{w} \rightarrow T_{b}$. Por otro lado, la presencia de impurezas no magnéticas (vacancias) pueden favorecer la transición de mojado dependiendo de $D$ y de $\alpha$. Finalmente se utilizó el 
método de integración termodinámica para verificar las transiciones de segundo orden. Éste método es muy útil al momento de trabajar con transiciones de primer orden, en las cuales no es posible realizar un escaleo de tamaño finito, sin embargo, todas las transiciones que se encontraron fueron de segundo orden.

También se estudió la adsorción de las vacancias en la interfase entre las regiones ricas en espines -1 y 1 . Se mostró que la adsorción, crece rápidamente cerca de $T_{w}$, asociada, posiblemente, con una singularidad en la derivada de $W_{0}$ con respecto a la temperatura. Por otro lado, si se mantiene $D=$ cte y se aumenta $\alpha$, el sistema necesitará mayor temperatura para alcanzar una transición de mojado. Éste método permite localizar las transiciones de mojado y de bulk de forma precisa y sencilla.

Finalmente, los resultados conocidos de manera exacta para el modelo de Ising en la red cuadrada, el cual es el límite del modelo para $D \rightarrow-\infty$, y los trabajos realizados para el modelo de $\mathrm{BC}(\alpha=0)$, permitieron mostrar que los resultados obtenidos en este trabajo se verifican muy bien.

Por último, en el capítulo 6 se estudiaron los efectos del confinamiento sobre el modelo RBIM. La diferencia principal de éste con el modelo BEG es que el ruido está congelado, es decir, las impurezas, causadas por la aleatoriedad de la constante de acoplamiento, se encuentran fijas en la red. Esto provoca que la transición de deslocalización de la interfase se vea afectada, y por lo tanto sus exponentes críticos, causando así una violación en la hiper escala. En éste capítulo no solo se demostró la violación de hiper escala para el wetting crítico, sino que se propuso un método de escaleo de tamaño finito sin hiper escala y se corroboró esta nueva hipótesis mediante simulaciones computacionales.

Durante el desarrollo de la presente Tesis Doctoral fueron publicados los resultados expuestos en los capítulos 4 y 6, en las referencias [55] y [56] respectivamente, y se encuentra en preparación otro trabajo relativo a los estudios detallados en el capítulo 5. 


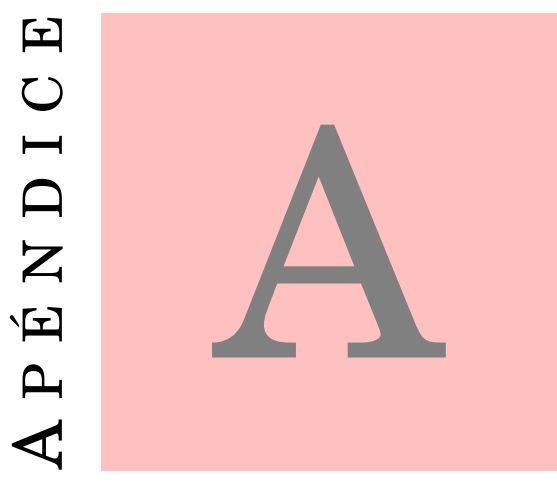

\section{OBTENCIÓN DEL PARÁMETRO DE}

\section{ORDEN PARA EL PUNTO TRICRÍTICO}

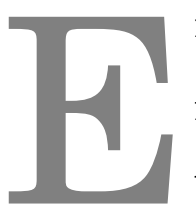

n el capítulo 3 se comentó que la hipótesis de escala para puntos tricríticos se formula mejor en términos de los campos de escala $\tau$ y $\eta$, los cuales son combinaciones lineales de los campos termodinámicos. Estos están determinadas, en parte, por la geometría del diagrama de fases, y desaparecen en el punto tricrítico. En éste apéndice se hará un análisis detallado de cómo obtener éstos campos de escala, a partir de los cuales se puede obtener un nuevo parámetro de orden para caracterizar los puntos tricríticos.

Para ello, se considera un diagrama como el de la figura(A.1). Las líneas críticas $L_{1}$ (línea punteada) y $L_{2}$ (línea llena) separan la fase ordenada de la desordenada. En 


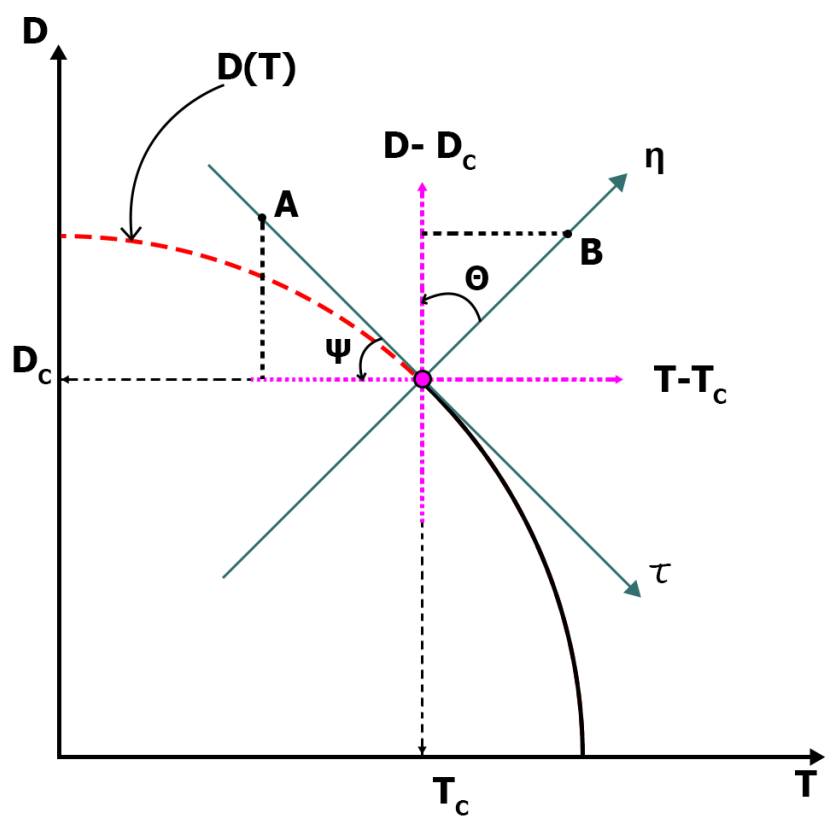

Figura A.1. Representación de la línea de transición de primer orden $D(T)$ en el plano $H^{+}=0$. $\left(T_{c}, D_{c}\right)$ es, en general, un punto tricrítico cuando se tiene una línea de transición de segundo orden. $\tau$ y $\eta$ son las direcciones de los campos de mezcla. Todos los puntos $(T, D)$ en la dirección $\tau$ (como el punto $A$ ) tienen $\eta=0$, mientras que los puntos como $B$ tienen $\tau=0$

general, cerca del punto crítico, la ecuación de la línea $L_{2}$ se puede expandir como

$$
D_{2} T=D_{c}-r\left(T-T_{c}\right)+b_{2}\left(T-T_{c}\right)^{\phi_{2}}+\cdots
$$

para $T>T_{c}$. El exponente de corrimiento $\Phi$, describe la forma de la línea crítica y, usualmente, satisface la condición $1<\Phi \leq 2$. Se puede proceder de forma análoga con la línea $L_{1}$

$$
D_{1} T=D_{c}-r\left(T-T_{c}\right)+b_{1}\left(T-T_{t}\right)^{\phi_{1}}+\cdots,
$$

para $T \leq T_{c}$. La aparición del mismo coeficiente

$$
r=\left(\frac{\partial D_{1}}{\partial T}\right)_{T_{c}}=\left(\frac{\partial D_{2}}{\partial T}\right)_{T_{c}}=\tan \Psi
$$

en el término lineal de (A.1) y (A.2), es una evidencia de la continuidad de la pendiente 
en el punto tricrítico. Por lo tanto, obviando los términos superiores, se puede escribir:

$$
\begin{aligned}
& D(T)=D_{c}-r\left(T-T_{c}\right) \\
& \left(D(T)-D_{c}\right)+r\left(T-T_{c}\right)=0 .
\end{aligned}
$$

esta ecuación representa la línea recta tangente a la curva $D(T)$ en el punto $\left(T_{c}, D_{c}\right)$, por lo tanto, se puede definir el primer campo de escala

$$
\eta=\left(D(T)-D_{c}\right)+r\left(T-T_{c}\right)
$$

el cual, como se mencionó previamente, puede tener cualquier dirección no paralela a la línea tangente. De forma similar se puede definir

$$
\tau=\left(T-T_{c}\right)+s\left(D(T)-D_{c}\right)
$$

donde $s=\tan (\Theta) . \eta=0$ define el eje $\tau$, mientras que $\tau=0$ define el eje $\eta$. Cuando $s=r=0$ se recupera la simetría $\tau=\left(T-T_{c}\right)$ y $\eta=\left(D(T)-D_{c}\right)$ (Modelo de Ising).

Finalmente, la parte singular de la energía libre se puede escribir en función de los nuevos campos de escala como

$$
f_{s}(\tau, D)=\tau^{2-\alpha_{t}} f^{ \pm}\left(\frac{\eta}{\tau^{\beta_{t} \delta_{t}}}\right)
$$

donde $(\tau, D) \rightarrow 0$ y \pm representa el signo de $\tau$. El paso siguiente en la determinación del punto tricrítico, y su clase de universalidad, será analizar el comportamiento de un parámetro de orden $m$, y su función de distribución de probabilidad

$$
P_{L}(m)=b L^{\beta / v} P^{*}\left(b L^{\beta / v} m\right)
$$

donde $v$ es el exponente crítico de la longitud de correlación, $b$ es una constante métrica no universal y $P^{*}$ es una función universal que permite obtener información de la clase de universalidad del punto tricrítico. Para ello se considera el Hamiltoniano del modelo BEG (capítulo 4)

$$
\mathscr{H}_{B E G}=-J \sum_{\langle i, j\rangle} s_{i} s_{j}+D \sum_{i=1}^{N} s_{i}^{2}-\alpha J \sum_{\langle i, j\rangle} s_{i}^{2} s_{j}^{2}
$$


cuya función de partición se puede escribir como

$$
\begin{aligned}
Z & =\sum_{\text {estados} \ell} \exp \left[\beta J \sum_{\langle i, j,\rangle} s_{i} s_{j}-\beta D \sum_{1}^{N} s_{i}^{2}+\beta \alpha J \sum_{\langle i, j,\rangle} s_{i}^{2} s_{j}^{2}\right] \\
& =\sum_{\text {estados } \ell} \exp \left[\tilde{t} E_{\ell}+\tilde{q} \mathscr{N}_{\ell}\right],
\end{aligned}
$$

donde $\tilde{t}=\beta J$ y $\tilde{q}=\beta D$ son la temperatura y el campo cristalino reducidos respectivamente, $\mathrm{y}$

$$
E_{\ell}=\sum_{\langle i, j\rangle} s_{i} s_{j}+\alpha \sum_{\langle i, j\rangle} s_{i}^{2} s_{j}^{2}, \quad \mathscr{N}_{\ell}=-\sum_{1}^{N} s_{i}^{2},
$$

a partir de los cuales se obtienen los valores medios

$$
\langle E\rangle=\frac{q}{Z} \frac{\partial \mathscr{Z}}{\partial \tilde{t}}, \quad\langle\mathscr{N}\rangle=\frac{q}{Z} \frac{\partial \mathscr{Z}}{\partial \tilde{q}}
$$

Si se toman $\hat{t}=\tilde{t}-\tilde{t}_{c}$ y $\hat{\omega}=\tilde{q}-\tilde{q}_{c}$, las ecuaciones (A.5) y (A.6) se pueden escribir de forma general tal que

$$
\eta=\hat{\omega}+r \hat{t}, \quad \tau=\hat{t}+s \hat{\omega} .
$$

Es importante destacar que, en las proximidades del punto tricrítico $\left(T_{c}, D_{c}\right)$, lo ejes ya no están dados por $\left(T-T_{c}\right)$ y $\left(D-D_{c}\right)$, sino por

$$
\begin{aligned}
& \hat{t}=\tilde{t}-\tilde{t}_{c}=\beta J-\beta_{c} J=J\left[\frac{1}{k_{B} T}-\frac{1}{k_{B} T_{c}}\right]=\frac{J}{k_{B} T}\left[\frac{T_{c}-T}{T_{c}}\right] \\
& \hat{\omega}=\tilde{q}-\tilde{q}_{c}=\beta D-\beta D_{c}=\frac{1}{k_{B} T_{c}}\left(D-D_{c}\right),
\end{aligned}
$$

los cuales difieren de los anteriores por un factor métrico.

Lo siguiente será ver cómo se relacionan las cantidades $E$ y $\mathscr{N}$ con los campos $\eta$ y $\tau$ que no están presentes de forma explícita en el Hamiltoniano. Para ello, se definen

$$
\begin{aligned}
& \langle\mathbb{E}\rangle=\frac{1}{\mathfrak{Z}} \frac{\partial \mathfrak{Z}}{\partial \tau}=\frac{1}{\mathfrak{Z}}\left(\frac{\partial \mathfrak{Z}}{\partial \hat{t}} \frac{\partial \hat{t}}{\partial \tau}+\frac{\partial \mathfrak{Z}}{\partial \hat{\omega}} \frac{\partial \hat{\omega}}{\partial \tau}\right), \\
& \langle\mathbb{N}\rangle=\frac{1}{\mathfrak{Z}} \frac{\partial \mathfrak{Z}}{\partial \eta}=\frac{1}{\mathfrak{Z}}\left(\frac{\partial \mathfrak{Z}}{\partial \hat{t}} \frac{\partial \hat{t}}{\partial \eta}+\frac{\partial \mathfrak{Z}}{\partial \hat{\omega}} \frac{\partial \hat{\omega}}{\partial \eta}\right) .
\end{aligned}
$$


Si se invierten las ecuaciones (A.13), se pueden escribir $\hat{t}$ y $\hat{q}$ en función de $\tau$ y $\eta$, tal que

$$
\hat{t}=\frac{1}{1-r s}(\tau-s \eta), \quad \hat{\omega}=\frac{1}{1-r s}(\eta-r \tau),
$$

cuyas derivadas con respecto a los campos de escala son

$$
\begin{array}{ll}
\frac{\partial \hat{\omega}}{\partial \tau}=-r\left(\frac{1}{1-r s}\right), & \frac{\partial \hat{\omega}}{\partial \eta}=\frac{1}{1-r s} \\
\frac{\partial \hat{t}}{\partial \tau}=\frac{1}{1-r s}, & \frac{\partial \hat{t}}{\partial \eta}=-s\left(\frac{1}{1-r s}\right) .
\end{array}
$$

Y por último, teniendo en cuenta que las derivadas parciales con respecto a $\tilde{t}$ y $\tilde{q}$ de (A.12), son iguales a las de (A.17) con respecto a $\hat{t}$ y $\hat{\omega}$

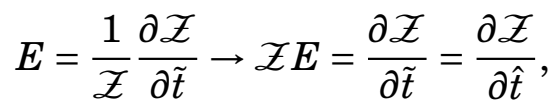

$$
\begin{aligned}
& \mathscr{N}=\frac{1}{Z} \frac{\partial \mathfrak{Z}}{\partial \tilde{q}} \rightarrow \mathfrak{Z} \mathscr{N}=\frac{\partial \mathscr{Z}}{\partial \tilde{q}}=\frac{\partial \mathfrak{Z}}{\partial \hat{\omega}} .
\end{aligned}
$$

Reemplazando en (A.17), se obtienen los parámetros de orden

$$
\begin{aligned}
& \mathbb{E}=\left(\frac{1}{1-r s}\right)(E-r \mathscr{N}), \\
& \mathbb{N}=\left(\frac{1}{1-r s}\right)(\mathscr{N}-s E) .
\end{aligned}
$$

Con los parámetros de orden en mano solo resta calcular la FDP. Pero antes de pasar a eso, es importante recordar un último concepto.

Sea $x_{i}$ una variable discreta con probabilidad de normalización $P\left(x_{i}\right)$ donde

$$
\langle x\rangle=\sum_{i} x_{i} P x_{i}
$$

Además

$$
\langle a x\rangle=\sum_{i} a x_{i} P x_{i}=a\langle x\rangle .
$$

Por otro lado, para una variable $y_{i}=a x_{i}$, existe una distribución de la probabilidad $P\left(y_{i}\right)$ tal que

$$
\langle y\rangle=\sum_{i} a x_{i} P^{\prime} a x_{i}=a\langle x\rangle
$$


APÉNDICE A. OBTENCIÓN DEL PARÁMETRO DE ORDEN PARA EL PUNTO TRICRÍTICO

al comparar las ecuaciones (A.26) y (A.27), se tiene que $P^{\prime}\left(a x_{i}\right)=P\left(x_{i}\right)$. Esto quiere decir que una constante no afectará el cálculo de la FDP de una variable. Por consiguiente se puede reducir el cálculo de la FDP $P(\mathbb{Q})$ de $(\mathrm{A} .23)$ al de la FDP $P(\mathscr{D})$, donde $\mathscr{D}=\mathscr{N}-s E$. 


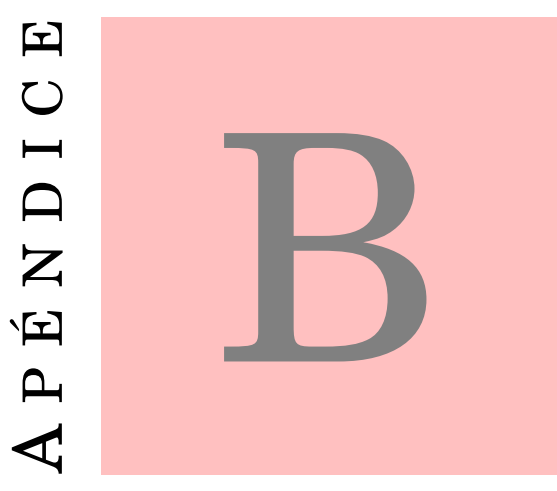

\section{MODELO BEG: OBTENCIÓN DEL}

\section{HAMILTONIANO}

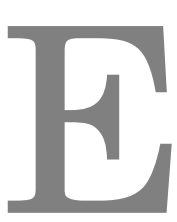

l interés por estudiar la mezcla $H e^{3}-H e^{4}$ surgió ya que, de los dos isotopos que presenta el Helio, el $\mathrm{He}^{4}$ abunda en la naturaleza, representando casi la totalidad del Helio disponible. Por tal motivo, las propiedades asignadas al Helio natural se refieren exclusivamente al $\mathrm{He}^{4}$, algunas de las cuales son:

- Presenta anomalías en la constante dieléctrica y en el calor específico a $2,2 K$

- La ebullición se producía de forma vigorosa por encima de $2,2 K$ y de forma suave y uniforme por debajo de la misma.

- La viscosidad se mantenía finita y dependiente de la temperatura por encima de $2,2 K$, mientras que descendía hasta hacerse despreciable por debajo ésta. A partir 
de ahí surge el nombre de "super fluido" que recibe el Helio para temperaturas menores a $2,2 K$.

- La conductividad calorífica crece considerablemente por debajo de $2,2 K$.

Este conjunto de hechos sugirió la existencia de dos fases en el Helio líquido que se llamaron $\mathrm{HeI}$ y $\mathrm{HeII}$ que son estables a temperaturas superiores e inferiores a $2,2 \mathrm{~K}$ respectivamente.

Por otro lado, una de las propiedades más destacadas del isotopo $H e^{3}$, es el mínimo que presenta su curva de fusión a $0,32 K$ y la ausencia de cambios significativos de los volúmenes relativos del sólido y del líquido a lo largo de toda esa curva, figura(B.1). Esto significa que para temperaturas menores a $0,32 K$, el sólido debe tener una entropía superior a la del líquido y por tal motivo, el calor de fusión debería hacerse negativo. Cuando la temperatura tiende a cero, las entropías de sólido y líquido deben volver a igualarse.
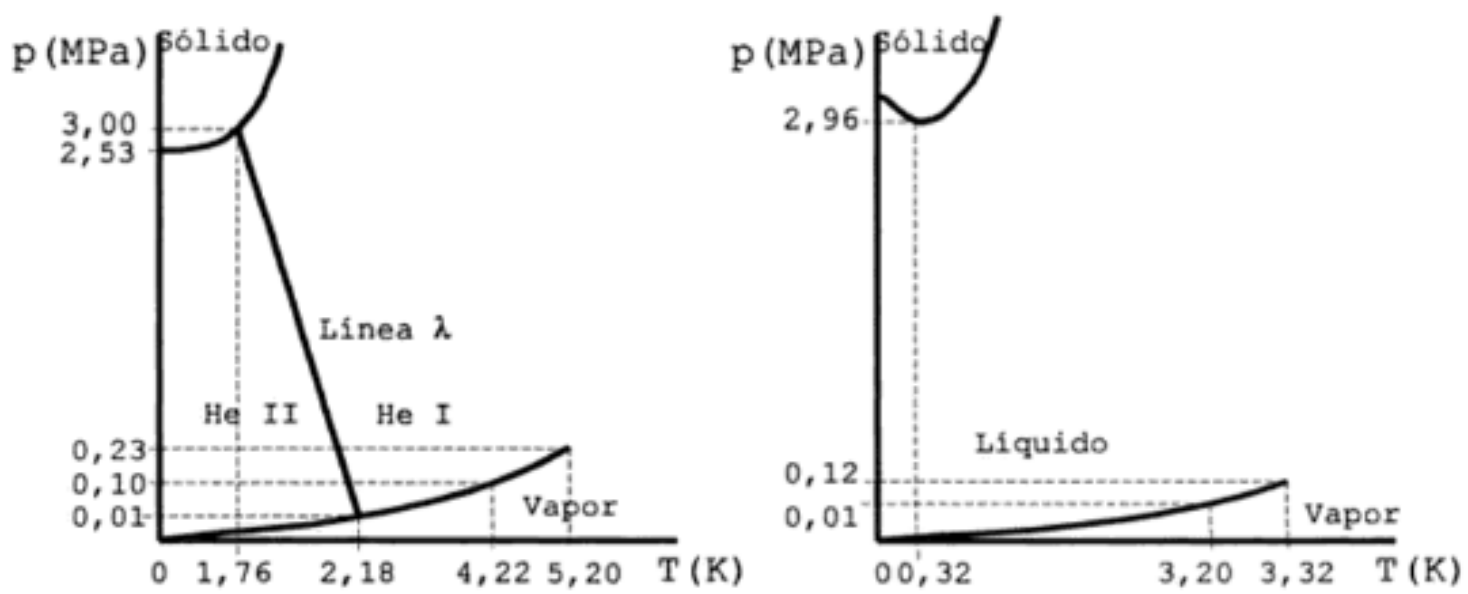

FiguRA B.1. Diagramas de fases en función de la presión y la temperatura para el $\mathrm{He}^{3}$ (izquierda) y el $\mathrm{He}^{4}$ (derecha).

Inicialmente no se encontraron transiciones a super fluido en el $H e^{3}$, sin embargo, con el avance de la tecnología, se pudieron realizar medidas más precisas y a temperaturas 
más bajas, las cuales permitieron encontrar dos fases superfluidas, $A$ y $B$, en ausencia de campo magnético, figura(B.2). La primera, $A$, es una fase fuertemente anisótropa, que define en el líquido un eje de simetría, de modo que todas sus propiedades macroscópicas modifican sus valores respecto a ese eje. La fase $B$ es básicamente isotrópica y posee un menor campo de existencia. La transición entre el $H e^{3}$ fluido y el super fluido es de segundo orden, mientras que la transición $A-B$ es de primer orden.

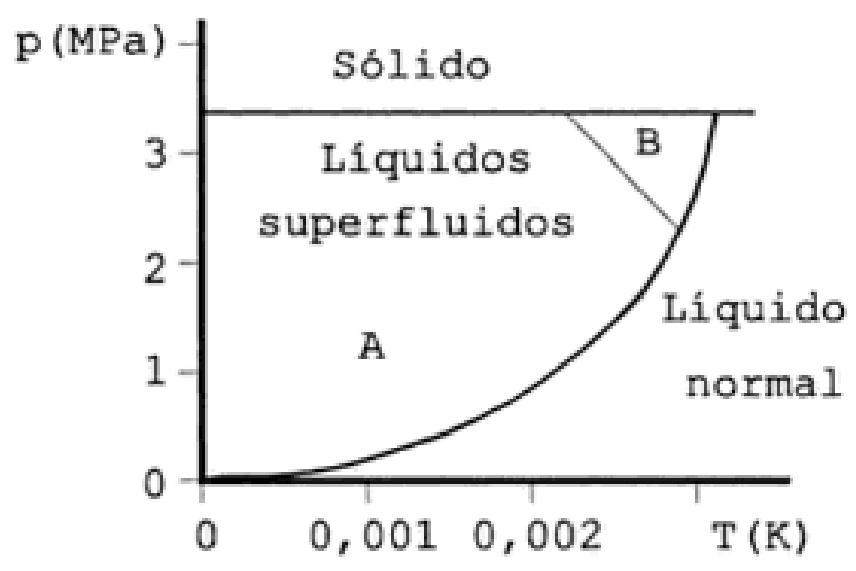

Figura B.2. Diagramas de fases en función de la presión y la temperatura para el $H e^{3}$, el cual presenta una transición de super fluido.

Al mezclar los dos fluidos, el $H e^{4}$ (super fluido) actúa como base del $H e^{3}$ (fluido) permitiendo ver fenómenos como separaciones de fases. Pero dependiendo de la concentración de $H e^{3}$ y $H e^{4}$, la mezcla es capaz de soportar la transición $\lambda$ o no. Por ejemplo, para una concentración de $H e^{3}$ menor al $67 \%$ la mezcla es capaz de experimentar una transición $\lambda$, mientras que para concentraciones mayores sufre una transición de primer orden a dos fases de las cuales, solo la fase de $\mathrm{He}^{4}$ será un super fluido.

El modelo de BEG, el cual permite reproducir de forma cualitativa, las características principales de los diagramas de fase para el super fluido y la transición de la separación de las fases, consiste en una red discreta y una variable de espín $s_{i}$, la cual puede tomar valores $1,-1$ y 0 , asociada a cada sitio de la red. Un átomo de $H e^{3}$ corresponde a $s_{i}=0$ 
$\mathrm{y}$ un átomo de $H e^{4}$ corresponde a $s_{i}= \pm 1$. Solo puede haber un átomo por sitio y no se permiten los sitios vacíos. El signo de $s_{i}$ asociado al átomo $\mathrm{He}^{4}$ se introduce con el propósito de proveer un "parámetro de orden"

$$
m=\frac{1}{N} \sum_{i=1}^{N}\left\langle s_{i}\right\rangle .
$$

Por otro lado, las cantidades de átomos $H e^{3}$ y $H e^{4}$, están dadas por

$$
\hat{N}^{3}=\sum_{i}^{N}\left(1-s_{i}^{2}\right), \quad \hat{N}^{4}=\sum_{i}^{N} s_{i}^{2},
$$

y por lo tanto, el número total de átomos es $N=\hat{N}^{3}+\hat{N}^{4}$. De este modo, la concentración de átomos de $H e^{3}$ está dada por

$$
x=\frac{\hat{N}^{3}}{N}
$$

la cual representa un parámetro de orden adicional, que refleja la posibilidad de que ocurra una separación de fase. Para un sistema traslacionalmente invariable

$$
m=\left\langle s_{i}\right\rangle \quad \mathrm{y} \quad x=1-\left\langle s_{i}^{2}\right\rangle .
$$

En este caso, $\left\langle s_{i}\right\rangle$ y $\left\langle s_{i}^{2}\right\rangle$ representan la magnetización y el momento cuadrupolar medio, respectivamente, y tanto el ordenamiento superfluido como la separación de fase en la mezcla, se simulan mediante el ordenamiento magnético y cuadrupolar respectivamente.

Resumiendo, hasta acá se vio que el modelo presenta dos tipos de transiciones: fluidosuper fluido, y separación de fases, cuyos parámetros de orden son la Magnetización y la Concentración de $\mathrm{He}^{3}$, respectivamente.

El Hamiltoniano del modelo consiste en dos términos:

$$
H_{s}=-J \sum_{\langle i, j\rangle} s_{i} s_{j}
$$

donde $\langle i, j\rangle$ es la suma sobre los vecinos cercanos. Este término es el responsable de ordenamiento super fluido ya que lleva a una transición de segundo orden en la cual aparece un valor de $m$ distinto de cero para una temperatura crítica $T_{c}$. 
Cuando la concentración de $\mathrm{He}^{3}$ es cero, es decir, cuando todos los sitios están ocupados por átomos de $\mathrm{He}^{4}(1 \mathrm{y}-1)$, se recupera el modelo de Ising para un ferromagneto. Por otro lado, la presencia de $H e^{3}$ implica que cierto número de $s_{i}$ son cero, esto es similar a incluir impurezas no magnéticas en el sistema, provocando cierto "rechazo" a la transición y, por ende, la reducción de la $T_{c}$. Finalmente, cuando la concentración de átomos de $\mathrm{He}^{3}$ (B.3), es lo suficientemente grande, o dicho de otra manera, cuando la mayoría de los $s_{i}$ están ocupados por vacancias, la única forma que encuentra el sistema para "soportar" el orden super fluido, es separándose en dos fases, donde, la fase rica en $H e^{3}$ se mantiene en el estado normal (fluido). Esto es equivalente a un ordenamiento cuadrupolar en el modelo.

De esta manera, el modelo presenta dos tipos de ordenamiento: Magnético, el cual implica un orden super fluido, (un orden de los espines) y Cuadrupolar, el cual se da con vacancias, por ejemplo, cuando se genera un tablero de ajedrez espín-vacancia. Cuando esto ocurre, el super fluido pasa a ser fluido, por tal motivo, cuando hay muchas vacancias la única forma de que se mantenga el super fluido es que se separen las fases.

En este caso, la separación de las fases es una consecuencia directa del ordenamiento super fluido, pero al igual que en la mezcla de dos fluidos clásicos, este orden se puede inducir mediante las interacciones entre las componentes que no tengan comportamiento de super fluido. Por tal motivo, se introduce un nuevo término en el Hamiltoniano, el termino de interacción

$$
\begin{aligned}
H_{I}=-K_{33} \sum_{\langle i, j\rangle}\left(1-s_{i}^{2}\right)\left(1-s_{j}^{2}\right) & -K_{44} \sum_{\langle i, j\rangle} s_{i}^{2} s_{j}^{2}- \\
& -K_{34} \sum_{\langle i, j\rangle}\left[s_{j}^{2}\left(1-s_{i}^{2}\right)+s_{i}^{2}\left(1-s_{j}^{2}\right)\right],
\end{aligned}
$$

donde $-K_{\alpha \beta}$ representa la interacción $H e^{\alpha}-H e^{\beta}$. Desarrollando término por termino, el Hamiltoniano se puede escribir como: 
- Primer termino:

$$
\begin{aligned}
-K_{33} \sum_{\langle i, j\rangle}\left(1-s_{i}^{2}\right)\left(1-s_{j}^{2}\right) & =-K_{33} \sum_{\langle i, j\rangle} 1-s_{i}^{2}-s_{j}^{2}+s_{i}^{2} s_{j}^{2} \\
& =-K_{33}\left(\sum_{\langle i, j\rangle} 1-\sum_{\langle i, j\rangle} s_{i}^{2}-\sum_{\langle i, j\rangle} s_{j}^{2}+\sum_{\langle i, j\rangle} s_{i}^{2} s_{j}^{2}\right),
\end{aligned}
$$

- Tercer término

$$
\begin{aligned}
-K_{34} \sum_{\langle i, j\rangle}\left[s_{j}^{2}\left(1-s_{i}^{2}\right)+s_{i}^{2}\left(1-s_{j}^{2}\right)\right] & =-K_{34} \sum_{\langle i, j\rangle} s_{i}^{2}-s_{i}^{2} s_{j}^{2}+s_{j}^{2}-s_{i}^{2} s_{j}^{2} \\
& =-K_{34}\left(\sum_{\langle i, j\rangle} s_{i}^{2}-\sum_{\langle i, j\rangle} s_{i}^{2} s_{j}^{2}+\sum_{\langle i, j\rangle} s_{j}^{2}-\sum_{\langle i, j\rangle} s_{i}^{2} s_{j}^{2}\right),
\end{aligned}
$$

donde

$$
\sum_{\langle i, j\rangle} s_{i}^{2}=z \sum_{1}^{N} s_{i}^{2}, \quad \quad \sum_{i, j\rangle} s_{j}^{2}=z \sum_{1}^{N} s_{j}^{2}, \quad \sum_{\langle i, j\rangle} 1=z N,
$$

y $z$ y $N$ representan el número de vecinos cercanos y el número total de sitios de la red, respectivamente. Teniendo en cuenta esto

- Primer término

$$
-K_{33}\left(\sum_{\langle i, j\rangle} 1-\sum_{\langle i, j\rangle} s_{i}^{2}-\sum_{\langle i, j\rangle} s_{j}^{2}+\sum_{\langle i, j\rangle} s_{i}^{2} s_{j}^{2}\right)=-K_{33}\left(z N-2 z \sum_{1}^{N} s_{i}^{2}+\sum_{\langle i, j\rangle} s_{i}^{2} s_{j}^{2}\right),
$$

- Tercer término

$$
-K_{34}\left(\sum_{\langle i, j\rangle} s_{i}^{2}-\sum_{\langle i, j\rangle} s_{i}^{2} s_{j}^{2}+\sum_{\langle i, j\rangle} s_{j}^{2}-\sum_{\langle i, j\rangle} s_{i}^{2} s_{j}^{2}\right)=-K_{34}\left(2 z \sum_{1}^{N} s_{i}^{2}-2 \sum_{\langle i, j\rangle} s_{i}^{2} s_{j}^{2}\right),
$$

Uniendo todo

$$
\begin{aligned}
H_{I} & =-K_{33}\left(z N-2 z \sum_{1}^{N} s_{i}^{2}+\sum_{\langle i, j\rangle} s_{i}^{2} s_{j}^{2}\right)-K_{44} \sum_{\langle i, j\rangle} s_{i}^{2} s_{j}^{2}-K_{34}\left(2 z \sum_{1}^{N} s_{i}^{2}-2 \sum_{\langle i, j\rangle} s_{i}^{2} s_{j}^{2}\right) \\
& =-z N K_{33}+2 z K_{33} \sum_{1}^{N} s_{i}^{2}-K_{33} \sum_{\langle i, j\rangle} s_{i}^{2} s_{j}^{2}-K_{44} \sum_{\langle i, j\rangle} s_{i}^{2} s_{j}^{2}- \\
& -2 z K_{34} \sum_{1}^{N} s_{i}^{2}+2 K_{34} \sum_{\langle i, j\rangle} s_{i}^{2} s_{j}^{2} \\
& =\left(-K_{33}-K_{44}+2 K_{34}\right) \sum_{\langle i, j\rangle} s_{i}^{2} s_{j}^{2}+2 z\left(K_{33}-K_{34}\right) \sum_{1}^{N} s_{i}^{2}-z N K_{33} .
\end{aligned}
$$


Si bien existe la misma interacción interatómica entre todos los átomos de $\mathrm{He}$, en el líquido, la diferencia de masas y de estadísticas, llevan a interacciones efectivas diferentes entre átomos de $H e^{3}$ y $H e^{3}$. Esto se ve reflejado en las diferencias entre las $K_{\alpha \beta}$. Si todas fueran iguales, $H_{I}$ se reduciría a una constante, mientras que si fueran parecidas, la parte variable de $H_{I}$ sería una pequeña perturbación.

Por último, como $\left\langle\hat{N}^{3}\right\rangle$ y $\left\langle\hat{N}^{4}\right\rangle$ son cantidades conocidas, es posible introducir los potenciales químicos $\mu_{3}$ y $\mu_{4}$ para $H e^{3}$ y $H e^{4}$ respectivamente, e incorporarlos en el Hamiltoniano

$$
\mathscr{H}=H_{s}+H_{I}-\mu_{3} \hat{N}^{3}-\mu_{4} \hat{N}^{4}
$$

el cual se puede reescribir como

$$
\begin{aligned}
\mathscr{H}=-J \sum_{\langle i, j\rangle} s_{i} s_{j} & +\left(-K_{33}-K_{44}+2 K_{34}\right) \sum_{\langle i, j\rangle} s_{i}^{2} s_{j}^{2}+ \\
& +2 z\left(K_{33}-K_{34}\right) \sum_{1}^{N} s_{i}^{2}-z N K_{33}-\mu_{3} \hat{N}^{3}-\mu_{4} \hat{N}^{4}
\end{aligned}
$$

Teniendo en cuenta las ecuaciones (B.2), se puede escribir $\mathscr{H}$ como

$$
\begin{aligned}
\mathscr{H}=-J \sum_{\langle i, j\rangle} s_{i} s_{j} & +\left(-K_{33}-K_{44}+2 K_{34}\right) \sum_{\langle i, j\rangle} s_{i}^{2} s_{j}^{2}+ \\
& +\left[\mu_{3}-\mu_{4}+2 z\left(K_{33}-K_{34}\right)\right] \sum_{1}^{N} s_{i}^{2}-N\left(z K_{33}-\mu_{3}\right) .
\end{aligned}
$$

Finalmente, tomando

$$
\begin{array}{r}
\alpha=\left(K_{33}+K_{44}-2 K_{34}\right), \\
D=\left[\mu_{3}-\mu_{4}+2 z\left(K_{33}-K_{34}\right)\right],
\end{array}
$$

e ignorando la constante $N\left(z K_{33}-\mu_{3}\right)$, se puede escribir la versión final del Hamiltoniano del modelo de Blume-Emery-Griffiths

$$
\mathscr{H}_{B E G}=-J \sum_{\langle i, j\rangle} s_{i} s_{j}+D \sum_{i=1}^{N} s_{i}^{2}-\alpha J \sum_{\langle i, j\rangle} s_{i}^{2} s_{j}^{2},
$$





\section{BibLiOgRAFíA}

[1] J. Cardy, Scaling and Renormalization in Statistical Physics.

Cambridge Lecture Notes in Physics, Cambridge University Press, 1996.

[2] C. Domb, M. Green, and J. Lebowitz, Phase transitions and critical phenomena, vol. 8 of Phase Transitions and Critical Phenomena.

Academic Press, 1983.

[3] C. Alba-Simionesco, B. Coasne, G. Dosseh, G. Dudziak, K. Gubbins, R. Radhakrishnan, and M. Sliwinska-Bartkowiak, "Effects of confinement on freezing and melting," Journal of physics. Condensed matter : an Institute of Physics journal, vol. 18, p. 15—68, Feb 2006.

[4] A. O. Parry and R. Evans, "Influence of wetting on phase equilibria: A novel mechanism for critical-point shifts in films," Phys. Rev. Lett., vol. 64, pp. 439-442, Jan 1990.

[5] L. Boinovich and A. Emelyanenko, "Shift of triple point in confined systems with curved interfaces,” Molecular Physics, vol. 107, no. 17, pp. 1745-1753, 2009.

[6] M. Henkel, H. Hinrichsen, and S. Lübeck, Non-Equilibrium Phase Transitions. Volume 1: Absorbing Phase Transitions.

Springer Netherlands, 2008. 
[7] K. Binder, D. Landau, and M. Müller, "Monte carlo studies of wetting, interface localization and capillary condensation,” Journal of Statistical Physics, 2003.

[8] D. B. Abraham, "Solvable model with a roughening transition for a planar ising ferromagnet," Phys. Rev. Lett., vol. 44, pp. 1165-1168, May 1980.

[9] M. E. Fisher and H. Nakanishi, "Scaling theory for the criticality of fluids between plates," The Journal of Chemical Physics, vol. 75, no. 12, pp. 5857-5863, 1981.

[10] H. Nakanishi and M. E. Fisher, "Multicriticality of wetting, prewetting, and surface transitions," Phys. Rev. Lett., vol. 49, pp. 1565-1568, Nov 1982.

[11] H. Nakanishi and M. E. Fisher, "Critical point shifts in films," The Journal of Chemical Physics, vol. 78, no. 6, pp. 3279-3293, 1983.

[12] K. Binder, D. P. Landau, and D. M. Kroll, "Critical wetting with short-range forces: Is mean-field theory valid?," Phys. Rev. Lett., vol. 56, pp. 2272-2275, May 1986.

[13] K. Binder and D. P. Landau, "Wetting and layering in the nearest-neighbor simplecubic ising lattice: A monte carlo investigation," Phys. Rev. B, vol. 37, pp. 17451765, Feb 1988.

[14] K. Binder, D. P. Landau, and S. Wansleben, "Wetting transitions near the bulk critical point: Monte carlo simulations for the ising model," Phys. Rev. B, vol. 40, pp. 6971-6979, Oct 1989.

[15] E. Albano, K. Binder, D. W. Heermann, and W. Paul, "Adsorption on stepped surfaces: A monte carlo simulation,” Surface Science, vol. 223, no. 1, pp. 151 - 178, 1989.

[16] A. O. Parry, "Novel phase behaviour of a confined fluid or ising magnet," Physica A: Statistical Mechanics and its Applications, vol. 181, no. 3, pp. 250 - 296, 1992. 
[17] K. Binder, D. P. Landau, and A. M. Ferrenberg, "Character of the phase transition in thin ising films with competing walls," Phys. Rev. Lett., vol. 74, pp. 298-301, Jan 1995.

[18] K. Binder, D. P. Landau, and A. M. Ferrenberg, "Thin ising films with competing walls: A monte carlo study," Phys. Rev. E, vol. 51, pp. 2823-2838, Apr 1995.

[19] K. Binder, R. Evans, D. P. Landau, and A. M. Ferrenberg, "Interface localization transition in ising films with competing walls: Ginzburg criterion and crossover scaling," Phys. Rev. E, vol. 53, pp. 5023-5034, May 1996.

[20] A. Maciolek and J. Stecki, "d=2 ising strip with two surface fields solved using the transfer-matrix method,” Phys. Rev. B, vol. 54, pp. 1128-1144, Jul 1996.

[21] A. Maciolek, "Magnetization profiles for a ising strip with opposite surface fields," Journal of Physics A: Mathematical and General, vol. 29, pp. 3837-3845, Jul 1996.

[22] E. Carlon and A. Drzewiński, "Critical point shift in a fluid confined between opposing walls," Phys. Rev. E, vol. 57, pp. 2626-2633, Mar 1998.

[23] E. V. Albano, K. Binder, and W. Paul, "Monte carlo studies ofd= 2 ising strips with long-range boundary fields," Journal of Physics: Condensed Matter, vol. 12, pp. 2701-2723, Mar 2000.

[24] A. D. Virgiliis, E. Albano, M. Müller, and K. Binder, "Interfaces in the confined ising system with competing surface fields," Physica A: Statistical Mechanics and its Applications, vol. 352, no. 2, pp. 477 - 497, 2005.

[25] B. J. Schulz, K. Binder, and M. Müller, "First-order interface localizationdelocalization transition in thin ising films using wang-landau sampling,"Phys. Rev. E, vol. 71, p. 046705, Apr 2005. 
[26] L. Pang, D. P. Landau, and K. Binder, "Simulation evidence for nonlocal interface models: Two correlation lengths describe complete wetting," Phys. Rev. Lett., vol. 106, p. 236102, Jun 2011.

[27] E. V. Albano and K. Binder, "Wetting transition in the two-dimensional blume-capel model: A monte carlo study," Phys. Rev. E, vol. 85, p. 061601, Jun 2012.

[28] M. Blume, "Theory of the first-order magnetic phase change in uo 2 ," Phys. Rev., vol. 141, pp. 517-524, Jan 1966.

[29] H. Capel, "On the possibility of first-order phase transitions in ising systems of triplet ions with zero-field splitting," Physica, vol. 32, no. 5, pp. 966 - 988, 1966.

[30] M. Blume, V. J. Emery, and R. B. Griffiths, "Ising model for the $\lambda$ transition and phase separation in he ${ }^{3}-$ he $^{4}$ mixtures," Phys. Rev. A, vol. 4, pp. 1071-1077, Sep 1971.

[31] W. Selke, "Monte carlo studies of interfacial adsorption in multi-state models," Surface Science, vol. 144, no. 1, pp. 176 - 181, 1984.

[32] N. Fytas and W. Selke, "Wetting and interfacial adsorption in the blume-capel model on the square lattice," Eur. Phys. J. B, vol. 86, 2013.

[33] D. Mukamel and M. Blume, "Ising model for tricritical points in ternary mixtures," Phys. Rev. A, vol. 10, pp. 610-617, Aug 1974.

[34] D. Furman, S. Dattagupta, and R. B. Griffiths, "Global phase diagram for a threecomponent model," Phys. Rev. B, vol. 15, pp. 441-464, Jan 1977.

[35] M. Schick and W.-H. Shih, "Spin-1 model of a microemulsion,"Phys. Rev. B, vol. 34, pp. 1797-1801, Aug 1986. 
[36] K. E. Newman and J. D. Dow, "Zinc-blende-diamond order-disorder transition in metastable crystalline (GaAs) ${ }_{1-x}$ ge $_{2 x}$ alloys," Phys. Rev. B, vol. 27, pp. 74957508, Jun 1983.

[37] S. A. Kivelson, V. J. Emery, and H. Q. Lin, "Doped antiferromagnets in the weakhopping limit," Phys. Rev. B, vol. 42, pp. 6523-6530, Oct 1990.

[38] D. M. Saul, M. Wortis, and D. Stauffer, “Tricritical behavior of the blume-capel model," Phys. Rev. B, vol. 9, pp. 4964-4980, Jun 1974.

[39] Y.-L. Wang, F. Lee, and J. D. Kimel, "Phase diagrams of the spin-1 ising blume-emerygriffiths model: Monte carlo simulations," Phys. Rev. B, vol. 36, pp. 8945-8947, Dec 1987.

[40] A. N. Berker and M. Wortis, "Blume-emery-griffiths-potts model in two dimensions: Phase diagram and critical properties from a position-space renormalization group," Phys. Rev. B, vol. 14, pp. 4946-4963, Dec 1976.

[41] O. de Alcantara Bonfim and F. SáBarreto, "Renormalization group treatment of a spin-one ising model with biquadratic exchange interaction," Physics Letters A, vol. 109 , no. 7 , pp. $341-343,1985$.

[42] C. Buzano and L. Evangelista, "Cluster variation method for the blume-emerygriffiths model," Journal of Magnetism and Magnetic Materials, vol. 104-107, pp. $231-232,1992$.

[43] K. G. Chakraborty, "The reentrant behaviour of the spin-1 ising model in the effective-field approximation," Journal of Physics C: Solid State Physics, vol. 21, pp. 2911-2915, May 1988. 
[44] T. Kaneyoshi and E. Sarmento, "The application of the differential operator method to the blume-emery-griffiths model," Physica A: Statistical Mechanics and its Applications, vol. 152, no. 3, pp. $343-358,1988$.

[45] T. Kaneyoshi, "Correlated-effective-field treatment of spin-one ising models," Physica A: Statistical Mechanics and its Applications, vol. 164, no. 3, pp. 730 - 750, 1990.

[46] N. Metropolis, A. W. Rosenbluth, M. N. Rosenbluth, A. H. Teller, and E. Teller, "Equation of state calculations by fast computing machines," The Journal of Chemical Physics, vol. 21, no. 6, pp. 1087-1092, 1953.

[47] F. Wang and D. P. Landau, "Efficient, multiple-range random walk algorithm to calculate the density of states," Phys. Rev. Lett., vol. 86, pp. 2050-2053, Mar 2001.

[48] F. Wang and D. P. Landau, "Determining the density of states for classical statistical models: A random walk algorithm to produce a flat histogram," Phys. Rev. E, vol. 64, p. 056101, Oct 2001.

[49] R. E. Belardinelli and V. D. Pereyra, "Fast algorithm to calculate density of states," Phys. Rev. E, vol. 75, p. 046701, Apr 2007.

[50] D. Jasnow, "Critical phenomena at interfaces," Reports on Progress in Physics, vol. 47, pp. 1059-1132, Sep 1984.

[51] D. Bonn and D. Ross, "Wetting transitions," Reports on Progress in Physics, vol. 64, pp. 1085-1163, Aug 2001.

[52] D. Bonn, J. Eggers, J. Indekeu, J. Meunier, and E. Rolley, "Wetting and spreading," Rev. Mod. Phys., vol. 81, pp. 739-805, May 2009. 
[53] R. Brout, "Statistical mechanical theory of a random ferromagnetic system," Phys. Rev., vol. 115, pp. 824-835, Aug 1959.

[54] K. Binder and A. P. Young, "Spin glasses: Experimental facts, theoretical concepts, and open questions," Rev. Mod. Phys., vol. 58, pp. 801-976, Oct 1986.

[55] L. M. Luque, S. A. Grigera, and E. V. Albano, "Numerical simulations study of a spin-1 blume-emery-griffiths model on a square lattice," Journal of Statistical Mechanics: Theory and Experiment, vol. 2019, p. 033210, Mar 2019.

[56] E. V. Albano, L. Luque, M. L. Trobo, and K. Binder, "Numerical evidence of hyperscaling violation in wetting transitions of the random-bond ising model in $d=2$ dimensions," Phys. Rev. E, vol. 95, p. 022801, Feb 2017.

[57] M. E. J. Newman and G. T. Barkema, Monte Carlo methods in statistical physics. Oxford: Clarendon Press, 1999.

[58] P. Gagniuc, Markov Chains: From Theory to Implementation and Experimentation. John Wiley \& Sons, 2017.

[59] G. Torrie and J. Valleau, "Nonphysical sampling distributions in monte carlo freeenergy estimation: Umbrella sampling," Journal of Computational Physics, vol. 23, no. 2, pp. 187 - 199, 1977.

[60] B. A. Berg and T. Neuhaus, "Multicanonical ensemble: A new approach to simulate first-order phase transitions," Phys. Rev. Lett., vol. 68, pp. 9-12, Jan 1992.

[61] L. D. Landau and E. M. Lifshitz, Statistical Physics. London: MIT Press, 1966.

[62] M. E. Fisher, “The renormalization group in the theory of critical behavior," Rev. Mod. Phys., vol. 46, pp. 597-616, Oct 1974. 
[63] H. E. Stanley, "Scaling, universality, and renormalization: Three pillars of modern critical phenomena," Rev. Mod. Phys., vol. 71, pp. S358-S366, Mar 1999.

[64] M. Green, ed., Critical Phenomena, Academic Press, 1971.

[65] B. Widom, "Surface tension and molecular correlations near the critical point," The Journal of Chemical Physics, vol. 43, no. 11, pp. 3892-3897, 1965.

[66] A. D. Bruce, "Probability density functions for collective coordinates in ising-like systems," Journal of Physics C: Solid State Physics, vol. 14, pp. 3667-3688, Sep 1981.

[67] K. Binder, "Finite size scaling analysis of ising model block distribution functions," Zeitschrift für Physik B Condensed Matter, vol. 43, pp. 119-140, Jun 1981.

[68] N. Goldenfeld, Phase Transitions.

Westview Press, 1992.

[69] E. K. Riedel, "Scaling approach to tricritical phase transitions," Phys. Rev. Lett., vol. 28, pp. 675-678, Mar 1972.

[70] E. K. Riedel and F. J. Wegner, "Tricritical exponents and scaling fields," Phys. Rev. Lett., vol. 29, pp. 349-352, Aug 1972.

[71] Y. I. Dublenych, "Exact ground-state diagrams for the generalized blume-emerygriffiths model," Phys. Rev. B, vol. 71, p. 012411, Jan 2005.

[72] N. B. Wilding and A. D. Bruce, "Density fluctuations and field mixing in the critical fluid,” Journal of Physics: Condensed Matter, vol. 4, pp. 3087-3108, Mar 1992.

[73] J. Plascak and P. Martins, "Probability distribution function of the order parameter: Mixing fields and universality," Computer Physics Communications, vol. 184, no. 2 , pp. $259-269,2013$. 
[74] W. Kwak, J. Jeong, J. Lee, and D.-H. Kim, "First-order phase transition and tricritical scaling behavior of the blume-capel model: A wang-landau sampling approach,” Phys. Rev. E, vol. 92, p. 022134, Aug 2015.

[75] N. B. Wilding and P. Nielaba, "Tricritical universality in a two-dimensional spin fluid," Phys. Rev. E, vol. 53, pp. 926-934, Jan 1996.

[76] A. D. Bruce and N. B. Wilding, "Scaling fields and universality of the liquid-gas critical point," Phys. Rev. Lett., vol. 68, pp. 193-196, Jan 1992.

[77] M. S. S. Challa, D. P. Landau, and K. Binder, "Finite-size effects at temperaturedriven first-order transitions," Phys. Rev. B, vol. 34, pp. 1841-1852, Aug 1986.

[78] R. da Silva, N. A. Alves, and J. R. Drugowich de Felício, "Universality and scaling study of the critical behavior of the two-dimensional blume-capel model in short-time dynamics," Phys. Rev. E, vol. 66, p. 026130, Aug 2002.

[79] P. D. Beale, "Finite-size scaling study of the two-dimensional blume-capel model," Phys. Rev. B, vol. 33, pp. 1717-1720, Feb 1986.

[80] J. C. Xavier, F. C. Alcaraz, D. P. a. Lara, and J. A. Plascak, "Critical behavior of the spin- $\frac{3}{2}$ blume-capel model in two dimensions," Phys. Rev. B, vol. 57, pp. 1157511581, May 1998.

[81] C. J. Silva, A. A. Caparica, and J. A. Plascak, "Wang-landau monte carlo simulation of the blume-capel model," Phys. Rev. E, vol. 73, p. 036702, Mar 2006.

[82] H. J. W. Zandvliet, "The $2 d$ ising square lattice with nearest- and next-nearestneighbor interactions," Europhysics Letters (EPL), vol. 73, pp. 747-751, Mar 2006.

[83] S. Dietrich, "Critical phenomena at interfaces," Physica A: Statistical Mechanics and its Applications, vol. 168, no. 1, pp. 160 - 171, 1990. 
[84] M. L. Trobo, E. V. Albano, and K. Binder, "First-order and tricritical wetting transitions in the two-dimensional ising model caused by interfacial pinning at a defect line," Phys. Rev. E, vol. 90, p. 022406, Aug 2014.

[85] E. V. Albano and K. Binder, "Finite-size scaling approach for critical wetting: Rationalization in terms of a bulk transition with an order parameter exponent equal to zero," Phys. Rev. Lett., vol. 109, p. 036101, Jul 2012.

[86] D. P. Landau and K. Binder, A Guide to Monte Carlo Simulations in Statistical Physics.

Cambridge University Press, 3 ed., 2009.

[87] K. Binder, "Monte carlo study of entropy for face-centered cubic ising antiferromagnets," Zeitschrift für Physik B Condensed Matter, vol. 45, no. 1, pp. 61-69, 1981.

[88] L. Onsager, "Crystal statistics. i. a two-dimensional model with an order-disorder transition,” Phys. Rev., vol. 65, pp. 117-149, Feb 1944.

[89] R. Fisch, "Critical temperature for two-dimensional ising ferromagnets with quenched bond disorder," J Stat Phys, vol. 18, 1978.

[90] N. Fytas and P. Theodorakis, "Universality aspects of the $2 \mathrm{~d}$ random-bond ising and 3d blume-capel models," Eur. Phys. J. B, vol. 86, 2013.

[91] G. Forgacs, R. Lipowsky, and T. M. Nieuwenhuizen,Phase Transitions and Critical Phenomena, vol. 14 of Phase Transitions and Critical Phenomena. Academic Press, 1991.

[92] D. A. Huse and C. L. Henley, "Pinning and roughening of domain walls in ising systems due to random impurities," Phys. Rev. Lett., vol. 54, pp. 2708-2711, Jun 1985. 
[93] D. A. Huse, C. L. Henley, and D. S. Fisher, "Huse, henley, and fisher respond," Phys. Rev. Lett., vol. 55, pp. 2924-2924, Dec 1985.

[94] A. O. Parry, M. J. Greenall, and A. J. Wood, "Wedge covariance for two-dimensional filling and wetting," Journal of Physics: Condensed Matter, vol. 14, pp. 11691198, Jan 2002. 
